
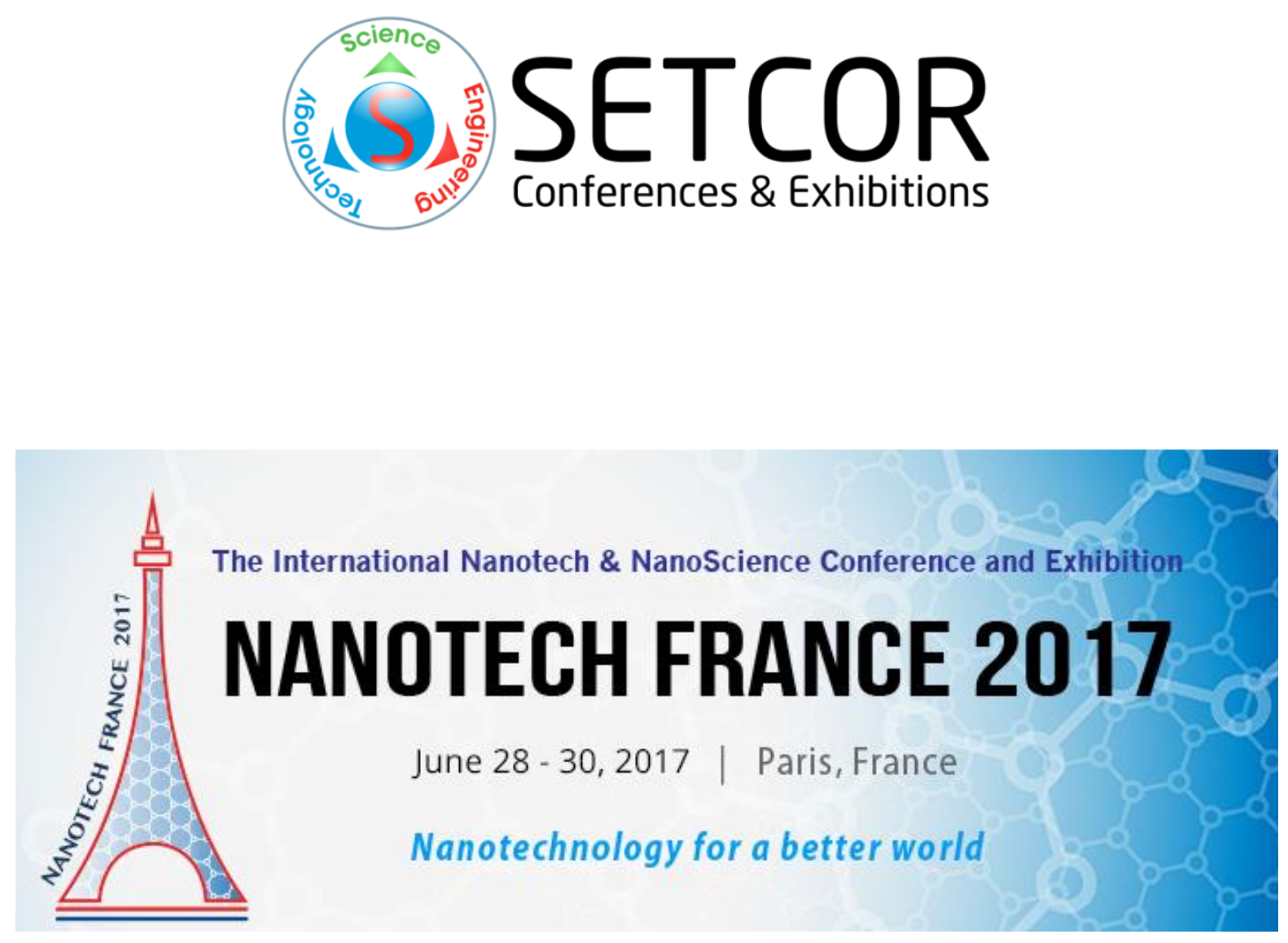

The International Conference for Nanotechnology \& Nanoscience

Nanotech France 2017

June 28 to 30, 2017, Paris - Korea

Conference Proceedings 


\title{
Polyurethane/Multi-Wall Carbon Nanotubes Composites for Microwave Applications
}

\author{
I. J. Rajmohan ${ }^{1}$, M. I. Hussein ${ }^{1}$, Y. Haik ${ }^{2}$, Q. Clément ${ }^{3 *}$ and N. Vukadinovic ${ }^{3}$ \\ ${ }^{1}$ United Arab Emirates University, Al-Ain, United Arab Emirates \\ ${ }^{2}$ Hamad Bin Khalifa University, Doha, Qatar \\ ${ }^{3}$ Dassault Aviation, 92552 Saint-Cloud, France \\ *Corresponding author: quentin.clement@ dassault-aviation.com
}

The increase of microwave sources has led to look for solutions to guarantee the protection of the devices with respect to the electromagnetic radiations. Absorbing materials have been developed in order to overcome this issue and thanks to the advance in material engineering, composite materials with a high microwave absorption efficiency have been progressively designed. Moreover, it has been shown that carbonaceous particles are promising candidates for designing high-performance microwave absorbers [1]. Among the various allotropic forms of carbon, the carbon nanotubes that correspond to a wrapped layer of carbon atoms organized in a hexagonal mesh leading to a cylindrical shape [2] have attracted considerable attention.

In this paper, we report on the electromagnetic properties of multi-wall carbon nanotubes (MWCNT) composites embedded in a polyurethane matrix and developed as lightweight materials for microwave applications. The considered CNT are either non functionalized (basic CNT) or coated (functionalized CNT) with cobalt or erbium nanoparticles. The frequency dispersive permittivity of composites is measured using an open-ended coaxial probe in the frequency range 0.1-13.6 GHz. From these experimental results, we show how these composites (including basic or functionalized CNT) can be used as efficient microwave absorbing materials through the reflection loss calculation.

The synthesis of the carbon nanotubes has been done by chemical vapor deposition (CVD) at the Hamad Bin Khalifa University. After purification, $95 \%$ of pure MWCNT have been synthesized. Their inner diameter is 5-12 nm, their outer diameter is $30-50 \mathrm{~nm}$ and their length is $10-20 \mu \mathrm{m}$. For functionalization, the CNT can then be coated by cobalt or erbium by adsorption of the magnetic nanoparticles on their external surface. This process is usually done with a very low nanoparticles content, so that the functionalized CNT composite doesn't have extra significant magnetic losses. Finally, the synthesis process of the composite consists in mixing the MWCNT into a polyurethane host matrix.

We synthesize several composites with different CNT concentrations (weight percentages) of $1 \mathrm{wt} \%, 2 \mathrm{wt} \%, 3 \mathrm{wt} \%, 4$ $\mathrm{wt} \%, 5 \mathrm{wt} \%, 8 \mathrm{wt} \%$ and $10 \mathrm{wt} \%$ for basic CNT composites. Functionalized CNT composites are also synthesized, with $1 \mathrm{wt} \% \mathrm{CNT}$ and $1 \mathrm{wt} \%$ for cobalt and erbium. An image of carbon nanotubes inside a composite material, taken with a transmission electronic microscope (TEM), is displayed in Figure 1, where they appear as long and thin wires mixed together.

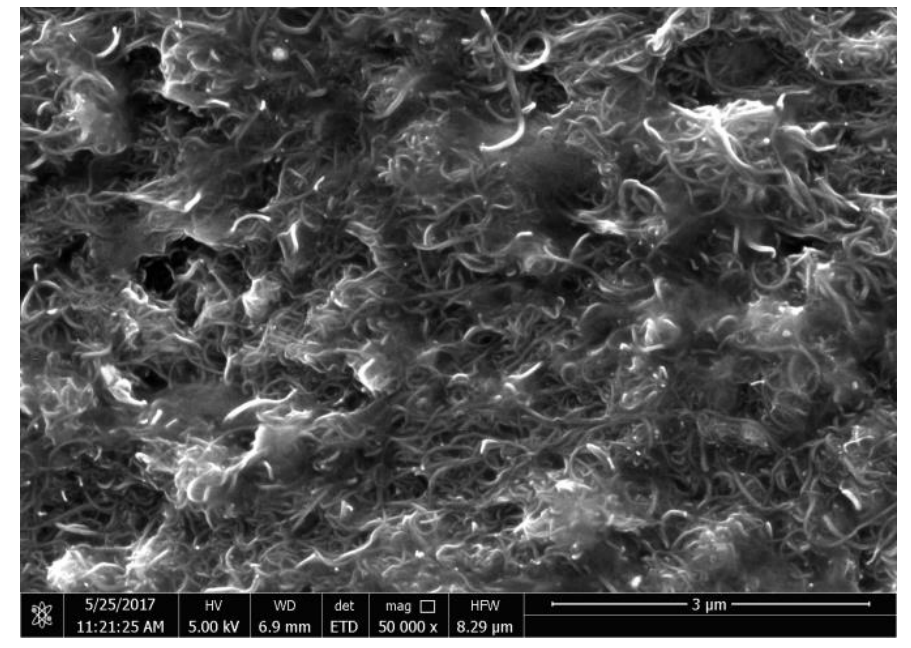

Figure 1. TEM image of a $10 \mathrm{wt} \%$ basic CNT composite.

The main parameter that needs to be known for electromagnetic applications is the permittivity of the composites. The permittivity measurement has been done at the United Arab Emirates University (UAEU) using an open-ended coaxial probe [3] in the frequency range 0.1-13.6 GHz. The $S_{11}$ parameter is measured with a probe connected to a Vector 
Network Analyzer (VNA). This parameter is a coefficient of the so-called S matrix, which describes the electromagnetic behavior of a material. Then, a numerical method enables to derive the complex permittivity from the $S_{11}$ measurement. We noticed, as expected, that the permittivity increases when the CNT concentration increases.

As the absorption efficiency of the material is linked to the dielectric loss tangent of the CNT composite, defined as $\tan (\square \square=\square, / \square$, functionalized CNT have been investigated to increase it, while keeping a reasonable CNT concentration. As displayed in Figure 2, the permittivity measurements of our functionalized $1 \mathrm{wt} \%$ CNT composites show the loss tangent increase, with a better efficiency for the cobalt-coated CNT composite.

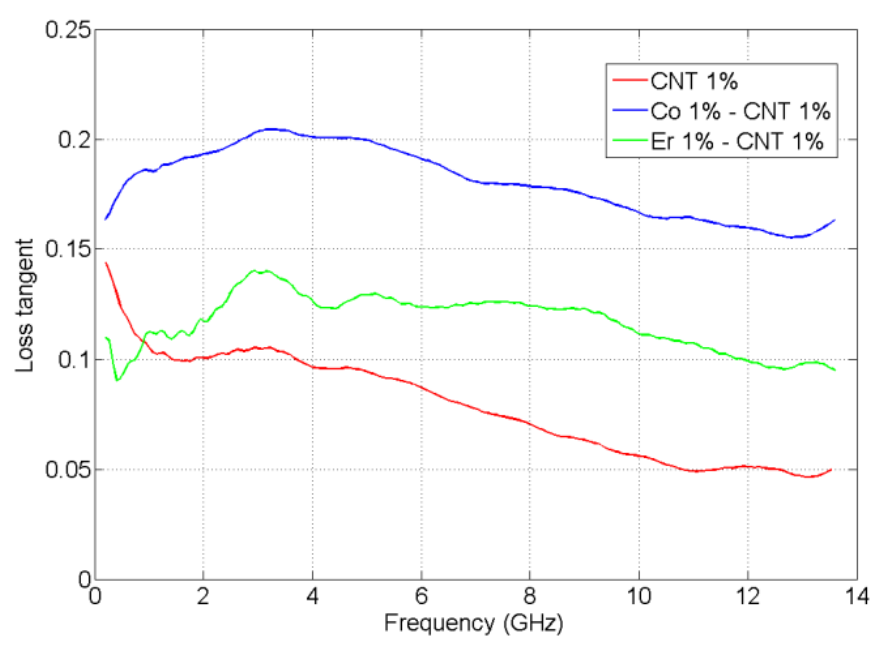

Figure 2. Comparison of the loss tangent of functionalized CNT composites with $1 \mathrm{wt} \%$ of cobalt, $1 \mathrm{wt} \%$ of erbium, and 1 wt $\%$ basic CNT composite.

The permittivity measurement enables to estimate the absorption efficiency of the CNT composites through the calculation of the reflection loss of a composite layer. The reflection loss calculation shows that the absorption spectrum is tuned when the CNT concentration changes or when the material thickness changes. Therefore, it is possible to strongly absorb the electromagnetic radiation in a spectral range of interest by a relevant design of the composite layer, that is to say by choosing the suitable CNT concentration and thickness. As an example, the reflection loss calculation at normal incidence of a 5 mm-thick composite layer for several basic CNT concentrations is shown in Figure 3, in the spectral range 0.1-14 $\mathrm{GHz}$.

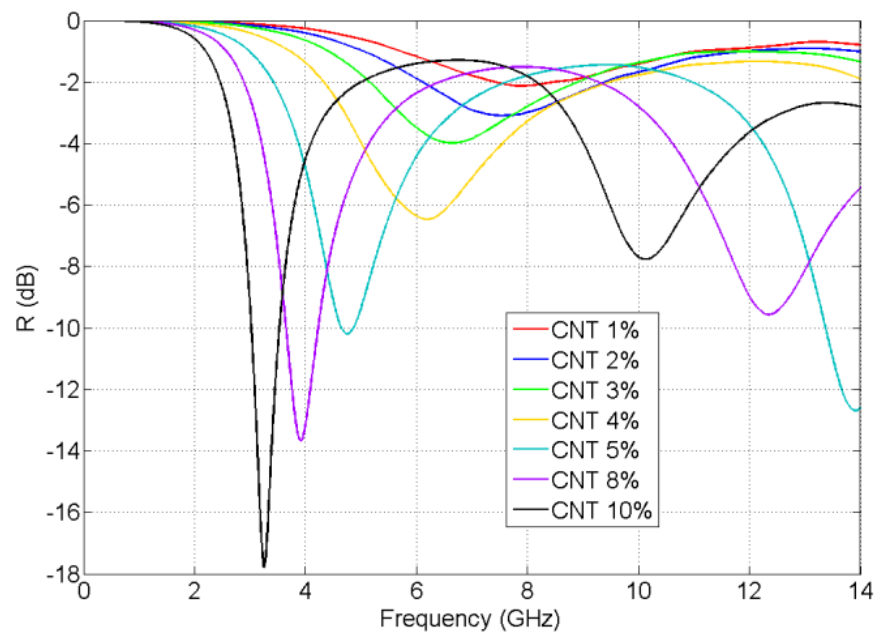

Figure 3. Reflection loss calculation of a 5 mm-thick composite layer at normal incidence, for different CNT concentrations.

The results show that an absorption level of $-10 \mathrm{~dB}$ can be reached in the 3-5 GHz range when the CNT concentration is above $5 \mathrm{wt} \%$. Therefore, this composite could be used as an efficient microwave absorbing material with a quite low CNT concentration and a light weight, mainly due to the low weight of the matrix material. Others minima of reflectivity can be found when the material thickness increases. Thus, we can design a single-band absorbing material with a low thickness or a multiple-band absorbing material with a higher thickness. 
The absorption efficiency of the functionalized CNT composites can then be compared to those of the basic CNT composites. The results are shown in Figure 4 and we see that the increase of the loss tangent enables to lower the reflectivity and so to enhance the absorption efficiency. As expected, the cobalt-coated CNT composite is more efficient than the erbium-coated one.

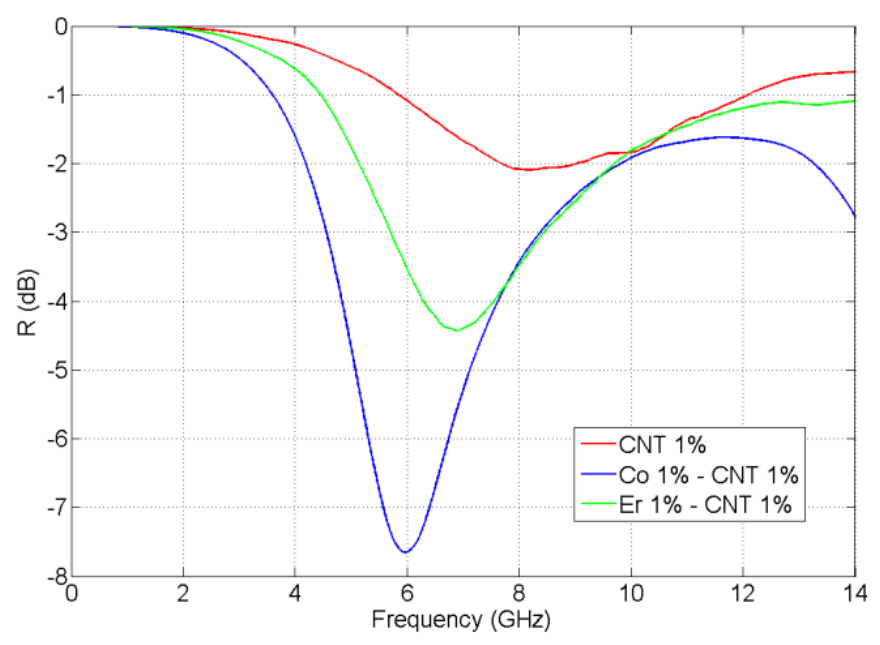

Figure 4. Comparison of the reflection loss at normal incidence of $5 \mathrm{~mm}$-thick $1 \mathrm{wt} \% \mathrm{CNT}$ composite layers, including cobalt-coated CNT, erbium-coated CNT and basic CNT.

Keywords: Carbon nanotubes, composites, polyurethane, microwave applications.

\section{Acknowledgement}

The authors gratefully acknowledge Dr. Ali H. Al-Marzouqi (Associate Dean for Research and Graduate Studies at College of Engineering, UAEU) and P. Piot (Research \& Cooperation Program Manager, Dassault Aviation) for the support on this project.

\section{References}

1. F. Qin and C. Brosseau, A review and analysis of microwave absorption in polymer composites filled with carbonaceous particles, J. Appl. Phys. 111 (2012) 061301.

2. S. Iijima, Helical microtubules of graphitic carbon, Nature 354 (1991) pp. 56-58.

3. T. P. Marsland and S. Evans, Dielectric measurements with an open-ended coaxial probe, IEE Proceedings HMicrowaves, Antennas and Propagation, (1987) pp. 341-349.

4. D. Y. K. Ko and J. R. Sambles, Scattering matrix method for propagation of radiation in stratified media: attenuated total reflection studies of liquid crystals, J. Opt. Soc. Am. A 5 (1988) pp.1863-1866. 


\title{
The Effect of Hybrid Nanofillers on Mechanical Properties of Carbon Fibre Epoxy Composites
}

\author{
A.A. Mahuof, G. Kotsikos A.G. Gibson, \\ School of Mechanical \& Systems Engineering, Newcastle University, Newcastle, UK
}

\begin{abstract}
:
In this study, graphene nanoplatelets (GNP) and cup-stacked carbon nanotubes (CSCNT) were used to enhance the interlaminar shear strength of carbon fibre epoxy composites. Ball milling was used to disperse these two types of nanofiller into the epoxy matrix at different weight concentrations $(0,2.5,4,5,6$, and 7 $\mathrm{wt} \%)$. Tensile and flexural strength measurements were used to identify the optimum weight concentration for each type of nano-additive. The highest mechanical strength in the nanocomposites was achieved with $5 \mathrm{wt} . \%$ of GNP, 6 wt.\% of CSCNT, and 4 wt.\% of a mixture of the two ingredients (2wt.\% each of GNP and CSCNT) in the epoxy matrix. The optimum weight concentration for each type of nanocomposite was then used to modify the mechanical properties of carbon fibre composite. Three types of carbon fibre epoxy composite hybrid were produced using hand lay-up (with GNP, CSCNT and an equal combination of the two). The results of short beam shear tests showed that the interlaminar shear strength increased by $29 \%$ with GNP, $34 \%$ with CSCNT and $32 \%$ with the hybrid mixture compared with conventional carbon fibre composite. TEM and SEMs were used to assess the dispersion of the nanofillers as well as the shear failure mechanism of the composite.
\end{abstract}

Keywords: Ball milling, hybrid nanofillers, carbon fibre composite, graphene, nanotubes.

\section{Introduction}

It is well-known that the use of a small proportion of certain nano-additives in the resin can significantly improve the strength and other properties of carbon or glass fibre composites (Thomas et al., 2014; Davis et al., 2010; Kim et al., 2011; Godara et al., 2010; Zhang et al., 2009; Gojny et al., 2005; Qian et al., 2010; Thostenson et al., 2005; Cai et al., 2003). In particular there has been interest in the use of single and multiwalled carbon nanotubes (Dassios et al., 2012; Yu and Files, 2000; Tjong, 2006; Gojny and Schulte, 2004; Gojny et al., 2005; Baur and Silverman, 2007, Dresselhaus, 2001; Bekyarova et al., 2007; Mahfuz et al., 2004; Shin et al., 2009). The well-known drawbacks of these nano-additives, which currently limit their use, are their high cost and the difficulty of dispersing them in the matrix due to their tendency to agglomerate (Yokozeki et al., 2012: Yu and Files, 2000; Ma et al., 2010). One possible route to reducing these problems could involve the use of mixtures of nano-additives (Al-Saleh, 2016; Kandare et al., 2015; Li et al., 2014).

In recent years, graphene nanoplatelets and cup-stacked carbon nanotubes have been considered as possible alternatives to conventional carbon nanotubes (Zhang et al., 2013; Wajid et al., 2013; Barrena et al., 2014; Landani, 2015; Yokozeki et al., 2009; Qin et al., 2015; Li et al., 2015).

The purpose of the present study is to investigate epoxy resins containing combinations of GNP and CSCNT and to use these in the manufacture of carbon fibre composites. The challenges lie in dispersing syntheses for both nanoparticles and achieving optimum dispersion without damage to the nano-additive. The ball milling technique was chosen as an effective practical technique to disperse the GNP and CSCNT epoxy resin. This technique generates high compressive and shear forces on the nanoparticles.

\section{Experimental work}

\subsection{Materials}

The resin used in this study was (RS-L135 epoxy, and RS-H137 hardener), supplied by the PRF composite material company. It was chosen because of its low viscosity and the ability to achieve a room temperature cure.

Nano-graphene GNP was supplied by XG Sciences, Inc. (Lansing, USA). The average platelet diameter was $1-5 \mu$, the nanothickness $(20 \mathrm{~nm})$, while the surface area was $\left(500 \mathrm{~m}^{2} / \mathrm{g}\right)$.

Cup-stacked carbon nanotubes (24PS HHT CSCNT) were supplied by CARBERE® (GSI Croes Corporation) in Japan. For this particle, the nanoscale diameter ranged from $70 \mathrm{~nm}$ to $80 \mathrm{~nm}$ and the length 
was $(5 \mu \mathrm{m})$. Two-dimensional carbon fibre cloth fabric (3k plain weave and $200 \mathrm{gsm}$ with a thickness of $0.3 \mathrm{~mm}$ ) was supplied by the Easy Composite Company in the UK.

\subsection{Optimisation of Nanocomposites}

\subsubsection{Preparation of Modified Epoxy Resin}

The weights of each component are shown in Table 1. In the preparation sequence, Figure 1, graphene nanoplatelets (GNP) and carbon nanotubes (CSCNT), as well as RS-L135 epoxy resin, were weighed and mixed together before being placed in the ball mill (FRITSCH, Germany) containing 350 balls of $5 \mathrm{~mm}$ diameter. The rotation speed of the closed container was $400 \mathrm{rpm}$ and the milling period was $45 \mathrm{~min}$. Subsequently, the RS-H137 hardener was added and blended by mechanical stirring for 2 min at 2,000 rpm. The resin was then vacuum de-gassed and poured into moulds. The specimens were cured for $48 \mathrm{hr}$ at $26^{\circ} \mathrm{C}$, followed by $15 \mathrm{hr}$ at $50^{\circ} \mathrm{C}$. The cured samples were removed from the mould for tensile and flexural measurements. The purpose of the two tests was to evaluate the optimum strength of three productions of epoxy nanocomposites matrix to use their modified matrix to enhance the mechanical properties of carbon fiber composite via the impregnation route using the hand lay-up method.

Table 1. Formulation of the epoxy nanocomposites.

\begin{tabular}{|c|c|c|}
\hline Sample & $\begin{array}{c}\text { GNP } \\
\text { Concentration wt. \% }\end{array}$ & $\begin{array}{c}\text { CSCNT } \\
\text { Concentration wt.\% }\end{array}$ \\
\hline Pure epoxy & 0 & 0 \\
\hline \multirow{4}{*}{$\begin{array}{c}\text { GNP/epoxy } \\
\text { nanocomposite }\end{array}$} & 2.5 & 0 \\
\cline { 2 - 3 } & 4 & 0 \\
\cline { 2 - 3 } & 5 & 0 \\
\cline { 2 - 3 } & 6 & 0 \\
\cline { 2 - 3 } & 7 & 0 \\
\cline { 2 - 3 } CSCNT/epoxy & 0 & 2.5 \\
\cline { 2 - 3 } nanocomposite & 0 & 4 \\
\cline { 2 - 3 } & 0 & 5 \\
\hline Hybrid & 0 & 7 \\
\cline { 2 - 3 } GNP- & 0 & 2 \\
\cline { 2 - 3 } CSCNT/epoxy & 1.25 & 2.5 \\
\cline { 2 - 3 } nanocomposite & 2 & 3 \\
\cline { 2 - 3 } & 2.5 & 3.5 \\
\cline { 2 - 3 } & 3 & 2 \\
\hline
\end{tabular}

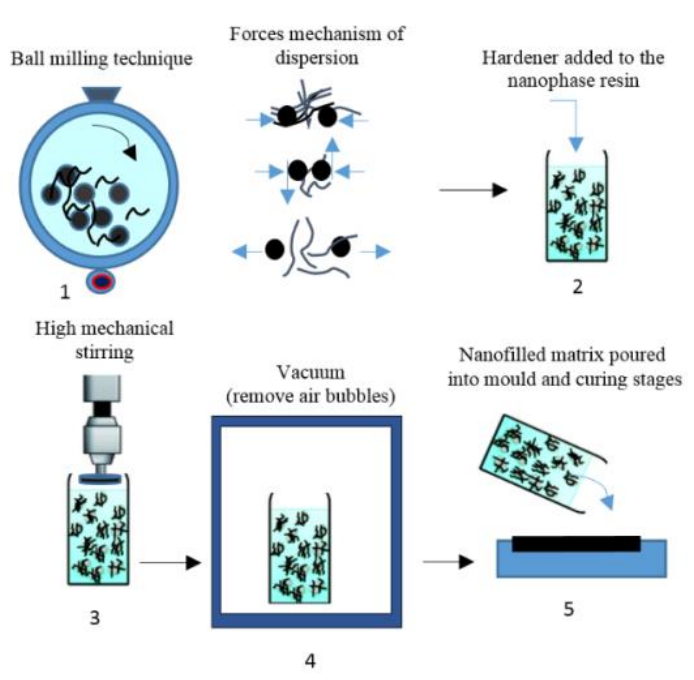

Fig.1: Nanofiller dispersion process in the epoxy resin. 


\subsubsection{Tensile Tests}

Tensile loading was applied on the three types of novel nanocomposites according to the ASTM D638 standard. A Shimadzu Universal Test Machine was used for this test at a cross head speed of $5 \mathrm{~mm} / \mathrm{min}$. The tests specimens were of "dogbone" with a gauge length of $25 \mathrm{~mm}$, a width of $6 \mathrm{~mm}$ and thickness of $4 \mathrm{~mm}$.

\subsubsection{Flexural Tests}

Three-point bend tests were conducted using a Shimadzu Universal Test Machine. The geometric dimensions of the specimens tested were length of $88 \mathrm{~mm}$, width $20 \mathrm{~mm}$ and a thickness of $5 \mathrm{~mm}$ (ASTM D790). All tests were performed at a constant cross head speed of $2 \mathrm{~mm} / \mathrm{min}$ and at room temperature.

\subsubsection{Microstructural Details}

The dispersion quality of nanofillers and fracture mechanism of the carbon fibre nanocomposites were assessed by using transmission electron microscopy (TEM) and scanning electron microscopy (SEMs). TEM was used to check the distribution of the nanoparticles and the mark of dispersion into the epoxy matrix. SEMs was carried out to provide images of the fracture surfaces of the tested samples in order to assess fracture mechanisms.

\subsection{Fabrication of Carbon Fibre/Epoxy Nanocomposite Hybrid}

The modified epoxy at optimum weight concentration was chosen to fabricate the design of the hybrid carbon fibre composite due to the highest improvement in strength properties. Hybrid laminated composites were fabricated by using woven fabric carbon fibre, 5 wt.\% GNP, 6 wt.\% CSCNT and 4 wt.\% GNP-CSCNT (hybrid nanofillers). The hand lay-up technique was used to fabricate four types of laminated composites modified and unmodified nanofillers, (i.e GNP/CF/epoxy nanocomposite, CSCNT/CF/epoxy nanocomposite, hybrid GNP-CSCNT/CF/epoxy nanocomposite and CF/epoxy composite unmodified as a guidance to check the enhancement in mechanical properties after adding the nanoparticles to the carbon fibre enhanced epoxy resin. The hybrid composites were manufactured utilising balanced symmetric stacking sequences of the woven carbon plies. Schematic diagrams of this fabricating synthesis are illustrated in Figure 2. However, the modified epoxy matrix was sufficiently impregnated into the woven fabric carbon. A laminate of peel ply was laid down on the flat glass mould to avoid adhesion of the laminated composites and to remove it easily after the curing stage. The layer of woven fabric was laid down on the peel ply. Next, the nanophase matrix was infused on both sides of each laminate uniformly by using suitable tools, such as a brush and roller. Subsequently, another sheet of peel ply was used to cover the upper surface of laminated composites. Two strips of steel plates were inserted as spacers to maintain the stability of pressure on the laminated composites and a homogeneous laminate thickness. The stacked laminates were then compressed using a steel plate inside the vacuum bagging, (Wicks et al., 2014), Nylon layer was used as an insulator to surround the steel plates. Then, a coat of breather cloth was laid down to absorb excessive resin and to secure a sufficient path for the vacuum pressure process. The vacuum bag film was laid down and plastered surround the laminated composites to insulate the composite from the influence of the environmental conditions. A valve connected to a vacuum bag was attached to the plastered part. The primary purpose of this vacuum pressure is to generate internal pressure force to improve the adhesion between the laminated composites and to remove the air completely from the vacuum bag. The neat weight concentration of the nanofilled matrix was estimated to be approximately $38 \%$ for each laminate composites. The nominal composite thickness was approximately $2 \mathrm{~mm}$. Two stages of thermal curing were performed for the panels of the hybrid composite as follows: the initial stage lasted for $48 \mathrm{hr}$ at $26^{\circ} \mathrm{C}$ under vacuum bagging pressure conditions, the laminated composites were then released from the vacuum bagging and followed by post curing for $15 \mathrm{hr}$ at $50^{\circ} \mathrm{C}$ as the secondary stage. The cured panel composite was machined and cut into suitable specimens with standard required dimensions to undertake the shear tests. 


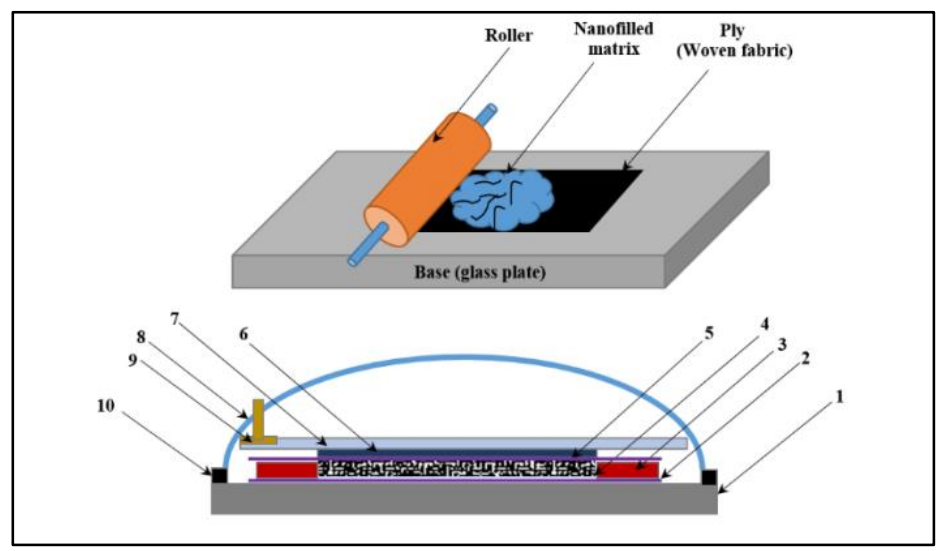

Fig.2: Manufacturing of hybrid carbon fibre/epoxy nanocomposite and experimental setup for vacuum curing: 1, glass plate (base); 2, peel ply; 3 , spacers; 4, carbon fibre fabric; 5 , peel ply; 6 , steel plate; 7 , breather materials; 8 , vacuum bag film; 9 , valve; 10 , sealant tape.

\subsection{Interlaminar Shear Strength (ILSS)}

Short beam specimens were prepared into standard dimensions with lengths of $20 \mathrm{~mm}$, widths of $10 \mathrm{~mm}$ and thickness of $2 \mathrm{~mm}$ (The European Standard EN ISO 14130: 1997). The tests were performed using the three point bend flexure test by Shimadzu Universal Test Machine; AG-X Plus at a cross head speed of $1 \mathrm{~mm} / \mathrm{min}$. Eight specimens at least were tested at room temperature. The maximum load was recorded and the interlaminar shear strength (ILSS) was calculated by applying the following equation 1 . The average value for each tested specimen type was recorded.

Interlaminar shear strength $($ ILSS $)=\left(3 \times \mathrm{F}_{\max } / 4 \times \mathrm{b} \times \mathrm{t}\right)$.

Where, $\mathrm{F}_{\max }$ is the maximum force and $\mathrm{b}$ and $\mathrm{t}$ are the width and the thickness of the sample, respectively.

\section{Results and discussion}

\subsection{Nanocomposite Morphology}

The TEM of the cross-sections of GNP/epoxy nanocomposite at 5wt.\%, CSCNT/epoxy nanocomposite at 6 wt. $\%$, and GNP-CSCNT/hybrid epoxy nanocomposite at 4wt.\% are shown in Figure 3. The images in Figure 3(a) and 3(b) show moderately good dispersion of and exfoliation of the nanofillers. However, some defects can also be seen. For example, some graphene agglomeration can be seen in Figure 3(a) and there is evidence of incomplete graphene exfoliation. Undispersed agglomerations of graphene and nanotubes can also be seen in Figure 3(c). In addition, the graphene agglomerates cause flaws in the hybrid matrix. The difficulty at dispersing graphene nanoplatelets with fibre nanotubes together into the epoxy resin is due to the reason that van der Waals forces in graphene network are probably stronger than that of fibre nanotube network. Figure 3(d) also reveals some nanotube breakage from the milling process. This was also reported by Konya et al., 2004.

Figure 4 shows SEMs of the fracture surfaces of the samples after testing. The images show some pull-out of the nanofillers, suggesting a toughening effect.

As a result of the fractography facts, other techniques and new methods, such as the combination of dispersion techniques maybe required and much better at dispersing the hybrid nanofillers uniformly (Liang et al., 2009). Therefore, the combination of sonication and ball milling techniques maybe preferred to improve the quality of dispersion and to achieve full exfoliation of the hybrid nanofillers in the epoxy resin (Guo \& Chen, 2014). Furthermore, the excellent dispersion induces synergy between the graphene and fibre nanotubes, which may cause improvements in the mechanical properties of the epoxy resin (Yue et al., 2014). 

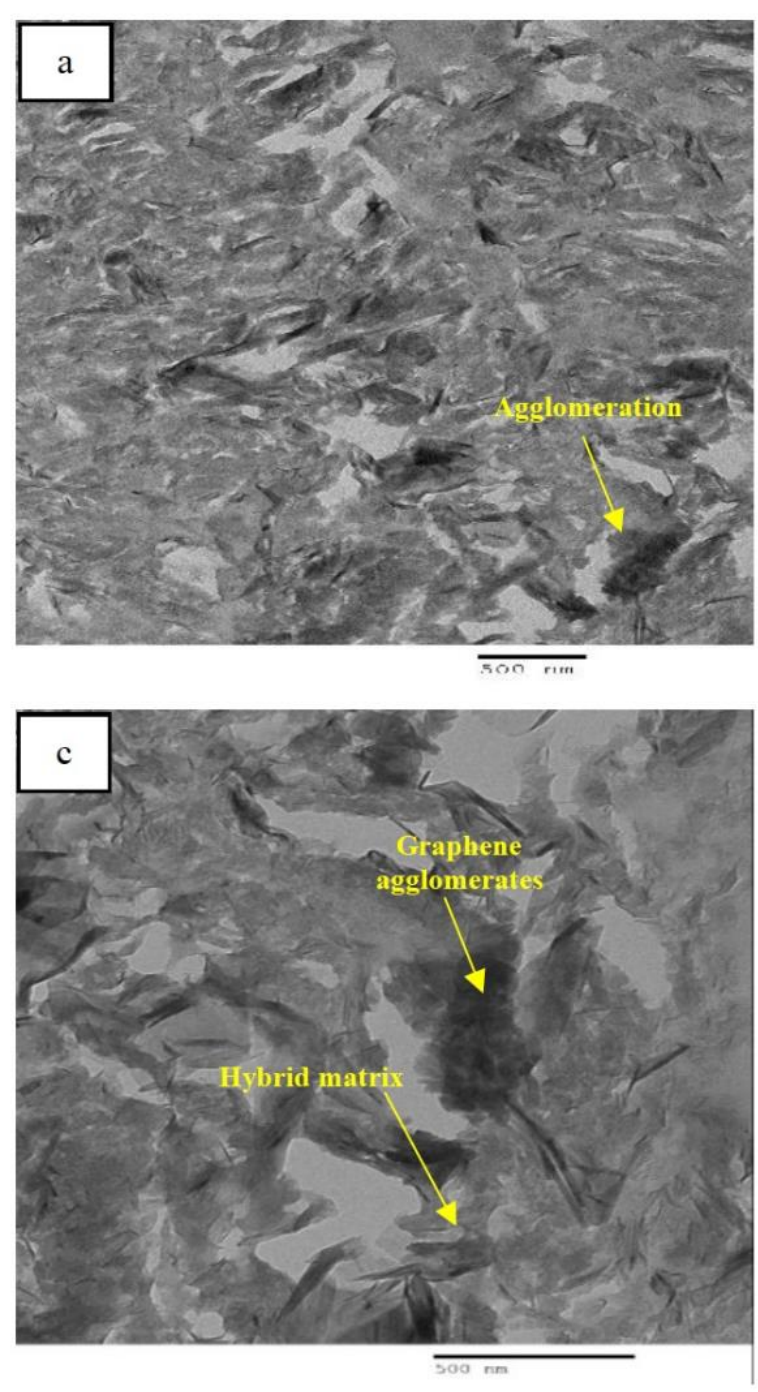
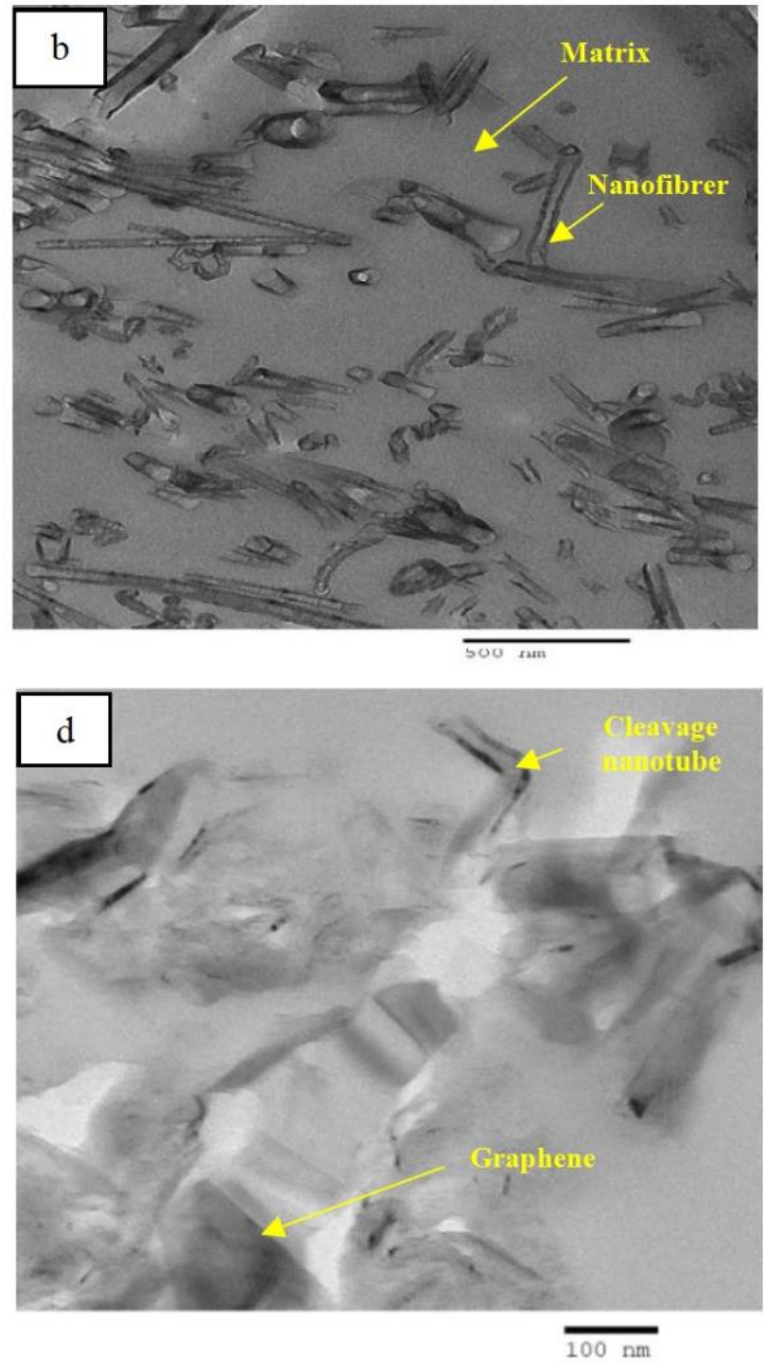

Fig.3: TEM images showing the dispersion for a) GNP/epoxy nanocomposite, b) CSCNT/epoxy nanocomposite, c) hybrid GNP-CSCNT/epoxy nanocomposite, d) breakage of a nanotube. 

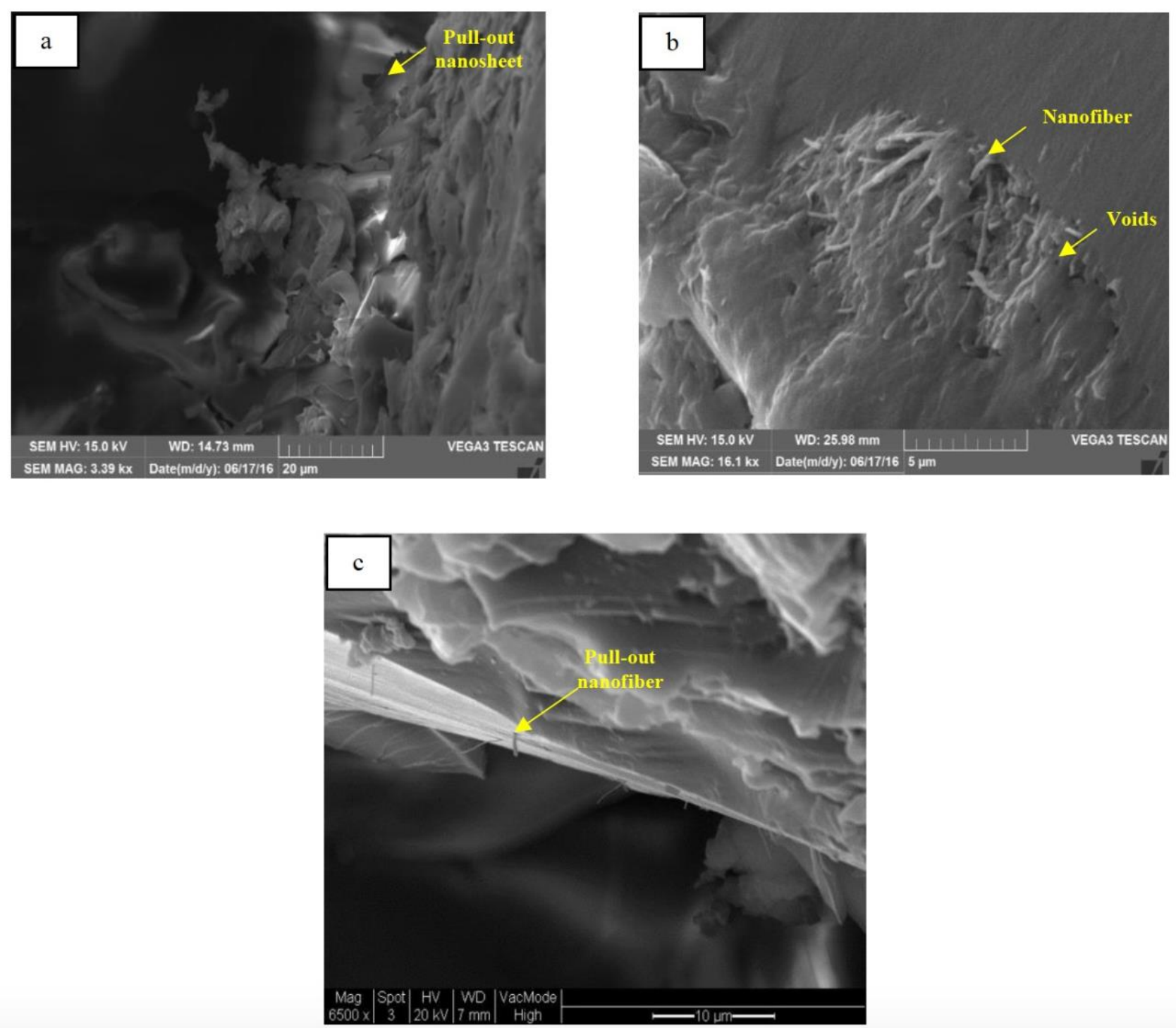

Fig.4: The SEMs images show the fracture mechanisms for three formulations of nanocomposites at critical weight concentrations: (a) GNP/epoxy nanocomposite, (b) CSCNT/epoxy nanocomposite, (c) hybrid GNPCSCNT/epoxy nanocomposite.

\subsection{Mechanical Properties of Nanocomposites}

\subsubsection{Tensile Response}

The tensile strength of GNP/epoxy nanocomposite, CSCNT/epoxy nanocomposite, and hybrid GNPCSCNT/epoxy nanocomposite increases with increasing GNP, CSCNT and GNP-CSCNT (hybrid nanofillers) contents as shown in Figure 5. A maximum improvement of approximately $17 \%$ in tensile strength was illustrated with the addition of 5wt.\% of GNP nanofiller, roughly 19\% with the addition CSCNT, and 10\% after the hybridisation of graphene nanoplatelets and fibre nanotubes into the epoxy resin. In addition, continuously increasing the tensile modulus of the hybrid nanofillers (GNP and CSCNT) by increasing the load level content, as shown in Figure 6. This behaviour illustrates that the hybridisation of the nanoparticles, such as graphene and fibre nanotube can have a positive effect on the tensile properties of the epoxy resin with level loads contents. However, Figure 6 also demonstrated that the tensile modulus of the modified epoxy gradually increases the fibre nanotube content, except for high load content (i.e. $7 \mathrm{wt}$. \%). The highest increment was recorded of approximately $18 \%$ at $6 \mathrm{wt}$. \%. The improvement in elastic modulus for the GNP nanocomposites fluctuated with loads level content. In contrast, the best improvement in elastic modulus was 
showed of about $12 \%$ after adding $5 \mathrm{wt}$. \% of GNP nanofiller. Moreover, the highest modulus of hybrid nanofillers was observed of approximately $14 \%$ at $6 \mathrm{wt} . \%$. In addition, there was a good improvement in this value at $4 \mathrm{wt} . \%$ by approximately $5 \%$.

The reasons for the increment in mechanical properties in relation to the three epoxy nanocomposites were perhaps due to pull-out and fractured nanoparticles, as shown in Figure 4.

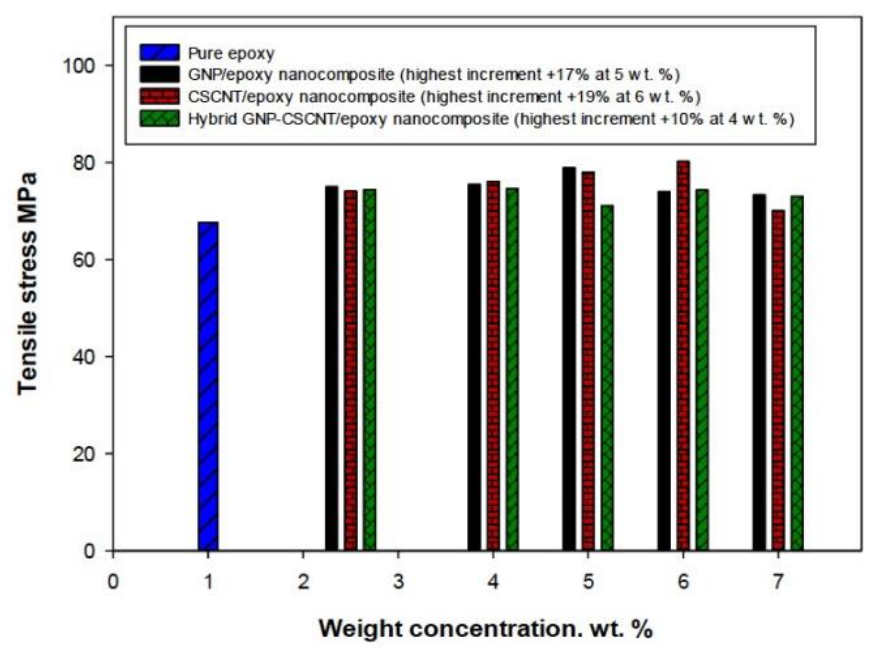

Fig.5: Comparison of tensile strength among three classes of modified matrix in nanocomposites.

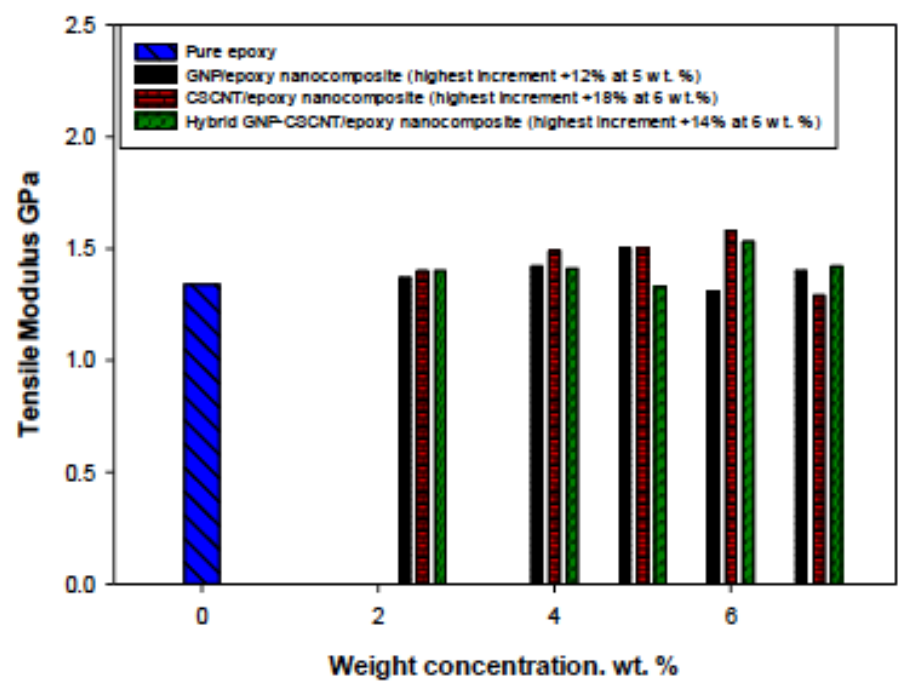

Fig.6: Comparison of tensile modulus among three classes of modified matrix in nanocomposites.

\subsubsection{Flexural Response}

The flexural properties of the modified matrix exhibit a continuous increment in flexural strength after adding graphene nanoplatelets and fibre nanotubes to the epoxy resin, in addition to the addition of hybrid nanofillers, as revealed in Figure 7. The highest improvement, $27 \%$ was recorded in the CSCNT/epoxy nanocomposite with the addition of $6 \mathrm{wt} . \%$ of the fibre nanotubes, $22 \%$ in the GNP/epoxy nanocomposite at $5 \mathrm{wt}$. \% and $17 \%$ after hybridisation $4 \mathrm{wt} . \%$ from graphene and fibre nanotube into the epoxy resin.

In contrast, the flexural modulus fluctuated in the three types of nanocomposites, as shown in Figure 8. The highest increment showed approximately $14 \%$ after adding $5 \mathrm{wt}$. $\%$ of the GNP, roughly $36 \%$ in the CSCNT/epoxy nanocomposite at $6 \mathrm{wt}$. $\%$ and $14 \%$ after the addition of $4 \mathrm{wt} . \%$ hybrid nanofillers. Generally, the highest increase in modulus pointed to a good dispersion and orientation of the nanofillers (Liu and Wu, 2001), and good adhesion between the surface area of the nanofiller and matrix (Zhao et al., 2010). Although there was an increase in flexural strength regarding the hybrid GNP-CSCNT/epoxy nanocomposites, the poor 
dispersion was evident in some regions of the matrix. In addition, the improvements in flexural strength properties of the hybrid nanocomposite were possibly due to the existence of some synergy between the graphene and fibre nanotube (Yue et al., 2014). The main reasons for variations in improving the strength properties of the hybrid nanocomposite maybe due to the damages in geometric structure of the nanoparticles (i.e. tortuosity or cleavage in nanotubes and agglutinative in graphene layers) during dispersion process ( $\mathrm{Li}$ et al., 2013). It is well known that maintaining the geometric structure of nanoparticles without damage is very necessary to reinforce the strength properties of the modified matrix (Yokozeki et al., 2008).

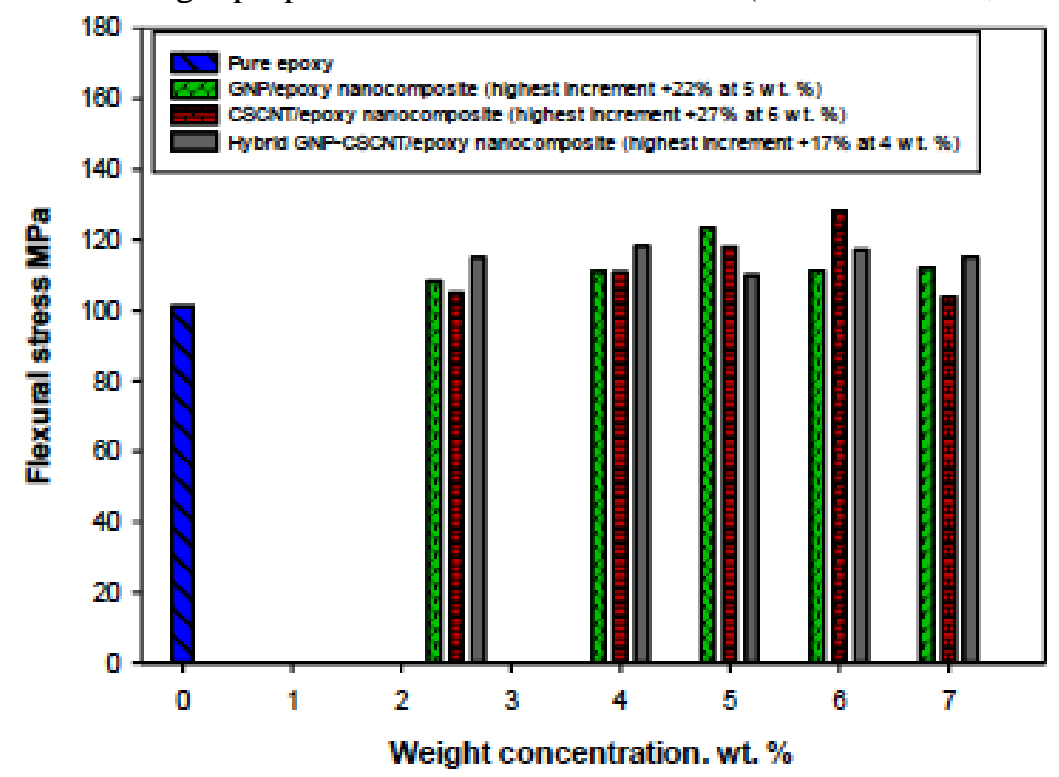

Fig.7: Comparison of flexural strength among three classes of modified matrix in nanocomposites.

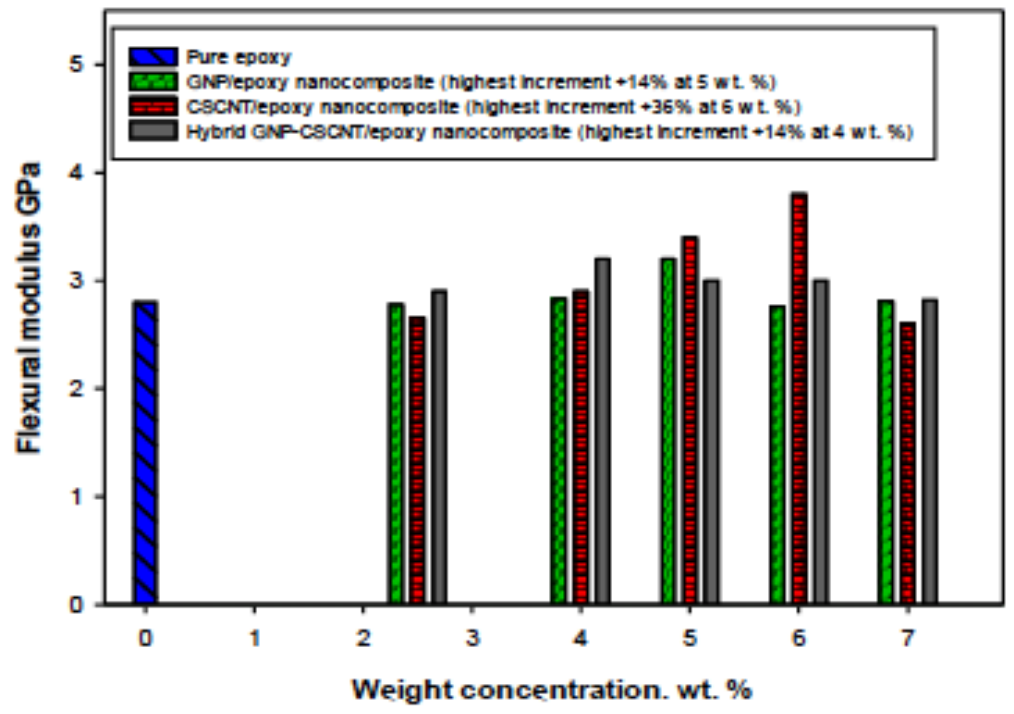

Fig.8: Comparison of flexural modulus among three classes of modified matrix in nanocomposites.

\subsection{Mechanical Properties of Carbon Fibre Nanocomposite Hybrid}

\subsubsection{Fracture Surface}

To explain the quality of the synthesised hybrid laminated composites. A cross-section of the fractured area views was assessed using SEMs. The mode of failure for most of the samples was multiple shear strength, as shown in Figure 9. In addition, the images in Figure 10 show the microstructural explanation of the hybrid composites (modified) and conventional carbon fibre epoxy composite (unmodified). The images reveal that massive cracks were evident in the matrix film at the top surface of tested samples after applying bending 
loading on the specimen of carbon fibre epoxy composite unmodified by nanofillers. The reason for these microstructural cracks is the low resistance of the unmodified carbon composite, smooth surface and the weak adhesion between the carbon fibre filaments and the epoxy matrix. Conversely, the lack these cracks in the modified matrix of hybrid composites indicates the high strength and toughness of the modified matrix, surface roughness of the nanofilled matrix, which increases the adhesion between the nanofilled matrix and carbon fibre network (Yokozeki et al., 2007).

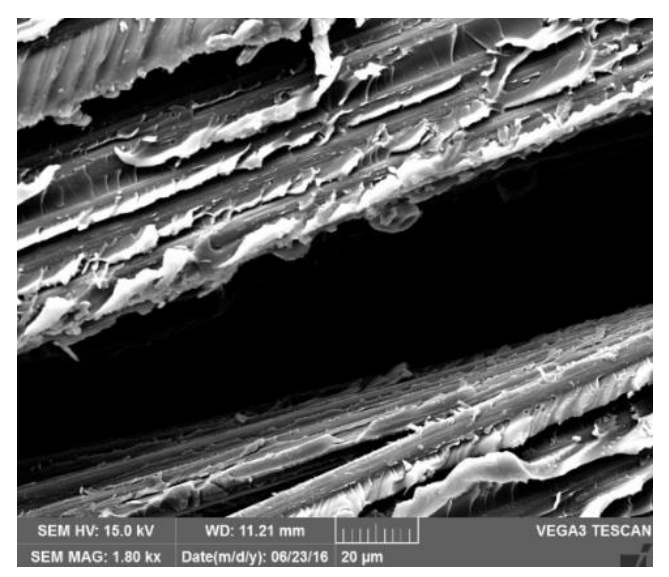

Fig.9: The SEMs image shows multiple shear failure regarding the hybrid carbon fibre composites modified by hybrid nanofillers.
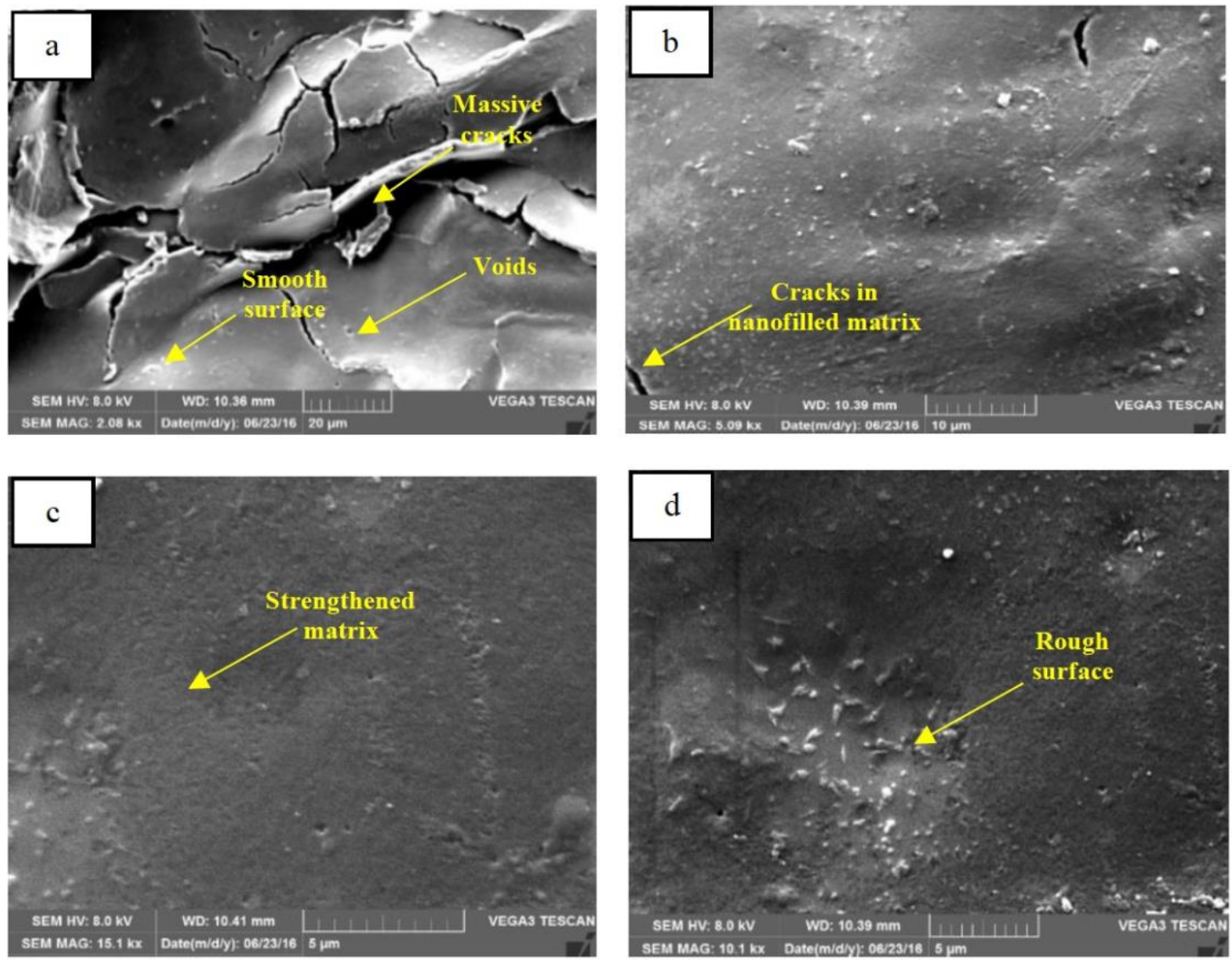

Fig.10: The SEMs images show the fracture surface of hybrid carbon fibre epoxy composite modified and unmodified composite at the top region of the tested samples: (a) CF/epoxy composite unmodified (b) 
GNP/CF/epoxy nanocomposite, (c) CSCNT/CF/epoxy nanocomposite, (d) hybrid GNP-CSCNT/CF/ epoxy nanocomposite.

\subsubsection{Shear Properties}

The stress-strain curves for each type of pure and hybrid composites are shown in Figure 13. The influence of the addition of the graphene, fibre nanotube and combined or hybrid nanofillers on the interlaminar shear force of the carbon fibre reinforced epoxy composites is shown in Figure 11. A comparison of the interlaminar shear strength (ILSS) of three types of hybrid carbon fibre epoxy composites is revealed in Figure 12. The experimental results demonstrate that there is an optimum weight content resulting in a maximum increase in shear strength of approximately 29\% in GNP/CF/epoxy nanocomposite, $34 \%$ in CSCNT/CF/epoxy nanocomposite, and $32 \%$ after impregnation of the hybrid nanofillers (GNP and CSCNT) onto the surface of carbon fibre network. The reasons for the increment in the interlaminar shear strength are probably due to the good strength properties of the modified matrix, which induce the adhesion between the fibre networks and the nanophase matrix. In addition, the hybridization of graphene nanoplatelets and fibre nanotubes enhances their synergy in the epoxy matrix which may cause increase the strength properties and toughness of the interface areas surrounding the fibre networks, (Kandare et al., 2015; Li et al., 2014; Zhang 2012; Zhu, 2007). Furthermore, the images in Figure 13 show the fracture mechanism of the carbon fibre modified with nanoparticles such as pull-out and fracture nanoparticles, which reflect the percentage of increment in the shear strength property (Fenner and Daniel, 2014).

As a result of these increments, the fibre nanotubes were observed to be a superior nanofiller in the enhancement of the mechanical properties of the carbon fibre composite.

In addition, the hybrid nanofillers exhibit a significant increment in shear strength properties of carbon fibre composite at the lowest load level content (i.e. 4 wt. \%). This indicates that the hybrid nanofillers have a positive effect on the mechanical properties of the carbon fibre composite.

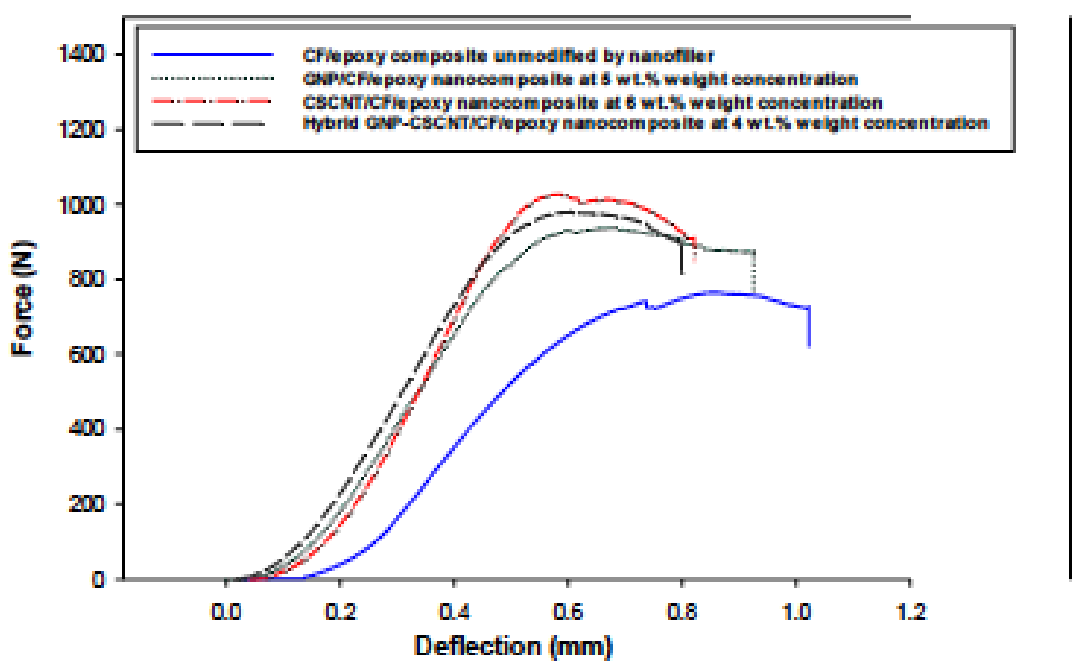

Fig.11: Typical force-deflection curves for short beam samples of carbon fibre epoxy composite modified by nanofillers at critical weight concentration. 


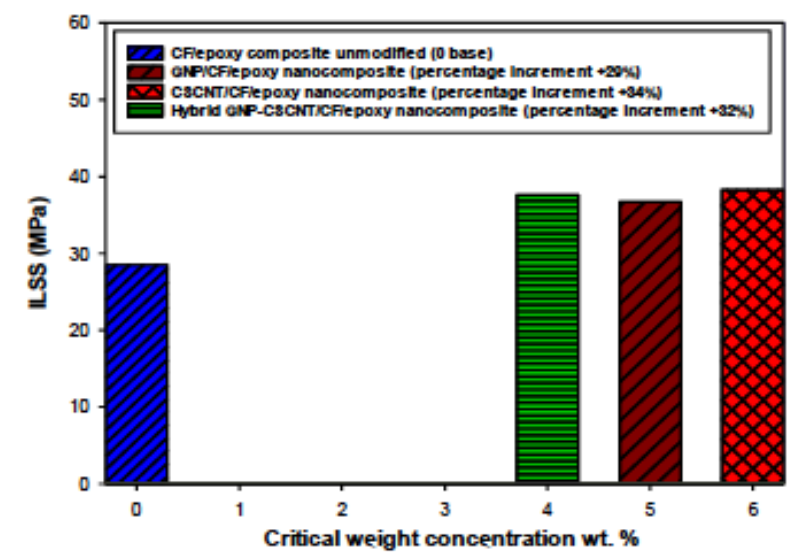

Fig.12: Comparison among three formulations of hybrid carbon fibre epoxy composite at optimum weight concentration.
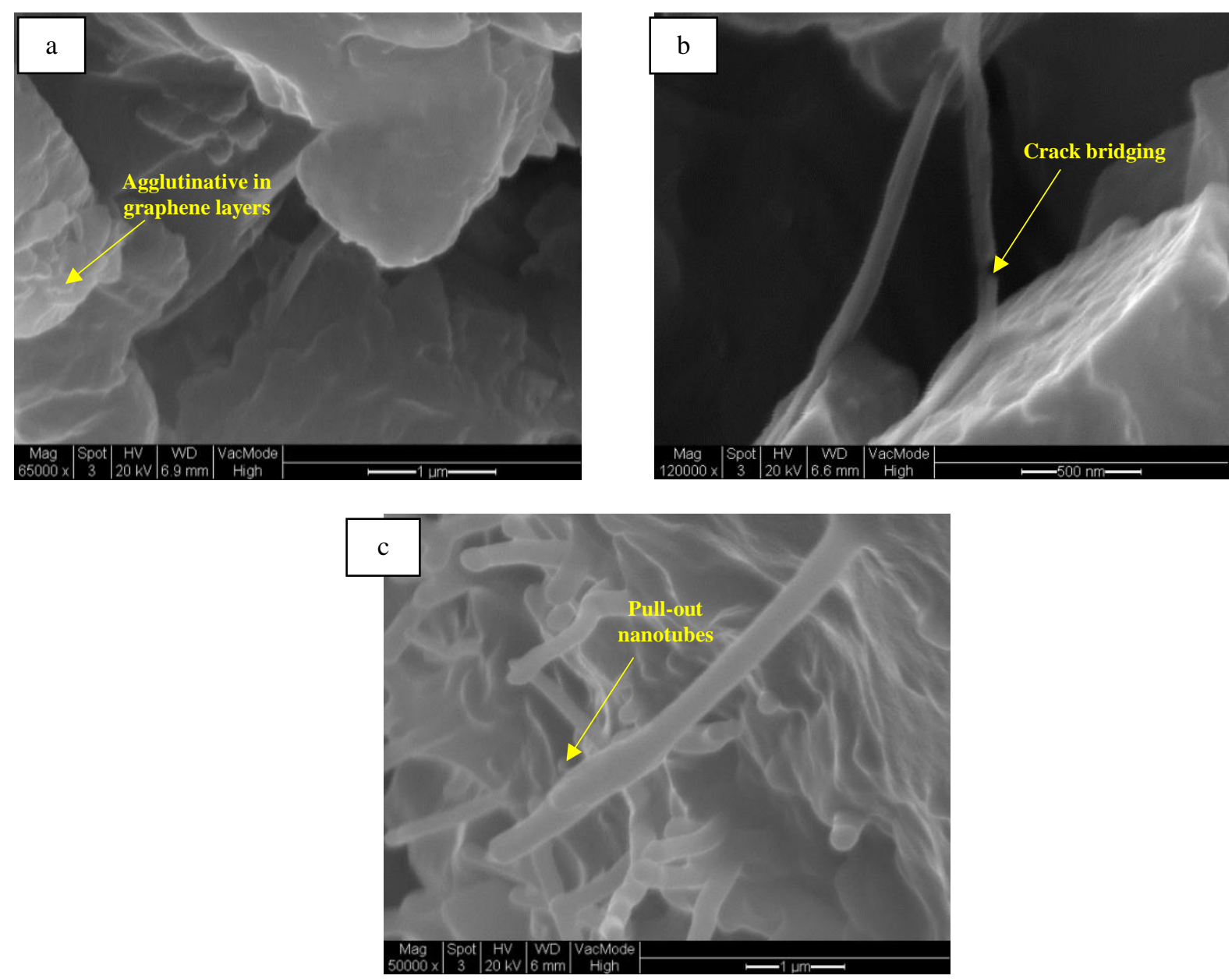

Fig.13: The SEMs images show the fracture mechanisms of three formulations of hybrid carbon fibre epoxy composite at the region failure: (a) GNP/CF/epoxy nanocomposite, (b) CSCNT/CF/epoxy nanocomposite, (c) hybrid GNP-CSCNT/CF/epoxy nanocomposite.

\section{Conclusion}

Three formulations of produced nanocomposites (i.e. GNP/epoxy nanocomposite, CSCNT/epoxy nanocomposites, and hybrid GNP-CSCNT/epoxy nanocomposite) were successfully prepared and dispersed via the ball milling technique. Three nanocomposites exhibit a significant influence on the strength properties 
of the epoxy resin. Hybrid nanocomposites containing graphene nanoplatelets and fibre nanotubes in the epoxy matrix have been promoted in this experimental study. Different mechanical properties investigated regarding the influence of two different nanofiller combinations. The tensile and flexural properties improved in the hybrid nanocomposites, the optimum strength properties was achieved after hybridisation of $4 \mathrm{wt}$. \% of graphene and fibre nanotubes into the epoxy resin, whereas, 5wt.\%, and $6 \mathrm{wt} . \%$ of non-hybrid nanocomposites. The effect of the hybridisation of graphene and fibre nanotubes on the interlaminar shear strength of carbon fibre reinforced epoxy composite is of great significance. An improvement of $32 \%$ was observed in this shear strength property after impregnation of the hybrid nanofilled matrix onto the surface of the carbon fibre network. As a consequence of these facts, hybridisation undoubtedly improved the strength properties of the epoxy resin and carbon fibre reinforced epoxy composites and contributed to somewhat decreasing the percentage of weight content of the nanofillers. Although there was a significant increment in the mechanical properties of the hybrid composites by applying the ball milling technique, several defects have been observed in the micro cross sections of the modified matrix via TEM and SEMs fractography concerning the dispersion mechanism, for instance, poor exfoliation of the hybrid nanofillers and some agglomerations. Thus, the combination of the dispersion techniques maybe necessary for modifying the exfoliation mark of nanoparticles into the epoxy resin.

\section{References}

1. Al-Saleh M H Electrical, "EMI shielding and tensile properties of PP/PE blends filled with GNP: CNT hybrid nanofiller", Synthetic Metal 217 (2016)322-330.

2. Barrena M.I, Salazar J.M, Soria A, Canas R., "Improved of the wear resistance of carbon nanofiber/epoxy nanocomposite by a surface functionalization of the reinforcement", Applied surface science289 (2014) 124-128.

3. Baur $\mathrm{J}$ and Silverman E., "Challenges and opportunities in multifunctional nano-composites structures for aerospace application”. MRS Bull 2007; 32:328-334.

4. Bekyarova E, Thostenson ET, Yu A, Kim H, Gao J, Tang J, et al. "Multiscale carbon nanotubecarbon fibre reinforcement for advanced epoxy composites". Langmuir 2007;23(7):3970-4.

5. Cai H., Yan F.Y., Xue Q.J., Lin W.M.., "Investigation of tribological properties of Al2O3-polyimide nanocomposites", Polym. Test. 22 (2003) 875-882.

6. Dassios KG, Musso S, Galiotis., "Compressive behaviour of MWCNT/epoxy composite mats". Comps Sci Technol. 1027-1033(2012)

7. Davis DC, Wilkerson JW, Zhu J, Ayewah DOO.“Improvements in mechanical properties of a carbon fiber epoxy composite using nanotube science and technology". Compos Sci Technol 2010;92(11):2653-62.

8. Dresselhaus MSDG., Avouris P.carbon nanotubes: synthesis, structure, properties and applications. Berlin:Springer-Verlag;2001.

9. Fenner J.S, Daniel I.M. "Hybrid nanorienforced carbon/epoxy composites for enhanced damage tolerance and fatigue life". Composites: Part A 65 (2014) 47-56.

10. Godara A, Gorbatikh L, Kalinka G, Warrier A, Rochez O, Mezzo L, et al. "Interfacial shear strength of a glass fiber/epoxy bonding in composites modified with carbon nanotubes". Compos Sci Technol 2010;70(9):1346-52.

11. Gojny FH, Schulte K. "Functionalization effect on the thermo-mechanical behaviour of multi-wall carbon nanotube/epoxy composites". Comps Sci Technol 2004;64(15);2303-8.

12. Gojny FH, Wichmann MHG, Fiedler B, Bauhofer W, Schulte K. "Influence of nano modification on the mechanical and electrical properties of conventional fibre reinforced composites". Composites Part A 2005;36:1525-35.

13. Gojny FH, Wichmann MHG, Fiedler B, Schulte K. "Influence of different carbon nanotubes on the mechanical properties of epoxy matrix composites - a comparative study". Compos Sci Technol 2005; 65:2300-2313

14. Guo W and Chen G. "Fabrication of Graphene/Epoxy Resin Composites with Much Enhanced Thermal Conductivity via Ball Milling Technique". J. APPL POLYM. SCI. 2014. DOI: 10.1002/APP. 40565. 
15. Kandare E, Khatibi A.A, Yoo S, Wang R, Ma J, Olivier P, Gleizes N, Wang C.H. "Improving the through-thickness thermal and electrical conductivity of carb fibre/epoxy laminates by exploiting synergy between graphene and silver nano-inclusions". Composites: Part A 69 (2015) 72-82.

16. Kim MT, Rhee KY, Lee JH, Hui D, Lau A. "Property enhancement of a carbon fiber/epoxy composite by using carbon nanotubes". Composites Part B 2011;42:1257-61.

17. Konya Z, Zhu J, Niesz K, Mehn D, Kiricsi I, "End morphology of ball milled carbon nanotubes". Carbon 42 (2004) 2001-2008.

18. Landani R.B, Wu S, Kinloch J.A, Ghorbani K, Zhang J, Mouitz A.P, Wang C.H,. "Improving the toughness and electrical conductivity of epoxy nanocomposites by using aligned carbon nanofibers"., Composites Science and Technology 117 (2015) 146-158.

19. Li W, Dichiara A, Bai J,. "Carbon nanotube-graphene nanoplatelet hybrid as high-performance multifunctional reinforcements in epoxy composites". Composites Science and Technology 74 (2013) 221-227.

20. Li W, Dichiara A, Zha J, Su Z, Bai J, "On the improvement of mechanical and thermo-mechanical properties of glass fabric/epoxy composites by incorporating $\mathrm{CNT}-\mathrm{Al}_{2} \mathrm{O}_{3}$ hybrids. ", Composite Science and Technology 103 (2014) 36-43.

21. Li Y, Zhao Y, Sun J, Hao Y, Zhang J, Han X, "Mechanical and Electromagnetic Interference Shielding Properties of Carbon Fiber/Graphene Nanosheets/Epoxy composite", Published online in Wiley Online Library (wileyonlinelibary.com), Society of Plastic Engineers, Polymer composites 2015 .

22. Liang Y, Ozawa M, Krueger A, "A general producer to fuctionlize agglomerating nanoparticles demonstrated on nanodiamond". ACSNANO318, 2288-2296(2009)

23. Liu $\mathrm{X}, \mathrm{Wu} \mathrm{Q}$,. "PP/clay nanocomposite prepared by grafting-melt interaction". Polymer 2001;42:10013-9.

24. Ma PC, Siddiqui N. A, Marom G, Kim J. K, "Dispersion and functionalization of carbon nanotubes for polymer-based nanocomposites": A Review, Composites: Part A 41 (2010) 1345-1367.

25. Mahfuz H, Adnan A, Rangari V.K, Jeelani S, Jang B.Z, "Carbon nanoparticles/whiskers reinforced composites and their tensile response". Compos. A:Appl. Sci. Manufact. 35 (2004) 519-527.

26. Qian, H., et al., "Carbon nanotube-based hierarchical composites: A review". Journal of materials chemistry, 2010. 20(23): p. 4751-4762.

27. Qin W, Vautard F, Drzal L.T, Yu J, "Mechanical and electrical properties of carbon fibre composites with incorporation of graphene nanoplatelets at the fibre-matrix interphase", Composites: Part B 69(2015) 335-341.

28. Shin J, Kim C, Geckeler K.E, "Single-walled carbon nanotube-polystyrene nanocomposites: dispersing nanotubes in organic media”.Polym.Int.58, 579-583 (2009).

29. Thomas S, Sinturel C, Thomas R., Micro-and nanostructured epoxy rubber blends., (2014).

30. Thostenson, E.T., C. Li, and T.-W. Chou, "Nanocomposites in context". Composites Science and Technology, 2005. 65(3-4): p. 491-516.

31. Tjong SC. "Structural and mechanical properties of polymer nanocomposites". Mater Sci Eng R 2006; 53: 73-197.

32. Wajid A, Tanvir A. H. S, Das Sriya, Irin Fahmida, Jankowski Alan F, Green Micah J, "HighPerformance Pristine Graphene/Epoxy Composites With Enhanced Mechanical and Electrical Properties". Macromol. Mater. Eng. 298 (2013) 339-347.

33. Wicks S.S, Wang W, Williams M.R, Wardle B.L, "Multi-Scale interlaminar fracture mechanisms in woven composite laminates reinforced with aligned carbon nanotubes". Composites Science and Technology 100 (2014) 128-135.

34. Yokozeke T, Iwahori Y, Ishiwata S,. "Matrix cracking behaviours in carbon fibre/epoxy laminates filled with cup-stacked carbon nanotubes $\left(\mathrm{CSCNT}_{\mathrm{s}}\right)$ ". Composites: Part A 38(2007) 917-924.

35. Yokozeki T, Aoki T, Arai A, Ishilbashi M, Ynagaisawa T, Imai K, Takahashi T. "Effect of CNT length distribution on mechanical properties of nanocomposites: measurement and estimation". Proceeding of 13th US-Japan Conference on Composite material 2008, CD-ROM. 
36. Yokozeki T, Iwahori Y, Ishibashi M, Yanagisawa Takashi, Imai K, et al., Fracture toughness improvement of CFRP laminates by dispersion of cup-stacked carbon nanotubes". Composites Science and Technology 69 (2009) 2268-2273

37. Yokozeki T, Schulz S, Buschhorn S, Schult K. "Investigation of shear thining behavior and microstructure of MWCNT/epoxy and CNF/epoxy suspensions under steady shear conditions". European Polymer Journal (2012); 48: 1042-1049.

38. Yu MF, Files BS, Arepalli S, Ruoff RS. " Tensile loading of ropes of single wall carbon nanotubes and their mechanical properties". Phys Rev Lett 2000;84(24):5552-5.

39. Yue L, Pircheraghi G, Monemain S.A, Zloczower I.M, “ Epoxy composites with carbon nanotubes and graphene nanoplatelets-Dispersion and synergy effects". Carbon 78 (2014) 268-278.

40. Zhang F, Wang R, He X, Wang C. "Interfacial shearing strength and reinforcing mechanisms of an epoxy composite reinforced using a carbon nanotube/ carbon fiber hybrid". J Mater Sci 2009;44:3574-7.

41. Zhang Wen, Yi Min, Shen Zhigang, Zhao Xiaohu, Zhang Xiaojing, Ma Shulin., "Graphenereinforced epoxy resin with enhanced atomic oxygen erosion resistance", J. Mater. Sci. 48 (2013), 2416-2423.

42. Zhang X, Fan X, Yan C, Li H, Zhu Y, Li X, Yu L., "Interfacial microstructure and properties of carbon fiber composites modified with graphene oxide"., ACS Appl. Mater. Interfaces 2012, 4, 1543-1552.

43. Zhao X, Zhang Q, Chen D, Lu P., "Enhanced mechanical properties of graphene-based poly (vinyl alcohol) composites". Macromolecules 2010, 43, 2357-2363.

44. Zhu J, Imam A, Crare R, Lozano K, Khabashesku V.N, Barrera E.V. Compos Sci. Technol.2007, 67, 1504-1517. 


\title{
Implementation of a new computational strategy in the design of more effective molecularly imprinted polymers
}

\author{
Ghada AlTaher ${ }^{1}$, Tarek M. Madkour ${ }^{2}$ \\ ${ }^{1}$ American University in Cairo, Nanotechnology graduate program, Cairo, Egypt \\ ${ }^{2}$ American University in Cairo, Department of chemistry, Cairo, Egypt
}

\begin{abstract}
In this paper, a novel feature (Conformational stability) is presented that is to the best of our knowledge has not been studied before for MIPs. The paper adopted initially a theoretical approach followed by experimental investigations and characterizations. In the theoretical approach, a virtual library of thirty monomers was created. The monomers were polymerized to create thirty polymeric ensembles. The conformational stability of the ensembles was assessed by selecting a $T_{B}$ (Boltzmann parameter) value for each polymeric ensemble at which the polymer altered its conformation. Moreover, cohesive energies (CE) for each monomer were calculated by molecular dynamics MD. The $T_{B}$ and $C E$ values were used to screen the thirty monomers on two stages and select the best promising two candidates. The selected candidates were experimentally employed in MIP synthesis. The resulting MIPs (MIP2 and MIP3) could show high performances and exhibited exceptionally high imprinting factors IF compared to that of control MIP1.
\end{abstract}

Keywords: MIPs, computational modeling, molecular dynamics, nano-scaled cavities, binding energy, conformational analysis.

\section{Introduction}

In the last few decades, Molecularly Imprinted Polymers (MIPs) emerged as potential substitutes to enzymes/proteins in chemical sensing applications [1]. They are polymer based and thus, more stable and much cheaper.

MIP networks contain nano-scaled cavities that could bind complimentary analytes during chemical sensing. However MIPs are not as highly specific as enzymes/proteins, owing to the deterioration of many cavities during the synthesis stages [2,3]. The Literature usually focused on creating binding sites that can bind strongly with the template [4,5]. It has been manifested that this is not always enough!.

This research adopted a novel strategy in order to induce the formation of nano-scaled cavities that not only could bind strongly to a particular template, but also resisted deterioration.

\section{Methodology}

\subsection{Theoretical investigations}

Stage A:Primary screening

Thirty monomers were analyzed by Conformational analysis \& MD.

Stage B:

Four monomers were selected for further secondary analysis, based on their high conformational rigidity and cohesive energy scores CE.

Stage C:Secondary screening

The four selected monomers underwent extensive additional secondary analysis, by the same computational techniques (Conformational analysis \& MD).

\subsection{Experimental investigations}

From stage $\mathrm{C}$, the best two scoring monomeric candidates were selected and employed in the synthesis of the MIPs (MIP2 \& MIP3). A control MIP (MIP1) was also synthesized using a low scoring monomer.

Template rebinding experiments (towards glucose) for both the MIPs and their respective Non Imprinted Polymers (NIPs) were conducted. 


\section{Results and Discussion}

\subsection{Theoretical investigations}

\section{Stage A}

According to Arrhenius equation

$\mathrm{T} \boldsymbol{m}=\boldsymbol{A} \boldsymbol{e}^{\frac{E a}{\boldsymbol{R} T}}$, the orientation time $\mathrm{T} \boldsymbol{m}$ which reflects the ease of uncoiling of polymeric chains is proportional to $\mathrm{T}_{\mathrm{B}}$ (Boltzmann parameter) [6]. As the $\mathrm{T}_{\mathrm{B}}$ value at which the polymeric ensembles altered their conformations increased, such polymeric chains could strongly resist conformational changes, and consequently are expected to produce more rigid stable nano-scaled cavities within their respective MIP networks.

Stage B:

The following approach was adopted in order to select the best performing 4 monomeric candidates with respect to their high $T_{B}$ and $C E$ values Fig. 1.

Stage C:

Further extensive secondary analyses led to the selection of; AMPSA, BHPEA, and AA (control) for the experimental investigations Table 1.

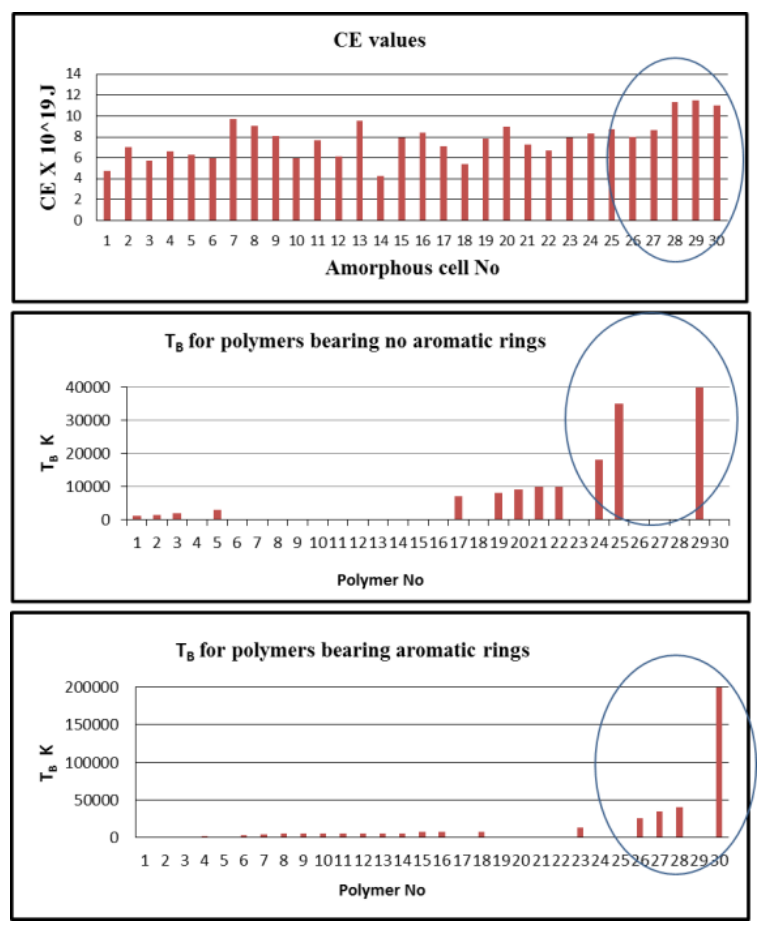

Fig. 1 The strategy of selection of the four monomers for secondary analysis. The oval rings show the selected polymers for further secondary analyses.

Table 1 shows the selected monomers for experimental investigations.

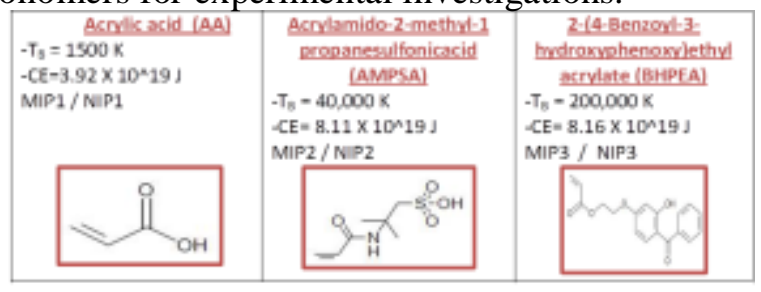

\subsection{Experimental investigations} Template rebinding experiments: 
Fig. 2 shows the amount of glucose bound $C_{b}$ by each polymer. Only the MIPs could successfully bind to the template significantly. Thus MIPs were successfully imprinted by the template and could recall its recognition memory.

Although MIP2 binding capacity significantly exceeded that of MIP3, but still the reproducibility of the binding capacity results within MIP3 was better than MIP2. This could be attributed to the exceptionally high conformational stability of BHPEA polymeric chains (TB=200,000 K).

\section{Scanning Electron Microscopy (SEM)}

Fig. 3 shows SEM micrographs of MIP 1 and NIP 1. The porosity distributions in MIP1 mimic that of NIP1 which correlates directly with their insignificant binding performance.

However, the template presence during MIP synthesis has homogenized the distribution of pores and pore channels within MIP2 and MIP3 compared to their respective NIPs Fig. 4 and Fig. 5.

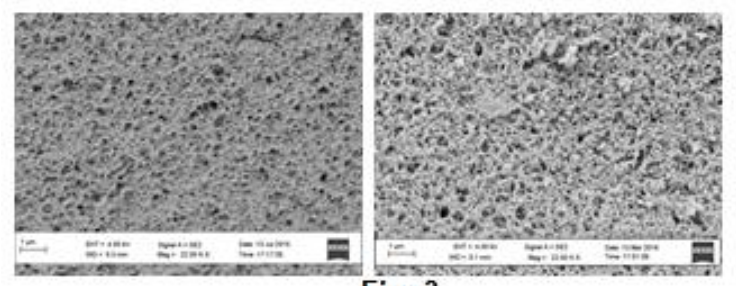

Fig. 3
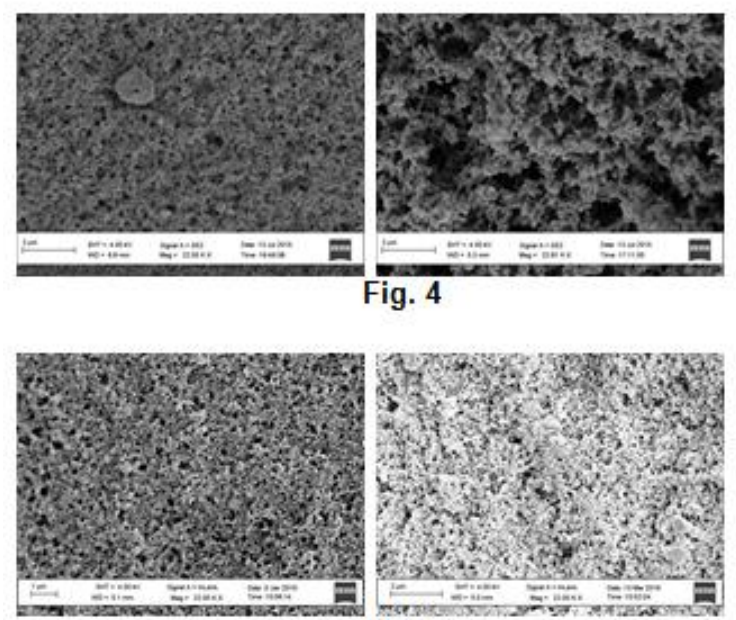

Fig. 5

\section{Conclusion:}

In conclusion, the significance of assessing the conformational stability was directly reflected on the ability of MIP3 to exhibit high reproducible specific binding compared to MIP2. Thus despite usually the literature relied on CE to select potential monomeric candidates for MIP synthesis, this paper could propose $T_{B}$ as a new efficient decisive parameter in synthesizing MIPs.

\section{References:}

1. Annamma, K.; Mathew, B. Design of 2,4-dichlorophenoxyacetic acid Imprinted Polymer with High Specificity And Selectivity. Mater Sci Appl. 2011, 2, 131-140.

2. Parmpi, P.; Kofinas, P. Biomimetic Glucose Recognition using Molecularly Imprinted Polymer Hydrogels. Biomaterials. 2004, 25, 1969-1973.

3. Muhammada, T.; Cuia, L.; Jide, W.; Piletska, E.; Guerreirob, A.; Piletsky, S. Rational design and synthesis of Water-Compatible Molecularly Imprinted Polymers for Selective Solid Phase Extraction of Amiodarone. Anal. Chim. Acta. 2012, 709, 98-104.

4. Khan, M.; Wate, P.; Krupadam, R. Combinatorial Screening of Polymer Precursors for preparation of Benzo[ $\alpha]$ pyrene Imprinted Polymer: an ab initio Computational Approach. J Mol Model. 2012, 18, 1969-1981. 
5. Li, X.; Zhong, S.; Chen, L.; Whittaker, A. Computer Simulation and Preparation of Molecularly Imprinted Polymer Membranes with Chlorogenic Acid as Template. Polym Int. 2011, 60, 592-598.

6. Carraher, Charles E. Polymer Chemistry, $7^{\text {th }}$ ed.; Taylor \& Francis: Florida, 2007. 


\title{
Evaluation of particle sizing by analytical centrifugation using real- world materials for the implementation of the EC definition of the term "nanomaterial"
}

\author{
C. Ullmann, ${ }^{1 *}$ I. Rio, ${ }^{2}$ D. Mehn, ${ }^{2}$ D. Gilliland, ${ }^{2}$ W. Wohlleben, ${ }^{3}$ R. Koeber,${ }^{4}$ F. Babick ${ }^{1}$ \\ ${ }^{1}$ Dresden University of Technology, Dresden, Germany \\ ${ }^{2}$ Joint Research Centre, Directorate F-Health, Consumers and Reference Materials F2, Ispra, Italy \\ ${ }^{3}$ BASF SE, Ludwigshafen, Germany \\ ${ }^{4}$ Joint Research Centre, Directorate F-Health, Consumers and Reference Materials F6, Geel, Belgium
}

\begin{abstract}
This study evaluates the performance for the grouping of particulate substances with analytical centrifugation (AC). Performance tests were carried out for a tri-modal polystyrene mixture and non-spherical $\mathrm{BaSO}_{4}$ materials in the nano and sub-micrometre size range on cuvette-type centrifuges $(470 \mathrm{~nm}$ and $865 \mathrm{~nm}$ ), disctype centrifuge $(405 \mathrm{~nm})$ and cuvette-type analytical ultracentrifuge $(670 \mathrm{~nm}$, RI measurement). The intermediate precision was determined by comparison of independently prepared daily triplicates for five days and data analysis in terms of ANOVA. Experimental results show that the selected illumination wavelengths of $470 \mathrm{~nm}$ and $865 \mathrm{~nm}$ have a little impact on the number-weighted median diameter, but affect the intermediate precision of the these values. However, the intermediate precision of the method is mainly determined by sample preparation up to $12.6 \%$ with respect to the number-weighted median diameter $\left(\mathrm{x}_{50,0}\right)$. Disc-type AC results show a precision (av. 10.6\%) and advantages in detecting nanoparticles but troubles with broadly distributed particle size distributions. The AUC shows a good precision (4.6 ...7.2\%) but the lowest numberweighted median.
\end{abstract}

Keywords: analytical centrifugation, evaluation, intermediate precision, nanomaterial detection, particle size

\section{Introduction}

In 2011 the European Commission (EC) recommended a definition of the term 'nanomaterial' [1]. This definition states that a material is considered a nanomaterial if more than $50 \%$ of the particles in the numberbased particle size distribution (PSD) $\mathrm{Q}_{0}(\mathrm{x})$ have one or more external dimensions in the size range of 1$100 \mathrm{~nm}$. The EC's recommendation of a definition includes aggregated, agglomerated and individual particles and will probably be implemented in several regulatory contexts, e.g. REACH. An overview on standardisation in particle characterization for safety assessment is given in [2].

The definition implementation is facilitated by a few measurement techniques that directly measure the numberweighted median particle diameter $\left(\mathrm{x}_{50,0}\right)$ of all primary particles - such as electron microscopy. Linsinger et al. [3] reviewed widely available measurement techniques in the nano range. Recently, Babick et al. [4] published a systematic evaluation of commonly used particle sizing techniques based on experimental data. In this context, identification of nanomaterials by AC implies that the reliability of the method reaches a certain level of interest and avoids false compliant results.

Any method description has to include sample preparation, measurement procedures, data analysis and, if necessary, conversion of measured PSDs to number-based PSDs. Turbidity AC intrinsically yields extinctionbased PSDs $\left(\mathrm{Q}_{\mathrm{ext}}(\mathrm{x})\right)$, yet the conversion of these PSDs to number-based ones $\left(\mathrm{Q}_{0}(\mathrm{x})\right)$ will be prone to errors after applying the Mie theory due to several simplifying assumptions. Babick and Ullmann [5] reconsidered the conversion to number-weighted PSDs theoretically and with regard to cuvette-type AC measurements results. ISO already standardised ACs operating with cuvettes [6] and those operating with disc-shaped rotors (disctype AC) [7]. According to ISO13318-2 [7], the typical particle size range is from about $0.1 \mu \mathrm{m}$ to $5.0 \mu \mathrm{m}$ for AC with turbidity detectors. Therefore, the reliability of optical AC techniques needs to be evaluated 
systematically for particle diameters below $100 \mathrm{~nm}$. Recently published studies examined the method's intermediate precision of the median diameter $\mathrm{x}_{50,0}$ and the size quantiles $\mathrm{x}_{10,0}$ and $\mathrm{x}_{90,0}$ of cuvette-type AC for particles in the nano and sub-micrometre size range [8]. This publication compares the main results to those obtained by disc-type AC and AUC [9].

\section{Experimental}

\subsection{Materials}

JRC purchased three monodisperse suspensions NANOSPHERE ${ }^{\mathrm{TM}}$ 3050, 3100, 3350 from Thermo Scientific (Fremont, USA) and provided a tri-modal polystyrene suspension for qualification of AC instruments. The certified diameters $-46 \pm 2 \mathrm{~nm}, 100 \pm 3 \mathrm{~nm}$ and $350 \pm 6 \mathrm{~nm}(\mathrm{k}=2)$ - were determined by transmission electron microscopy. The ratio of fractions was $0.5: 1: 1$ by mass and the suspension was further diluted with deionised water to a solid content of $2.5 \mathrm{~g} / \mathrm{kg}(0.24 \mathrm{vol} \%)$. The density used is $1.05 \mathrm{~g} / \mathrm{cm} 3$ and the refractive index is 1.59 .

The Joint Research Centre (JRC) of the European Commission provided two grades of $\mathrm{BaSO}_{4}$ powders IRMM381 (non-nano grade) and IRMM-387 (nano grade).

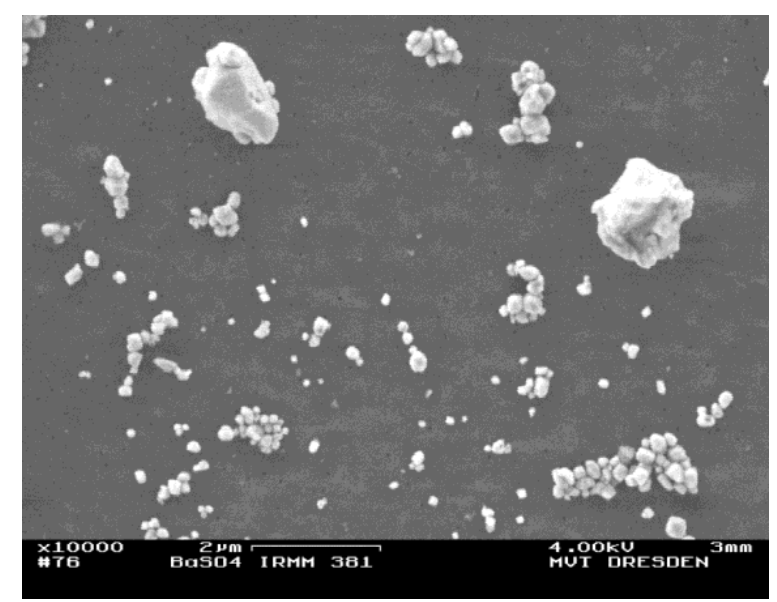

Figure 1: SEM image of IRMM-381

The IRMM-387 powder was sourced from an OECD sponsorship program repository and comes from the same batch as used in the EU-project NANOREG. Both materials passed tests on homogeneity and stability and the JRC classified them as representative test materials [10]. The manufacturer stated the particle density $4.4 \mathrm{~g} / \mathrm{cm} 3$ for both grades of $\mathrm{BaSO}_{4}$. Both, the substance IRMM-381 and the substance IRMM-387 consist mainly of single constituent particles, but also contains small amounts of agglomerates and aggregates. For the substance IRMM-381, SEM images show that the particle diameters are between $80 \mathrm{~nm}$ and $2 \mu \mathrm{m}$ (cf. Figure 1). The size range of IRMM-387 is from a few nanometres up to rare particles with diameters of about $200 \mathrm{~nm}$ (cf. Figure 2). 


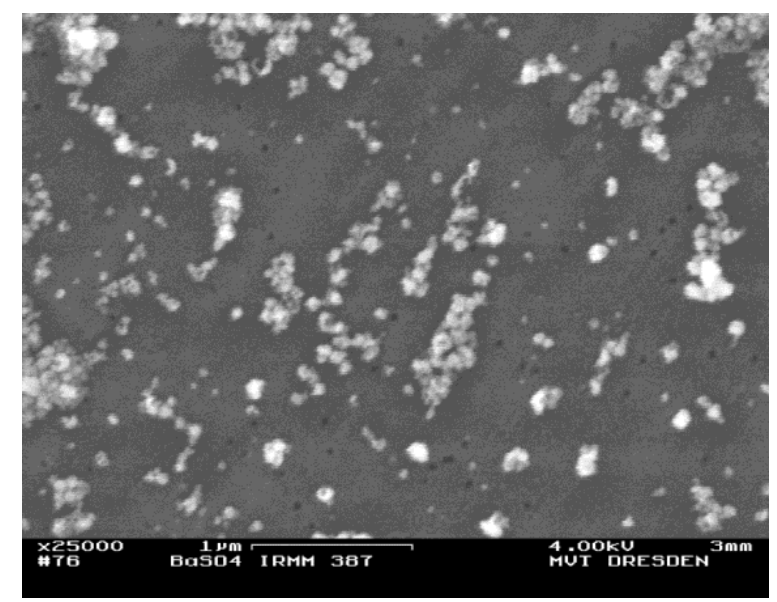

\subsection{Preparation of suspensions}

Figure 2: SEM images of IRMM-387

Suspensions of the two grades of $\mathrm{BaSO}_{4}$ were prepared by suspending powder in sodium hexametaphosphate solution. The suspensions were prepared in accordance to harmonized protocols with ultrasonic devices, volume specific energy input approx. $1600 \mathrm{~J} / \mathrm{ml}$ [11]. The pre-dispersed tri-modal polystyrene suspension $(0.24 \mathrm{vol} \%)$ was not diluted.

\subsection{Analytical centrifugation instruments}

Four instrument of analytical centrifugation technologies with optical detectors are tested: Cuvette-type centrifuges LUMiSizer 610 (illumination at 470nm), LUMiSizer 651 (865nm), disc-type centrifuge DC24000UHR (405nm) and cuvette-type analytical ultracentrifuge XL-I ProteomeLab Version (670nm). The AUC measures the relative refractive index increment and this principle leads intrinsically to a mass-based PSD $\left(\mathrm{Q}_{3}(\mathrm{x})\right)$. The other instruments monitor the turbidity and yield extinction-based PSDs $\left(\mathrm{Q}_{\mathrm{ext}}(\mathrm{x})\right)$. The conversion of extinction-based PSD to number-based ones $\left(\mathrm{Q}_{0}(\mathrm{x})\right)$ was done by applying the Mie theory.

\subsection{Determination of intermediate precision}

The method's intermediate precision was determined in accordance to ISO 5725:1994 [12] regarding the determination of the number-weighted median diameter $\mathrm{x}_{50,0}$. Within this study, the institutes compared results of independently prepared daily triplicates $\left(\mathrm{s}^{2} \mathrm{r}\right)$ of five days $\left(\mathrm{s}^{2}\right.$ day $)$ of both materials (cf.

Figure 3). The precision $(1 \sigma)$ of the number-based median diameter $\mathrm{x}_{50,0}$ was evaluated by principles of analysis of variance (ANOVA) in terms of relative standard deviation (RSD).

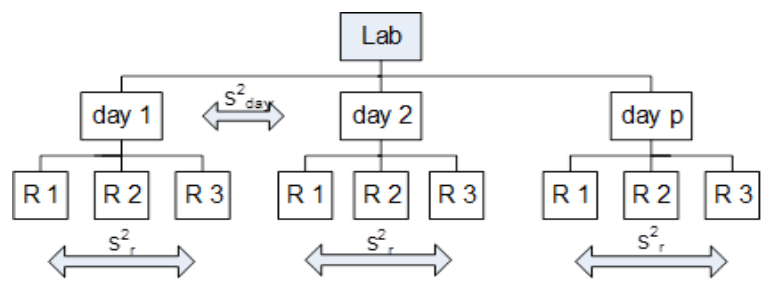

Figure 3: Design of experiments for determination of intermediate precision $(\mathrm{p}=5)$

\section{Results}

\subsection{Qualification}

Trueness tests with tri-modal polystyrene particle suspensions show consistent results for all AC techniques regarding the comparison of modal diameter $\mathrm{x}_{\text {mod,0 }}$ accuracy (cf. Figure 4 ). 


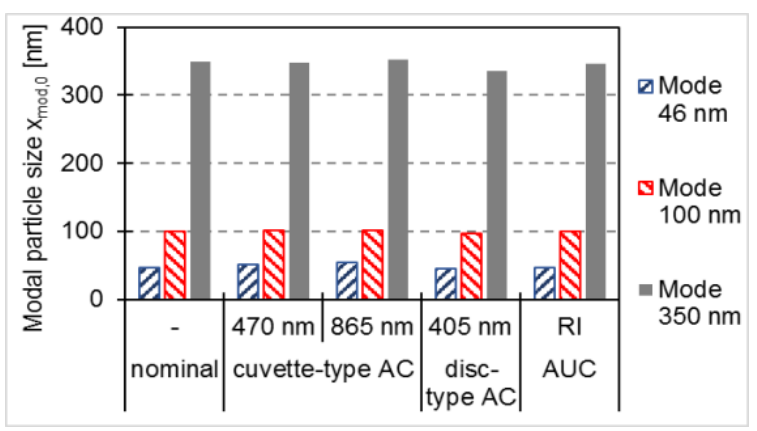

Figure 4: Comparison of mode accuracy $\mathrm{x}_{\mathrm{mod}, 0}$ of tri-modal polystyrene suspension $\mathrm{AC}$ measurements.

While particle size determination by TEM is associated with dried particles only and therefore determined without hydrodynamic effects, the number-based mode is an accepted value for the evaluation of the cuvettetype AC measurement technique. Therefore, some degree of discrepancy is expected and the deviation goes in the expected direction.

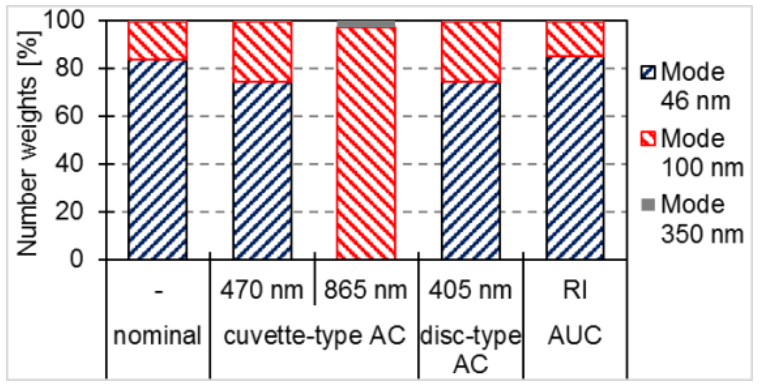

Figure 5: Comparison of mode's number quantity $\mathrm{Q}_{0}\left(\mathrm{x}_{\mathrm{mod}, 0}\right)$ of tri-modal polystyrene suspension $\mathrm{AC}$ measurements.

The comparison of particle number quantity shows a satisfying agreement between the AC techniques, except for cuvette-type AC operating with NIR illumination (cf. Figure 5). The measurements at $865 \mathrm{~nm}$ wavelength lead to a high underestimation of the $46 \mathrm{~nm}$ fraction due to the low turbidity of the suspension by illumination at NIR and could be determined in an advanced way of data analysis only.

\subsection{Intermediate precision of AC method}

Tests on real-world polydisperse $\mathrm{BaSO}_{4}$ particles lead to divergent median diameters $\mathrm{x}_{50,3}$ and $\mathrm{x}_{50,0}$ and their RSDs (cf. Figure 6-7).

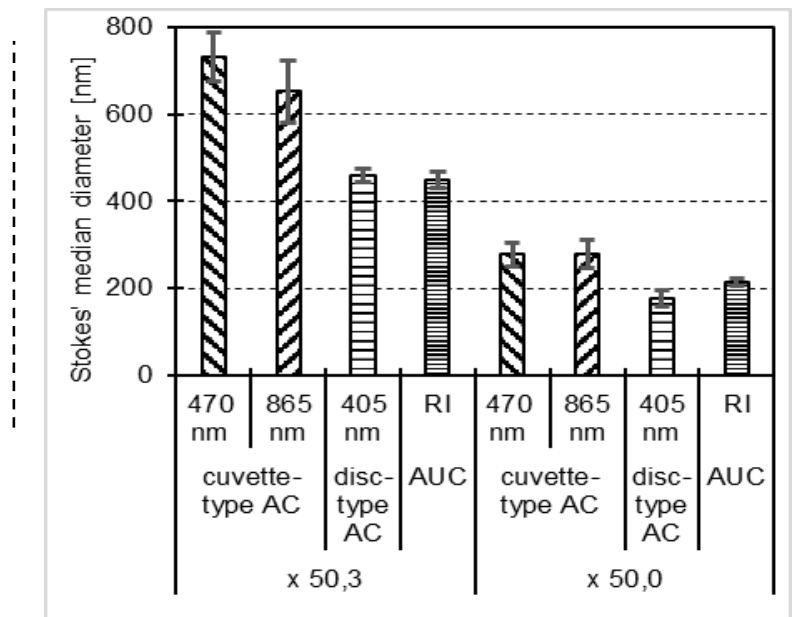

Figure 6: Comparison of volume-based median diameter $\mathrm{x}_{50,3}$ and number-based median diameter $\mathrm{x}_{50,0}$ of IRMM-381 
Studies on cuvette-type AC show that the intermediate precisions of number-based median diameter $\mathrm{x}_{50,0}$ and of the volume-based median diameter $x_{50,3}$ are slightly affected by illumination wavelength (RSD 9.4 ..12.6\%), but no impact on value of $\mathrm{x}_{50,0}$ is obvious for both grades of $\mathrm{BaSO}_{4}$ particles. The intermediate precision of the number-based median diameter $\mathrm{x}_{50,0}$ obtained by disc-type $\mathrm{AC}$ is similar to the intermediate precision obrtained by cuvette-type AC (RSD 10.3...10.9\%). There is a comparably good resolution of nanoparticle fractions, yet studies on IRMM-381 show that applicability range troubles for highly polydisperse PSDs. Studies on AUC with RI-detector show relatively low uncertainty of median diameter $\mathrm{x}_{50,0}$ (RSD: 4.6..7.2\%), but for nanograde $\mathrm{BaSO}_{4}$ also slightly lower number-based median diameter than for the other AC techniques $(22 \mathrm{~nm}$ instead of $44 \ldots 45 \mathrm{~nm}$ ).

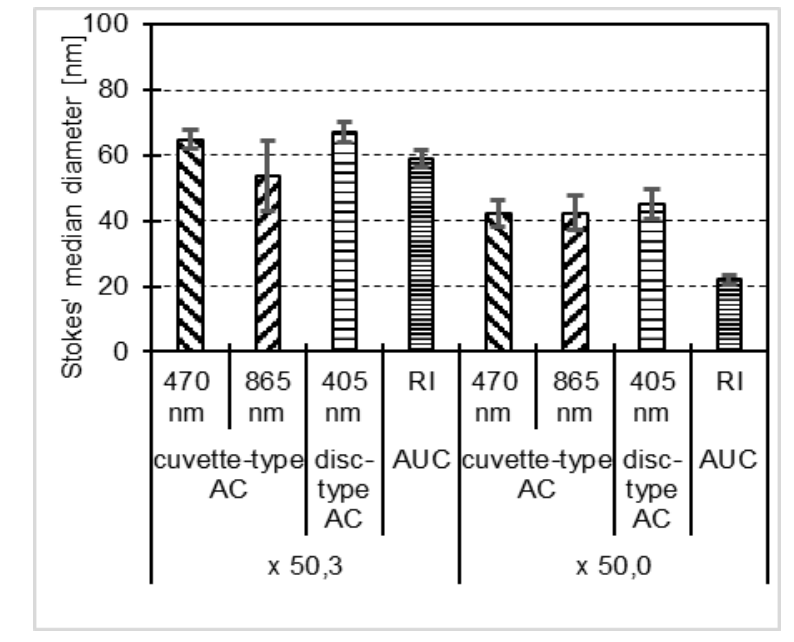

Figure 7: Comparison of volume-based median diameter $\mathrm{x}_{50,3}$ and number-based median diameter $\mathrm{x}_{50,0}$ of IRMM-387

Impact of sample preparation is the main source of uncertainty for all AC types. The erosion of ultra-sonic devices, the complexity of energy input calibration, suspension stability and sedimentation issues and subsampling errors mainly cause this uncertainty. The state of agglomeration is at least as important and requires validity checks.

\section{Conclusions}

This metrological study shows high consistency of all AC techniques with regard to number-based median diameter $\mathrm{x}_{50,0}$ of non-ideal real-world materials in the nano and non-nano size range. Intermediate precision of AC method (incl. sample preparation) of $\mathrm{x}_{50,0}$ is below $13 \%$ RSD for all configurations examined, except using NIR illumination. Analytical centrifugation facilitates material classification according to the EC's recommendation of a definition of the term nanomaterial on a screening level according to the decision tree of NanoDefine. Otherwise, borderline cases require further analysis. In every case, the material needs to be reasonably well dispersed. A challenge is to avoid contamination of samples during sample preparation particularly by erosion of dispersing tools.

\section{Acknowledgements:}

This document presents results of the European project NanoDefine, which has received funding from the EC's Seventh Framework Programme under Grant Agreement $n^{\circ}$ 604347. In addition, the authors would like to thank LUM GmbH for providing the instrument LUMiSizer® 651. Moreover, the authors thank Petra Fiala (TUD) for taking the SEM images.

\section{References:}

1. European Commission (2011/696/EU) Official J. Eur. Union. (2011) 54:38-40

2. M. Stintz, D. Goehler. Procedia Engineering 102 (2015) 233 - 239 
3. T.P.J. Linsinger, G. Roebben, D. Gilliland, L. Calzolai, F. Rossi, N. Gibson, C. Klein, Report EUR 25404 (2012)

4. F. Babick, J. Mielke, W. Wohlleben, S. Weigel, V.-D. Hodoroaba. J. Nanopart. Res. 18:158 (2016)

5. F. Babick, C. Ullmann. Powder Technology 301 (2016) 503-510

6. ISO 13318-1:2001, Geneva, 2001

7. ISO 13318-2:2007, Geneva, 2007

8. C. Ullmann, F. Babick, R. Koeber, M. Stintz. Powder Technology 319 (2017) 261-270

9. D. Mehn, I. Rio, D. Gilliland, P. Schuck, W. Wohlleben: Analytical ultra centrifugation with fixed and ramped speed for nanomaterial identification. In prep. (2017)

10. G. Roebben, K. Rasmussen, V. Kestens, T. P. J. Linsinger, H. Rauscher, H. Emons, H. Stamm, J. Nanopart. Res. 2013, 15:1455

11. D. Gilliland, F. Pianella, I. Rio, K. Loeschner, M. Correia, E. Verleysen, J. Mast, C. Ullmann. Technical Report D2.3 NanoDefine, Wageningen 2016, available at www.nanodefine.eu

12. ISO 5725-3:1994, Including technical corrigendum 1:2001. Geneva, 1994 


\title{
Inexpensive and Rapid Vesicles Synthesis for Biomedical Applications
}

\author{
Chandra Has ${ }^{1}$, Sopan M. Phapal ${ }^{2}$, P. Sunthar ${ }^{3}$ \\ ${ }^{1,2,3}$ Department of Chemical Engineering, Indian Institute of Technology Bombay (IITB) \\ Powai, Mumbai 400076, India \\ ${ }^{1}$ chandrahas@iitb.ac.in, ${ }^{2}$ sopan56@iitb.ac.in, ${ }^{3}$ P.Sunthar@iitb.ac.in
}

\begin{abstract}
The major focus of the present study is to produce size-controlled liposomes in a short period of about $15 \mathrm{~min}$. The method is based on the mixing of two miscible phases, i.e., the lipid phase in which lipid/ethanol is premixed with water, and the aqueous phase. The mixing of two phases resulting the self-assembly of lipid molecules to generate liposomes. This novel method produces monodisperse DOPC liposomes of size about 170-220 nm with polydispersity less than 0.2 as obtained by dynamic light scattering instrument (DLS). Since the method is the single step, rapid, inexpensive and also free from any harsh chemical and mechanical treatments, it can be easily employed for the delivery of drugs and genetic materials.
\end{abstract}

Keywords: Self-assembly; diffusive mixing; phospholipid membranes; monodisperse liposomes; biomedical applications

\section{Introduction}

Liposomes (or lipid vesicles) have been emerged as one of the most advantageous delivery vehicles for the various drugs. They are the spherical structures composed of either a single lipid bilayer or multiple layers separated by aqueous phase. Liposomes can entrap hydrophilic agents into their inner aqueous core and lipophilic ones within the lipid bilayer region(s) [1]. In general, the entrapment efficacy of liposomes for lipophilic agents is always higher than hydrophilic. The reason behind the vesicles formation is the accumulation of lipid molecules that yield entropically favorable conditions of minimal free energy in an energetically favorable manner [2]. Liposome size and size distribution are two important factors for in vivo applications. It has been shown that liposomes of size in the range of 100 to $150 \mathrm{~nm}$ have higher potential in order to target them to the tumor sites through the enhanced permeation and retention (EPR) mechanism [3].

To date, there have been several conventional methods developed for the liposomes synthesis but most of them involve complex and tedious technique. Here, we present an inexpensive, simple, and rapid approach for preparing monodisperse liposomes. Liposomes can be observed within just $15 \mathrm{~min}$ and have a long shelf life. The principle of vesicles formation is based on the diffusion driven process of two miscible phases $[4,5]$. The process involves the self-assembly of lipids into liposomes as ethanol speedily diffuses into the aqueous phase. In this method, the size of the liposome can be easily controlled by regulating the temperature parameter.

\section{Materials and Methods}

\subsection{Materials}

The lipid 1, 2-Dioleoyl-sn-glycero-3-phosphocholine (DOPC) in powder form was obtained as a generous gift from Lipoid GmbH (Ludwigshafen, Germany). The solvent ethanol was obtained from Merck. One end closed glass capillaries (i.d.: $1 \mathrm{~mm}$, o.d.: $1.4 \mathrm{~mm}$, length: $100 \mathrm{~mm}$ ) and sealant (paraffin film/wax) were procured from a local trader. Long SS needles $(25 \mathrm{G} \times 10 \mathrm{~cm})$ were purchased from Victor- G. \& company (India).

\subsection{Vesicle preparation}

The main components of the experimental are: a small diameter capillary, long needles with glass syringes, and sealants such as paraffin film or wax (Error! Reference source not found.). Using syringe-needle assembly, water is first filled up to about $4 \mathrm{~cm}$ from the closed end. Lipid phase containing a mixture of lipid-ethanol and water (volumetric ratio of ethanol and water is one) is taken in another 


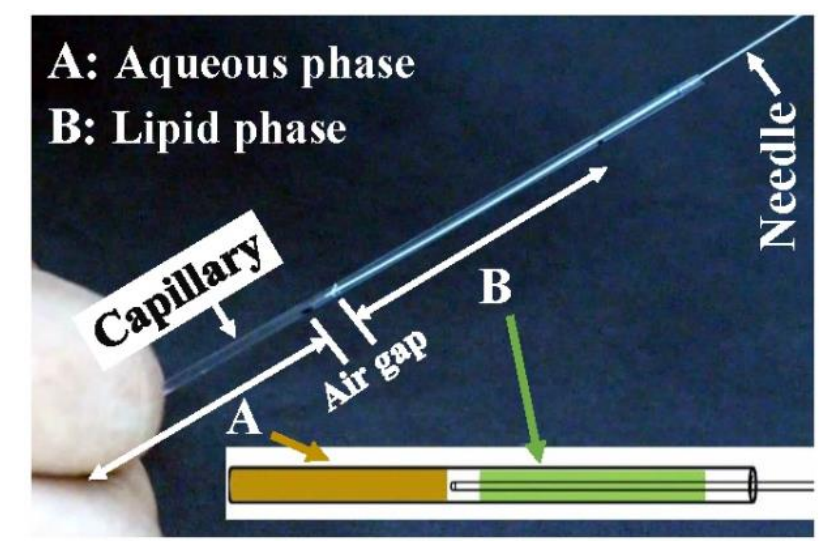

Fig. 1: Capillary setup. The two phases (aqueous and lipid phases) are filled one by one in one end closed glass capillary with keeping a small air gap between them. The air gap is removed using a thin, long needle.

Thereafter, the open end is sealed with a sealant.

syringe and filled up to $4 \mathrm{~cm}$ by leaving a small air gap (about $5 \mathrm{~mm}$ ) between the two phases. In this way, the overall ethanol proportion in the whole solution is maintained at $25 \%$ (by volume). However, it can be taken around $30 \%$ (by volume) but above this, the vesicle could no longer be retained as small globular shapes appear [6]. The two miscible phases are brought into contact by removing the air gap through another syringe-needle assembly. After generating the interface, the open end of the capillary is sealed with paraffin film or wax. The capillary set up is then kept in an incubator where the temperature is set above the lipid phase transition temperature.

The capillary is oriented vertically as well as horizontally in an incubator. However, only horizontal orientation gives monodisperse vesicles. In this method, liposomes appear in the form of milky white colour (turbid colour) which can be observed in a few moments. Within just $15 \mathrm{~min}$ more than $80-90 \%$ length of the capillary becomes turbid and rest of the part takes another 30-45 min. However, this small unturbid part can be carefully removed and the turbid part can be taken for the liposome characterization. Liposomes produced by this method are characterized by dynamic light scattering (Malvern instruments Zen 1600, Malvern, UK) and scanning electron microscopy (SEM) in Cryo-mode.

\section{Results and discussions}

\subsection{Vesicle size and size distribution}

From the dynamic light scattering (DLS), the $z$-average hydrodynamic diameter of DOPC liposome at $24{ }^{\circ} \mathrm{C}$ is found to be $170 \pm 4.3 \mathrm{~nm}$ with a PDI of $0.14 \pm 0.03$. A single peak in the intensity distribution of vesicle size (average diameter) shows the presence of monodisperse particles in the sample (Fig. 2a). The Cryo-SEM result in Fig. $2 b$ shows that the liposomes are spherical in structures. In Fig. 2c, the number particle size distributions obtained from DLS and Cryo-SEM are compared, and they are found to be in close agreement. Here, lipid concentration in ethanol is $10 \mathrm{mg} / \mathrm{ml}$.

\subsection{Effect of temperature and lipid concentration on vesicle size}

At above the phase transition temperature $\left(T_{\mathrm{m}}\right)$ of the lipid, vesicle can be successfully prepared. For DOPC, $T_{\mathrm{m}}=$ $-17^{\circ} \mathrm{C}$. In this study, setup is kept in a temperature-controlled incubator for 45 min at different temperatures. We observe the significant influence of temperature on the vesicles size, as shown in Fig. $2 \mathrm{~d}$.

We also assess the effect of lipid concentration on vesicle size. For this, various lipid concentrations are prepared in ethanol, i.e., 10, 20, and $50 \mathrm{mg} / \mathrm{ml}$. Unlike conventional ethanol injection method where vesicle size increases with increasing lipid concentration [7], we observe no significant influence of lipid concentration on the liposome size and size distribution (Table 1). Here, higher concentrations only lead more number of vesicles hence higher volume fraction which can be evidenced by a comparatively more turbid suspension inside the capillary 


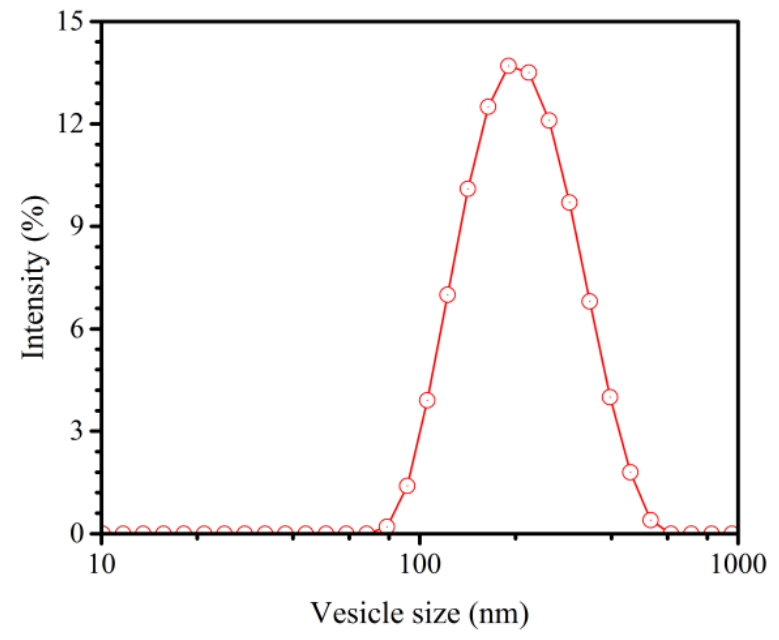

(a)

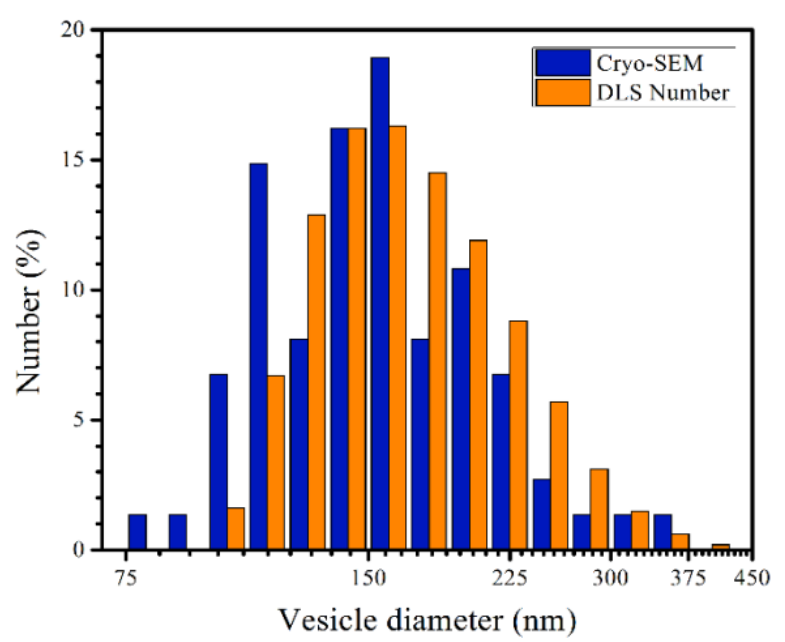

(c)

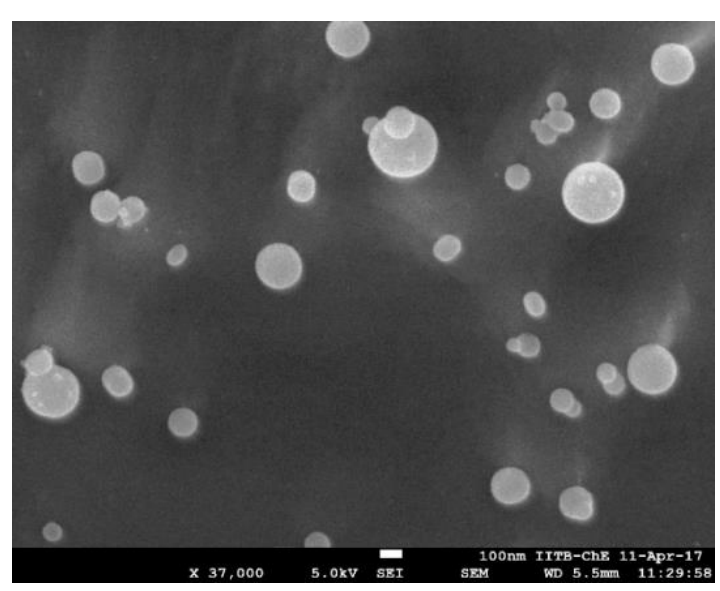

(b)

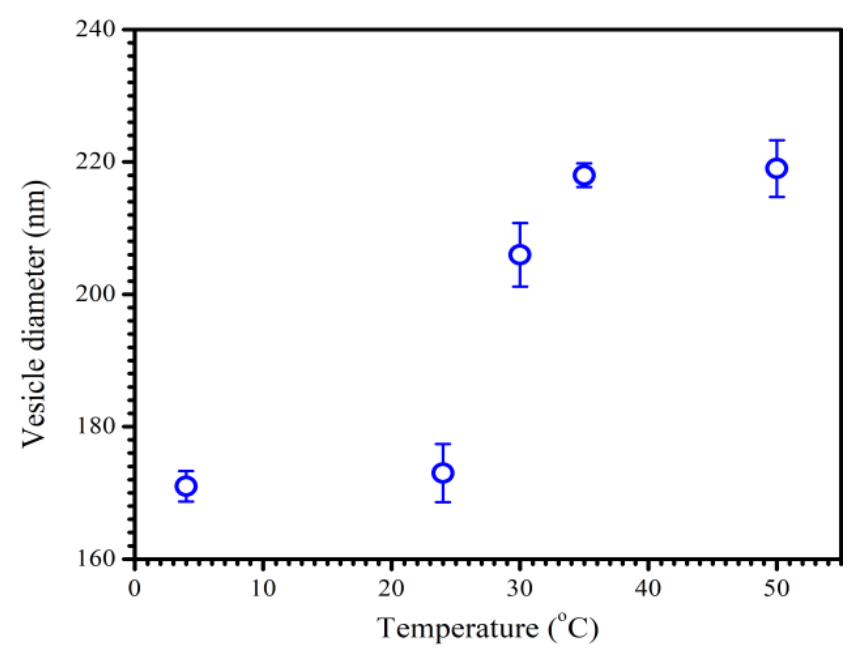

(d)

Fig. 2: Vesicle characterization and temperature effect. (a) The monodisperse size distribution of DOPC liposome in term of intensity distribution obtained in dynamic light scattering (DLS). (b) Shows the morphology of DOPC liposomes from Cryo-SEM result. The scale bar is $100 \mathrm{~nm}$. (c) Number distribution of liposome size from Cryo-SEM is compared with the number distribution that obtained from DLS measurement for the same sample. For Cryo-SEM, number distribution is obtained from 64 liposomes in similar images and including the present one. (d) Shows the vesicle size variation with temperature. As shown, size is almost invariant up to about $25^{\circ} \mathrm{C}$ and above which diameter start increasing gradually. However, size again almost becomes constant after about $30^{\circ} \mathrm{C}$.

Table 1: Effect of lipid concentration (C) on vesicle size (D) and polydispersity index (PDI) with standard deviation (SD) at $24{ }^{\circ} \mathrm{C}$.

\begin{tabular}{|c|c|c|}
\hline $\mathrm{C}(\mathrm{mg} / \mathrm{ml})$ & $\mathrm{D}(\mathrm{nm}) \pm \mathrm{SD}$ & PDI $\pm \mathrm{SD}$ \\
\hline 10 & $171 \pm 4.4$ & $0.14 \pm 0.03$ \\
\hline 20 & $169 \pm 5.1$ & $0.16 \pm 0.02$ \\
\hline 50 & $179 \pm 5.6$ & $0.20 \pm 0.02$ \\
\hline
\end{tabular}

\subsection{Mechanism of vesicle formation}

In the present method, two phases are quickly interdiffused (ethanol and water are highly miscible) and fully solvated lipids self-assemble, leading to the generation of monodisperse liposomes [8]. Here, lipid molecules into 
the vicinity of the diffusive ethanol-water interface initially aggregate into a disk-like membranes, which grow by the fusion process with other disks. Subsequently, disks curve and close upon themselves to form liposomes [5, 9].

\section{Conclusion}

We have successfully presented a rapid, inexpensive and a single step liposome preparation technique that is suitable for producing monodisperse vesicles with low PDI $(<0.2)$, requiring no post processing steps such as sonication or extrusion. The vesicle of size ranging $170-220 \mathrm{~nm}$ can be easily achieved by only regulating the temperature parameter whereas size remains invariant when lipid concentration is varied. The liposome can be observed within just $15 \mathrm{~min}$. We anticipate that with this simple and efficient technique, the finding of novel liposomal formulations can be better investigated and more widely administered in clinical settings.

\section{Acknowledgements}

The authors gratefully acknowledge SAIF and CRNTS for providing the lab facilities.

\section{References}

[1] V. P. Torchilin, "Recent advances with liposomes as pharmaceutical carriers," Nat. Rev. Drug Discov., vol. 4, pp. 145-160, 2005.

[2] D. A. Balazs and W. T. Godbey, "Liposomes for use in gene delivery," J. Drug Deliv., vol. $2011,2010$.

[3] C. Dhand, M. P. Prabhakaran, R. W. Beuerman, R. Lakshminarayanan, N. Dwivedi, and S. Ramakrishna, "Role of size of drug delivery carriers for pulmonary and intravenous administration with emphasis on cancer therapeutics and lung-targeted drug delivery," RSC Adv., vol. 4, pp. 32673-32689, 2014.

[4] S. M. Phapal and P. Sunthar, "Influence of micro-mixing on the size of liposomes self-assembled from miscible liquid phases," Chem. Phys. Lipids, vol. 172, pp. 20-30, 2013.

[5] S. M. Phapal, C. Has and P. Sunthar, "Spontaneous formation of Single Component Liposomes from a Solution," Chem. Phys. Lipids, vol. 205, pp. 25-33, 2017.

[6] J. Mou, J. Yang, C. Huang and Z. Shao, "Alcohol induces interdigitated domains in unilamellar phosphatidylcholine bilayers," Biochemistry, vol. 33, pp. 9981-9985, 1994.

[7] J. M. H. Kremer, M. W. Van der Esker, C. Pathmamanoharan and P. H. Wiersema, "Vesicles of variable diameter prepared by a modified injection method," Biochemistry, vol. 16, pp. 3932-3935, 1977.

[8] D. D. Lasic, "The mechanism of vesicle formation," Biochem J., vol. 256, pp. 1-11, 1988.

[9] A. Jahn, W. N. Vreeland, D. L. DeVoe, L. E. Locascio and M. Gaitan, "Microfluidic directed formation of liposomes of controlled size," Langmuir, vol. 23, pp. 6289-6293, 2007. 


\title{
Thermoelectric Properties of Zinc Antimonide Thin Film
}

\author{
C. Woo, ${ }^{1, *}$ H. Shim, ${ }^{1}$ S. $\operatorname{Han}^{1}$ \\ ${ }^{1}$ Korea Institute Of Machinery \& Materials, Department of Nanomechanics, Daejeon, Korea
}

\begin{abstract}
:
The zinc antimonide compound $\mathrm{Zn}_{\mathrm{x}} \mathrm{Sb}_{\mathrm{y}}$ is one of the most efficient thermoelectric materials known at high temperatures, due to its exceptional low thermal conductivity. For this reason, it continues to be the focus of active research, especially on its glass-like atomic structure. However, before practical use in actual surroundings such as near a vehicle manifold, it is imperative to analyze the thermal reliability of these materials. Herein we present the thermal cycling behavior of $\mathrm{Zn}_{\mathrm{x}} \mathrm{Sb}$ thin films in nitrogen $\left(\mathrm{N}_{2}\right)$ purged or ambient atmosphere. $\mathrm{Zn}_{\mathrm{x}} \mathrm{Sb}_{\mathrm{y}}$ thin films were prepared by co-sputtering, and reached a power factor of $1.39 \mathrm{~mW} \mathrm{~m}^{-1} \mathrm{~K}^{-2}$ at $321{ }^{\circ} \mathrm{C}$. The high temperature in-situ XRD patterns and TEM results shows that the degradation in thermoelectric performance of $\mathrm{Zn}_{\mathrm{x}} \mathrm{Sb}_{\mathrm{y}}$ thin film with increasing number of thermal cycling comes from structural, morphological and compositional changes. In particular, the fluidity of $\mathrm{Zn}$ atoms in $\mathrm{Zn}_{\mathrm{x}} \mathrm{Sb}_{\mathrm{y}}$ thin film matrix during the thermal cycling give rise to morphological and compositional changes, such as nano inclusions or voids, which leads to degradation of the thermoelectric performance over thermal cycling. Moreover, these changes are more developed under atmospheric measurement condition, which generate thermally decomposed $\mathrm{Zn}$ or other impurity phase. For instance, the $\mathrm{ZnO}$ coating layer, which comes from oxidation of thermally decomposed $\mathrm{Zn}$ from the $\mathrm{Zn}_{\mathrm{x}} \mathrm{Sb}_{\mathrm{y}}$ thin film, keep the thermoelectric performance for a while by blocking the $\mathrm{Zn}$ evaporation from the $\mathrm{Zn}_{\mathrm{x}} \mathrm{Sb}_{\mathrm{y}}$ thin film. However, $\mathrm{Zn}_{\mathrm{x}} \mathrm{Sb}_{\mathrm{y}}$ thin film eventually was broken by growth of $\mathrm{Zn}$ fiber to relive the thermal stress, which followed by Zn agglomeration with further atmospheric thermal cycling. These results provide insight for the needs for the proper encapsulation of the $\mathrm{Zn}_{\mathrm{x}} \mathrm{Sb}_{\mathrm{y}}$ surface that does not degrade thermoelectric performance with repeated thermal cycling.
\end{abstract}

Keywords: zinc antimonide, thermoelectric thin film, thermal cycle, thermoelectric properties and measurement, RF magnetron co-sputtering.

\section{Result and Discussion}

Figure 1a shows the XRD pattern that was extracted from the in-situ high temperature XRD data between RT and $270{ }^{\circ} \mathrm{C}$. After heat treatment, even their different sputtering condition of substrate temperature at RT vs. $100{ }^{\circ} \mathrm{C}$, they shows very similar XRD patterns unlike XRD patterns of as deposited film. However, according to a quantitative analysis based on XRD data, the proportion of $\mathrm{Zn}$ in the type $B$ thin film is $\sim 2.6$ times higher than that of type A. As mentioned before, this excessive $\mathrm{Zn}$ component hampered the phase transition toward $\beta-\mathrm{Zn}_{4} \mathrm{Sb}_{3}$ and it may mirror the increased resistivity ( $\rho$ ) of the thin film over $200{ }^{\circ} \mathrm{C}$ (Figure 1c). Actually, in the temperature range between RT to $133{ }^{\circ} \mathrm{C}$ the measured $\rho$ of type $B$ is much lower than that of RT, because of excessive Zn chunks on the thin film as we mentioned in Figure $1 \mathrm{~b}$. However, above $178{ }^{\circ} \mathrm{C}$ these $\mathrm{Zn}$ residuals are a bad influence on the conductivity of the thin film. As shown in Figure $2 \mathrm{~b}$, the as grown $\mathrm{Zn}$ chunks break the thin film and this might lead to a decrease in the conductivity of the thin film with increasing temperature. Consequently, type $B$ shows a lower power factor (P.F.) value of $0.92 \mathrm{~mW} \mathrm{~m}{ }^{-1} \mathrm{~K}^{-2}$ as compared to type $A$, which has a P.F. of $1.39 \mathrm{~mW} \mathrm{~m}^{-1} \mathrm{~K}^{-2}$ due to its lower conductivity at $321{ }^{\circ} \mathrm{C}$, even though it has a slightly higher Seebeck coefficient $(\alpha)$ value than type A. Moreover, as shown in the in-situ high temperature XRD pattern, type $B$ contains less $\beta-\mathrm{Zn}_{4} \mathrm{Sb}_{3}$ component than type $A$, and the thermoelectric efficiency is also poor due to its higher thermal conductivity ( $\kappa$ ), which is related to the excessive $\mathrm{Zn}$ component. Actually, since we estimated $z T$ values versus temperature for the type $A$ thin film based on values of the corresponding bulk materials, of course the samples indicate a maximum $z T$ value of 1.26 at $321{ }^{\circ} \mathrm{C}$, which is associated with the maximum content of $\beta-\mathrm{Zn}_{4} \mathrm{Sb}_{3}$ in the thin film, and a minimum $z T$ value of $2.64 \times 10^{-4}$ at $24{ }^{\circ} \mathrm{C}$ when the maximum $\mathrm{Zn}$ content was in it. However, the P.F. value of type $B$ above $427{ }^{\circ} \mathrm{C}$ is higher than that at RT. This is related to the interesting sudden $\rho$ drop above $426{ }^{\circ} \mathrm{C}$, which is possibly due to melted $\mathrm{Zn}$ (the melting point of $\mathrm{Zn}$ is $\sim 420{ }^{\circ} \mathrm{C}$ ) connected to percolating paths along the grain boundaries. The porous morphologies along the grain boundaries are shown in the TEM image, which was obtained after measurement of TE properties, and also support the likelihood of this phenomenon. 

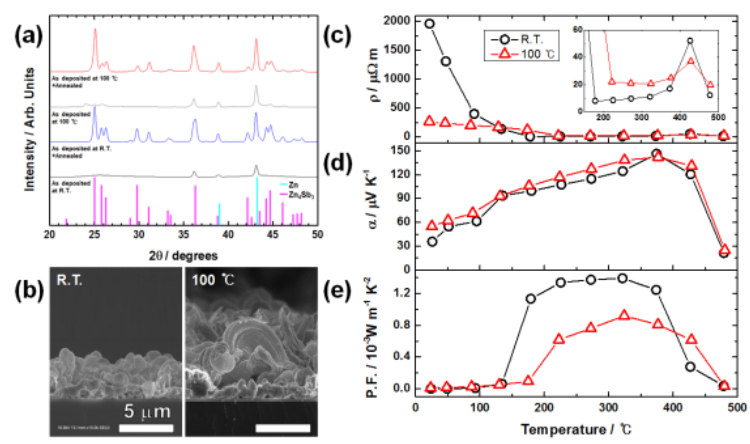

Figure 1: XRD patterns of sample deposited on the substrate at (a) room temperature (type A, gray line, $\mathrm{Zn}+$ amorphous $\mathrm{Zn} 4 \mathrm{Sb} 3\left(\mathrm{a}-\mathrm{Zn}_{4} \mathrm{Sb}_{3}\right)$ ), and $100{ }^{\circ} \mathrm{C}$ (type $B$, light gray line, $\left.\mathrm{Zn}+\mathrm{Zn}_{4} \mathrm{Sb}_{3}\right)$. The blue and red lines indicate the XRD patterns of type $A$ and $B$ samples, respectively, after annealing. (b) Cross sectional FESEM images of type $A$ (left) and $B$ (right) samples. Thermoelectric properties of $\mathrm{Zn}_{\mathrm{x}} \mathrm{Sb}_{\mathrm{y}}$ thin film samples as a function of temperature: (c) resistivity ( $\rho$ ), (d) Seebeck coefficient $(\alpha)$, and (e) Power factor (P.F.) data.

\section{References:}

1. Bell, E. (2008) Cooling, Heating, Generating Power, and Recovering Waste Heat with Thermoelectric Systems. Science, 321, 1457-1461.

2. Venkatasubramanian, R.; Siivola, E.; Colpitts, T.; O’Quinn, B. (2001) Thin-Film Thermoelectric Devices with High Room-temperature Figures of Merit. Nature, 413, 597-602.

3. Snyder, J.; Christensen, M.; Nishibori, E.; Caillat, T.; Iversen, B. (2004) Disordered Zinc in $\mathrm{Zn}_{4} \mathrm{Sb}_{3}$ with Phonon-Glass and Electron-Crystal Thermoelectric Properties. Nat. Mater.3, 458- 463.

4. Sun, Y.; Christensen, M.; Johnsen, S.; Nong, V.; Ma, Y.; Sillassen, M.; Zhang, E.; Palmqvist, C.; Bottiger, J.; Iversen, B. (2012) Low-Cost High-Performance Zinc Antimonide Thin Films for Thermoelectric Applications. Adv. Mater. 24, $1693-1696$.

5. Caillat, T.; Fleurial, P.; Borshchevsky, A. (1997) Preparation and Thermoelectric Properties of Semiconducting $\mathrm{Zn}_{4} \mathrm{Sb}_{3}$. J. Phys. Chem. Solids 58, 1119- 1125.

6. Toberer, S.; Rauwel, P.; Gariel, S.; Tafto, J.; Snyder, J. (2010) Composition and the Thermoelectric Performance of $\beta-\mathrm{Zn}_{4} \mathrm{Sb}_{3}$. J. Mater. Chem. 20, 9877- 9885. 


\title{
Effects of electrical breakdown on the photocurrent generation in thin- film single-walled carbon nanotubes
}

\author{
W. Dumnernpanich ${ }^{1}$, M. Sapankaew ${ }^{1}$, J. Jantawong ${ }^{2}$, N. Penpondee ${ }^{2}$, C. Hruanun ${ }^{2}$, Y. Jompol ${ }^{1}$ \\ ${ }^{1}$ Department of Physics, Faculty of Science, Mahidol University, Bangkok, Thailand \\ ${ }^{2}$ Thai Microelectronic Center, Chachoengsao, Thailand
}

\begin{abstract}
We have observed the reduction in the photocurrent after an electrical breakdown of a thin-film semiconducting single-walled carbon nanotube (s-SWCNT) field-effect transistor (FET). The photocurrent $\left(I_{\mathrm{ph}}\right)$ is defined as the excess current between light and dark conditions and studied under ambient temperature with different light intensities. Before the electrical breakdown, the measured current-voltage $(I-V)$ characteristic exhibits non-linear behaviour upon changing wavelength and light intensity, suggesting that electrons tunnel across Schottky barriers of metal-SWCNTs junctions. The photocurrent before the electrical breakdown $I_{\mathrm{ph}}^{\text {Before }}$ was found nearly 15 times higher than that of the post-breakdown. After the electrical breakdown, the $I$ - $V$ characteristic becomes linear, but we have seen almost no change in the measured current when increasing light intensities to the maximum power. This observation indicates that charge-carrier transport in the SWCNT film is governed by electron diffusion through the Schottky barriers. Our results also suggest that the photocurrent generation in SWCNTs depends strongly on built-in electric field at the Schottky barriers and inter-tube junctions. Further verification of chargecarrier separation in such devices can be demonstrated by electrical noise measurements.
\end{abstract}

Single-walled carbon nanotubes (SWCNTs) have potential applications for light harvesting in photovoltaic research including solar cells due to their excellent electronic and optical properties, whose bandgap energies are tunable within the solar spectrums [1-5]. Several reports have shown that their electrical conductivity of individual SWCNTs and SWCNT film also responses to infrared (IR) absorption spectra upon changing light intensities [610]. However, there still be argument about photocurrent generation in SWCNTs whether this is resulted by exciton or thermal effects. In hybrid SWCNTs-polymer matrix (polycarbonate - PC), Pradhan et al. have observed a dramatic improvement in the electrical conductivity (up to $4.56 \%$ in contrast to the pure HiPco produced SWCNT film) under the IR illumination [11]. This improvement in the photoresponse is attributed to a combination of thermal and photoexcitation effects upon percent weight (\%wt) of the SWCNT mass fraction. In their work, the change in the photoconductivity in pure SWCNT film is dominated by thermal effect, while in SWCNT-polymer nanocomposite is due to the photo (exciton) effect. According to Pradhan et al., the PC in such mixed SWCNT-polymer structure could give rise to exciton dissociations via (i) the local temperature of the SWCNT-embedded insulating PC matrix that increase thermal energy to dissociate excitons, and (ii) the local electric field at the SWCNT-PC interface, which is essential for exciton dissociation. These explanations agree well with our experimental results on the photocurrent generation mechanism in thin-film SWCNTs.

In this work, we report on the observation of an electrical effect to the photocurrent in SWCNT film upon illumination of near infrared (NIR) light. By dropping a large DC voltage across SWCNT film, inter-tube junctions and metal-nanotube junctions are thermally diminished and so the junction breakdown is achieved via Joule heating effect [12-14]. We found that after the junction breakdown, the current-voltage $(I-V)$ characteristic becomes linear, indicating that charge-carrier diffusions dominate over the tunnelling and hopping transports across the SWCNT film [15]. Moreover, with illumination of light over the entire SWCNT film we have seen almost no change in the photocurrent. This finding suggests that the potential barrier to the electron tunnelling between nanotubes and metal-nanotube junctions plays an important role in the photocurrent generation mechanism.

The devices in this study have been implemented into field-effect transistor (FET) structure. The SWCNTs are assembled in between 

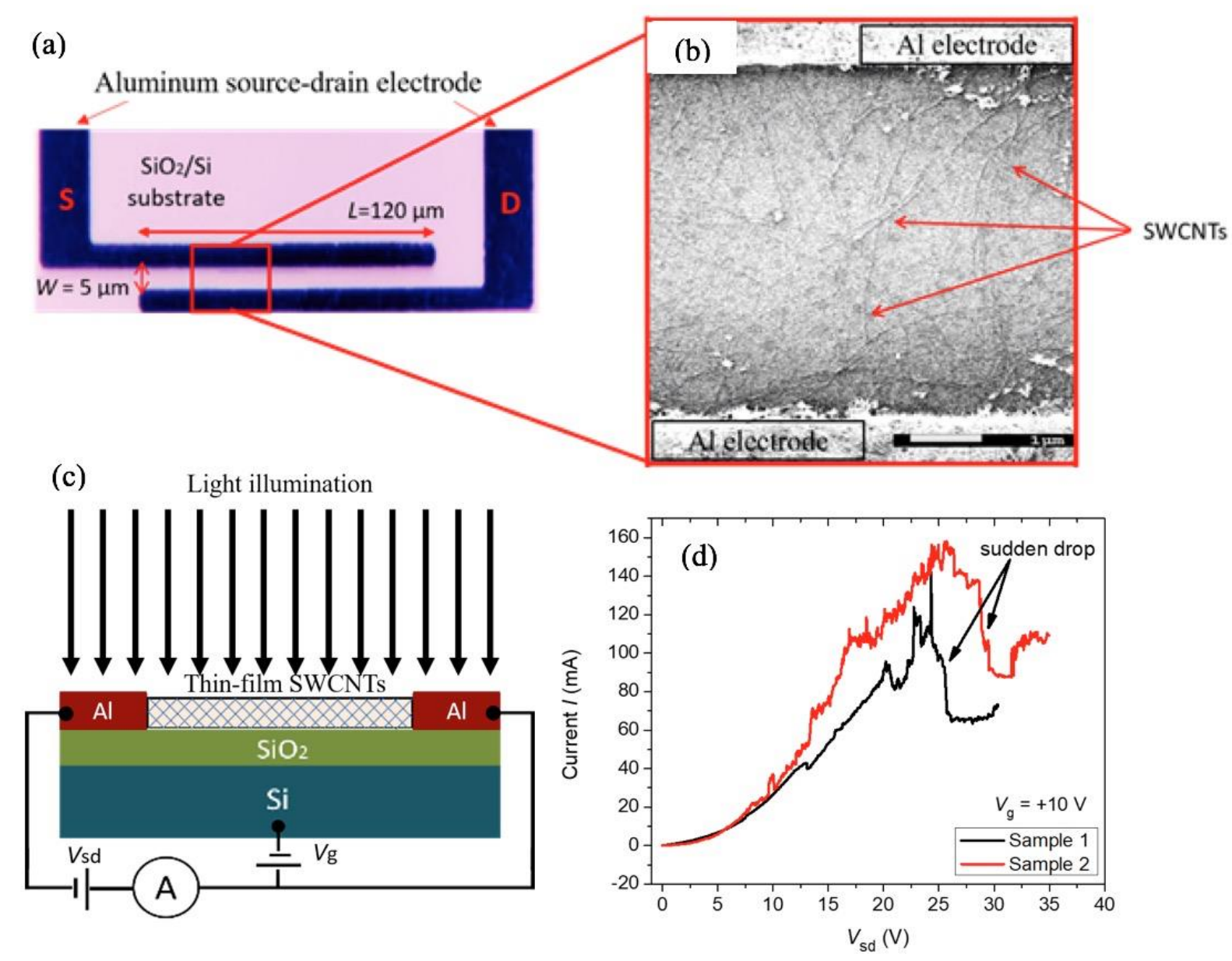

Figure 1: (a) An optical image of a conducting channel used in this work. Al electrode used for source and drain contacts. (b) SEM image of the SWCNTs film in an area of the conducting channel shown in (a). (c) Circuit diagram of the photocurrent measurement showing source-drain voltage $\left(V_{\mathrm{sd}}\right)$, gate bias $\left(V_{\mathrm{g}}\right)$, and light projection. Quartz tungsten halogen $(\mathrm{QTH})$ lamp together with a bandpass filter used to select a particular wavelength for the photoexcitation. (d) Typical $I$ - $V$ characteristics indicating a sudden drop in the current at a large source-drain bias at $V_{\mathrm{g}}=+10 \mathrm{~V}$ of Sample 1 and 2 (other samples not shown here).

source-drain electrode by dielectrophoresis process to form a conducting channel on $\mathrm{SiO}_{2} / \mathrm{Si}$ substrate, as shown in Fig. 1a. We used 99\% purity of semiconducting SWCNTs commercially available from NanoIntegris Inc., with a dilution $0.3 \mu \mathrm{g} / \mathrm{mL}$ in toluene solution. The solution contains non-uniform nanotubes with average diameter of $1.4 \mathrm{~nm}$ and length of $1-5 \mu \mathrm{m}$. Source-drain electrodes were made of titanium nitride (TiN) layer (40 nm thick) and aluminum (Al) layer (60 nm thick). After the DEP process, a network of SWCNTs was formed in the gap between two parallel Al electrodes, as shown in Fig. 1b. The channel gap has length $L=120 \mu \mathrm{m}$ long with width $W=5 \mu \mathrm{m}$ wide.

Two-terminal current-voltage characteristics were carried out using Keithley 2401 source-meter unit together with Keithley 2200 DC power supply for gate bias. The measurements were performed at ambient temperature under EPS150COAX DC parametric test system from Cascade Microtech Inc., with a computer-controlled LabVIEW programs. To perform photocurrent measurements, we used research light source from Newport Company with quartz tungsten halogen (QTH) lamp to produce broad spectrum from visible to near infrared light output at 200 $\mathrm{nm}-2500 \mathrm{~nm}$. The output of the beam is $33-\mathrm{mm}$ diameter which is collimated onto the entire sample that glued on a printed circuit broad (PCB), shown in Fig. 1c. Intensity of the beam is controlled by OPS-Q250 power supply. To select a particular excitation of photon energy, we inserted a bandpass filter with a narrow centered frequency between the beam output and the sample. The incident power of the illumination after the filter was measured using Newport power meter model 843-R with a silicon detector. 
The electrical breakdown was obtained by applying a large voltage across source-drain electrode until a sharp drop in current was seen. As shown in Fig. 1d, at high-bias voltage $V>+20 \mathrm{~V}$ the current increases up to $0.14-0.16$ $\mathrm{mA}$ and a sudden drop occurs, which indicates a large removal of junction resistance. These values can turn to a power of $3 \mathrm{~mW}-4 \mathrm{~mW}$ required in these samples. The breakdown current and voltage depend on the number and formation of the nanotubes in the channel and the choice of electrode material. We performed this electrical breakdown at non-zero gate bias e.g., $V_{\mathrm{g}}=+10 \mathrm{~V}$ for all devices at which the semiconducting channel is in very low conducting mode or in the "Off" state in the FET configuration. In this work, we use electrical breakdown in order to improve the device performance when operated as FET by electrically removing a series resistance from thin-oxide layer, acting as Schottky barrier. Such an effect could yield a higher On/Off current ratio in the FET operation. The breakdown current has also induced high heat in the center of the nanotubes that likely breaks in the middles [14]. When breaking down, we applied a gate bias to protect the semiconducting nanotubes in the channel.

Figure $2 \mathrm{a}$ and $2 \mathrm{~b}$ show comparisons in the $I-V$ characteristics and trans-conductance measurement, respectively, before and after an electrical breakdown. The $I-V$ characteristic of Sample 1 clearly shows a transformation from nonlinearity to almost linearity over $V_{\mathrm{sd}}= \pm 2 \mathrm{~V}$ after breakdown. There is also an increase in the current for any given biases. This implies that the channel becomes more conductive. Here, the resistance of Sample 1 after breakdown reduces from $\sim 1.6 \mathrm{M} \Omega$ (evaluated at $V_{\text {sd }}=+2 \mathrm{~V}$ ) to $\sim 1 \mathrm{M} \Omega$. The reduction in the junction resistance reveals the decrease in the tunnel barriers throughout the film and metal-nanotube. As a result of the electrical breakdown, transconductance $\left(g_{\mathrm{m}}=\mathrm{d} I / \mathrm{d} V_{\mathrm{g}}\right)$ of the samples has relatively increased at a fixed bias voltage at $+2 \mathrm{~V}$. The current $I$ was measured as a function of gate bias $V_{\mathrm{g}}$, applied at $\mathrm{Si}$ doped substrate from $-10 \mathrm{~V}$ to $+10 \mathrm{~V}$. The channel is $p$-type semiconducting FET. The transconductance is determined from the slope of $I-V_{\mathrm{g}}$ for $V_{\mathrm{g}}$ from +5 $\mathrm{V}$ to $+10 \mathrm{~V}$. After the breakdown, $g_{\mathrm{m}}$ is increased from $122 \mathrm{nS}$ to
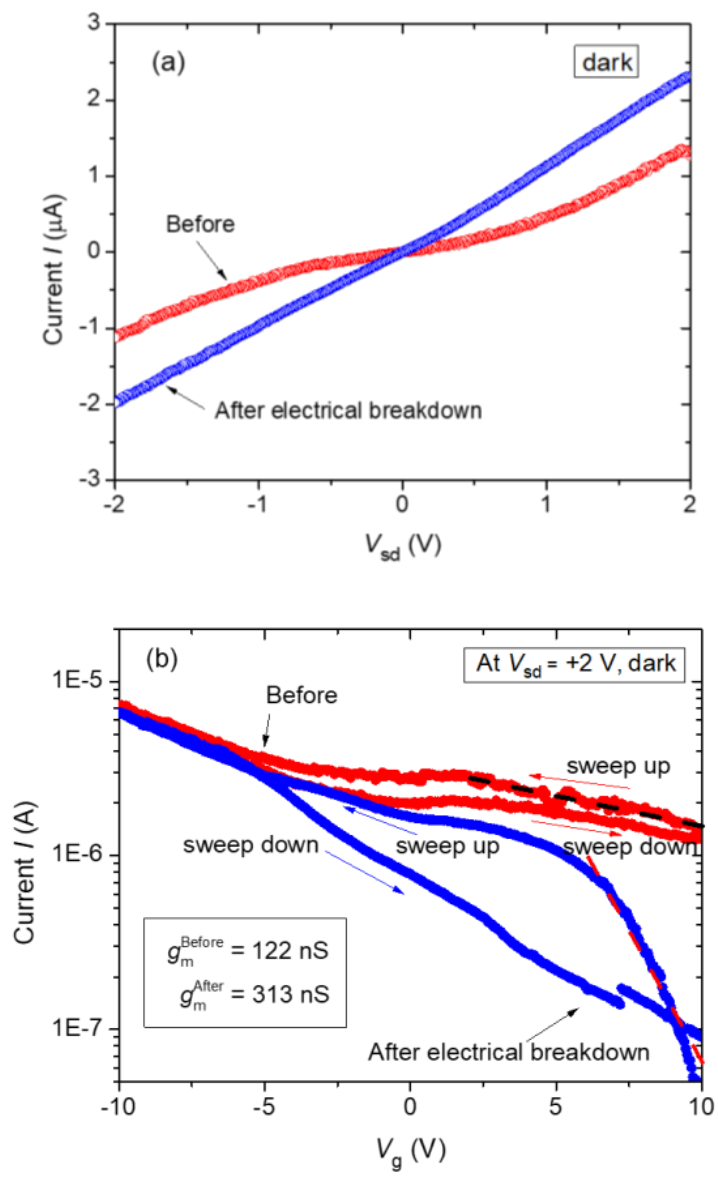

Figure 2: (a) The $I-V$ characteristics before and after electrical breakdown. (b) Transconductance $\left(g_{\mathrm{m}}\right)$ measurement before and after electrical breakdown showing current as function of $V_{\mathrm{g}}$ for both sweep up and down in the dark conduction. 
$313 \mathrm{nS}$ of sweep up. The current ratio evaluated at $V_{\mathrm{g}}= \pm 10 \mathrm{~V}$ applied at Si doped substrate also increases by $\sim 1$ order of magnitude, as shown in Fig. 2b. This enhancement suggests that there is a reduction of series resistance at the metal-nanotube interfaces in our FET channel.

Optical excitation into the SWCNT film has been carried out using QTH light source to investigate photocurrent generation mechanism at room temperature. We exploited a bandpass filter at center wavelength $800 \mathrm{~nm}$ irradiating onto the entire chip. In Fig. 3a, current versus time for a given source-drain bias at $V_{\mathrm{sd}}=+2 \mathrm{~V}$ is shown. The measured current stability was obtained over 5 minutes at ambient temperature and with different light conditions. Before the electrical breakdown, the current was centered around $1.25 \mu \mathrm{A}$ without illumination (dark) and increase to $\sim 3.8 \mu \mathrm{A}$ when illuminated with power $4.5 \mathrm{~mW}$ (light). The increase in the measured current corresponds to the resistance drop by about $750 \mathrm{k} \Omega$ and that yields the excess current (the photocurrent, $I_{\mathrm{ph}}$ ) before breakdown to be $I_{\mathrm{ph}}^{\text {Before }}=+2.5 \mu \mathrm{A}$ in Sample 1. Other samples (Sample 2 and 3) have $I_{\mathrm{ph}}^{\text {Before }}$ around $0.1 \mu \mathrm{A}-1$ $\mu \mathrm{A}$, as shown in Fig. 3b. In post-electrical breakdown, we repeated this photoexcitation experiment with the same light conditions and found that those samples are almost insensitive to the light. As seen in Sample 1, we induced the photocurrent after the electrical breakdown to be $I_{\mathrm{ph}}^{\mathrm{After}}=+0.17 \mu \mathrm{A}$, while in Sample 2 and 3 these values are very small compared to that in the pre-electrical breakdown. The main reason behind this difference is that the latter two samples has multiple breakdown, which may reduce the number of the nanotubes in the channel. Such a small change in the measured current after breakdown yields a resistance change by about $18 \mathrm{M} \Omega$ in Sample 2, which indicates that this device is insensitive to the light since one needs to have a large decrease in the sample resistance in order to increase the photocurrent by $1 \mu \mathrm{A}$. This means that one has to have high intensity radiation to excite charge carriers in the nanotubes. In Fig. 3b, the effect of electrical breakdown has influenced on the photocurrent as seen in the Sample 2 and 3. Unlike in Sample 1, the measured current of Sample 2 and 3 after the breakdown is lower than the dark current of their pre-breakdown condition. This means that the channels are more resistive after a number of electrical breakdown have been repeated. The increase in the resistance in Sample 2 and 3 suggests that not only does the breakdown process reduce the metal-oxide layer at metal-nanotube contacts but it also burns out the nanotubes in the conducting channel.

Figure 4a shows the measured current before the breakdown process as a function of light intensities with different wavelengths. We see that the measured current is increased and saturated at $P \approx 2.5 \mathrm{~mW}$. After the breakdown, we have used the maximum power for the light condition. Figure $4 \mathrm{~b}$ shows a comparison of the measured current between pre- and post-electrical breakdown of Sample 1 as a function of time given to the source-drain voltage under light illumination. In the pre-electrical breakdown, one could see charging effect when the applied voltage swings between $+2 \mathrm{~V}(\mathrm{On})$ and $0 \mathrm{~V}(\mathrm{Off})$. The estimated charging time $(\tau)$ was deduced using exponential fit by equation, $I=I_{0}+A \exp (-\mathrm{t} / \tau)$. In the pre-electrical breakdown, $\tau$ was found $4 \mathrm{~s}$ while in the post-electrical breakdown much shorter $\tau$ was obtained to $7 \mathrm{~ms}$. Such fast voltage response implies that the sample after the breakdown becomes more conductive.

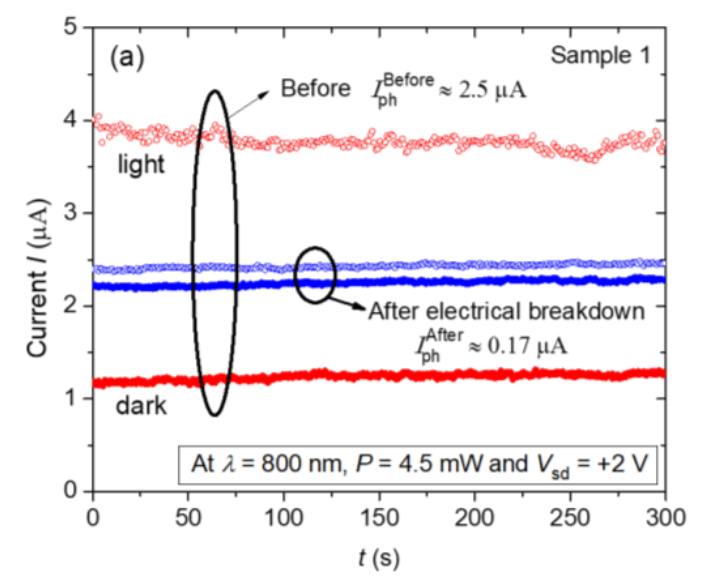




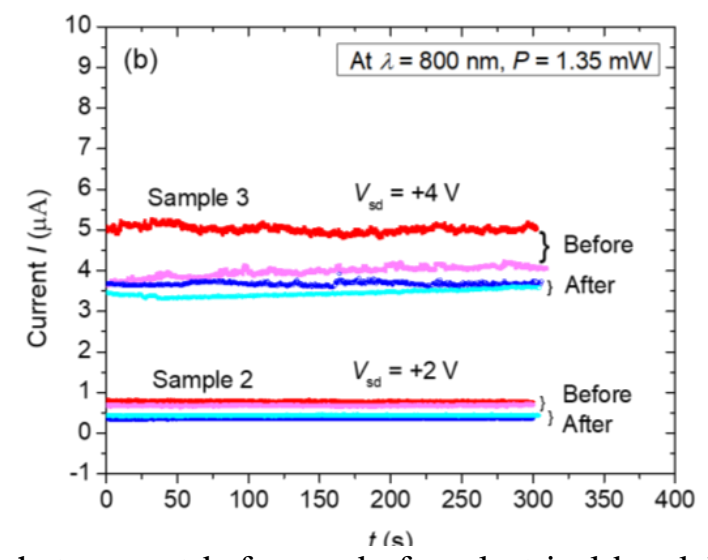

Figure 3: (a) Time dependent of photocurrent before and after electrical breakdown of Sample 1 by illuminated light at wavelength $\lambda=800 \mathrm{~nm}, P=4.50 \mathrm{~mW}$ and $V_{\mathrm{sd}}=+2 \mathrm{~V}$. (b) Similar results showing for Sample 2 and 3 at $P$ $=1.35 \mathrm{~mW}$.
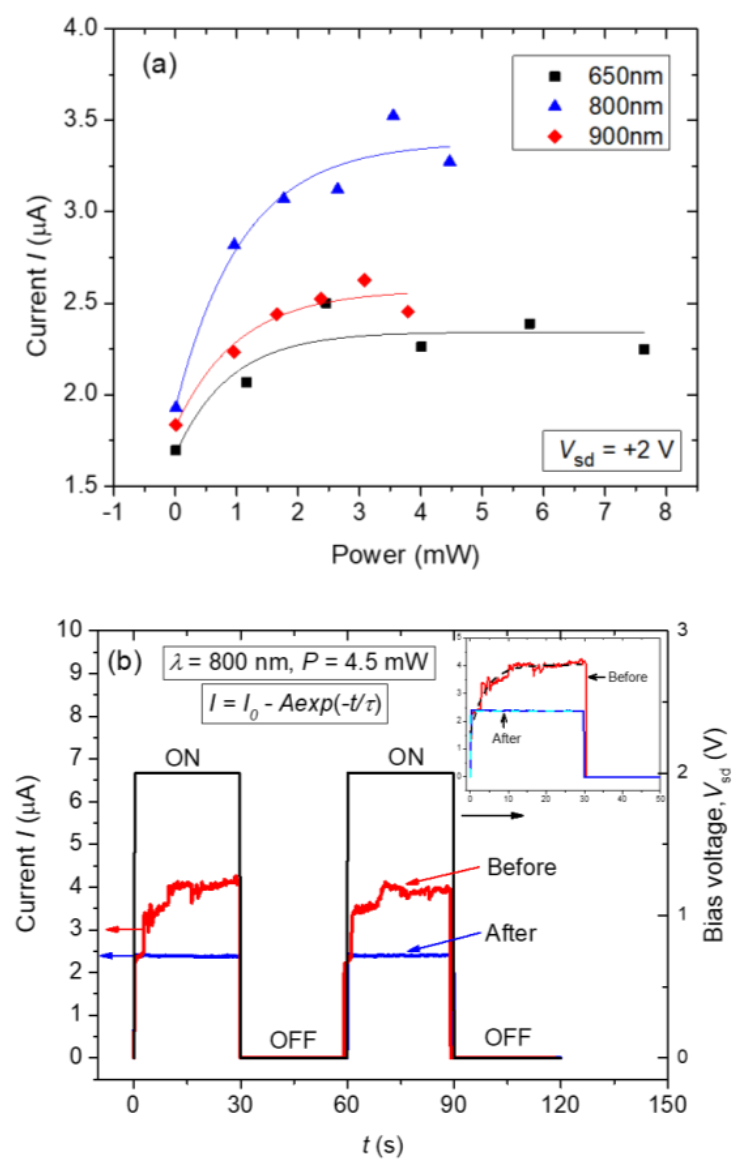

Figure 4: (a) The measured current as a function of light intensities with different wavelengths at $V_{\text {sd }}=+2 \mathrm{~V}$. (b) Time dependent of the measured current of Sample 1 under light illumination (left axis) at $\lambda=800 \mathrm{~nm}$ and $P=4.5$ $\mathrm{mW}$. Right axis shown Source-drain voltage as a function of time between $+2 \mathrm{~V}$ and $0 \mathrm{~V}$. Inset: exponential fit of the measured current $I$ as shown in the main graph.

In bulk SWCNT-polymer matrix [11], the photovoltaic effect in this structure was explained by two photocurrent generation mechanisms with IR radiation: (i) Photo effect or exciton effect for which electron-hole pairs are dissociated by a large electric field from local barriers or built-in electric field [10,16,17], and (ii) Thermal effect for which electrons and holes recombine and radiate heat which results in the resistance change [10]. The latter is known as the bolometric effect. The exciton dissociations are essential to create free electrons for photocurrent in semiconducting SWCNTs [18,19]. Like in hybrid carbon nanotube-polymer systems [20-22], the electric field at 
the CNT-conjugated polymer interface is mainly responsible for exciton dissociation generated in conjugated polymers (e.g., polythiophene) by photoexcitation. Also, in the SWCNT-PC nanocomposite, the SWCNTs are coated with an electrically insulating PC thin layer which acts as a potential barrier for charge hopping [23-27]. The enhanced local-electric field at the SWCNT-PC interface could also help exciton dissociation [18].

These studies agree well with our experimental data that the junctions in our SWCNT film give rise to the built-in potential which requires for exciton dissociations. When the number of junctions reduces as a result of the electrical breakdown, poor photocurrent is seen. So the current generation in the SWCNT film should exploit local electric field from both inter-nanotube and metal-nanotube junctions upon illumination of light.

In conclusion, we have demonstrated that photocurrent generation in SWCNT film requires local-electric field from nanotube-nanotube and metal-nanotube junctions for the exciton dissociations. Our experiments reveal that the effects of electrical breakdown result in reducing metal-oxide contact resistance, heating and incorporating the nanotube junctions. As the number of junctions reduces by the electrical breakdown, the photocurrent current with light illumination is poor and shows no sign for light sensing, but the device characteristics are significantly improved. Metal-nanotube junctions are important and should dominate the exciton dissociation at Schottky barrier. To gain more qualitative indication, further noise measurements should be used to characterise chargecarrier transports between pre- and post-breakdown of the SWCNT film via the noise Fano factor.

\section{Acknowledgement}

This work is supported by the Junior Science Talent Project under National Science and Technology Development Agency (NSTDA). YJ acknowledges the funding from the Faculty of Science, Mahidol University.

\section{References}

1. Li, Y., Kodama, S., Kaneko, T. \& Hatakeyama, R. Harvesting infrared solar energy by semiconducting single-walled carbon nanotubes. Appl. Phys. Express 4, 065101(2011).

2. Tune, D. D. \& Shapter, J. G. The potential sunlight harvesting efficiency of carbon nanotube solar cells. Energy Environ. Sci. 6, 2572 (2013).

3. Blackburn, J. L. Semiconducting Single-Walled Carbon Nanotubes in Solar Energy Harvesting. ACS Energy Lett. 2, 1598 (2017).

4. Kozawa, D., Hiraoka, K., Miyauchi, Y., Mouri, S. \& Matsuda, K. Analysis of the photovoltaic properties of single-walled carbon nanotube/silicon heterojunction solar cells. Appl. Phys. Express 5, 042304 (2012).

5. Avouris, P., Chen, Z. \& Perebeinos, V. Carbon-based electronics. Nat. Nanotechnol. 2, 605 (2007).

6. Freitag, M., Martin, Y., Misewich, J., Martel, R. \& Avouris, P. Photoconductivity of single carbon nanotubes. Nano Lett. 3, 1067 (2003).

7. Qiu, X., Freitag, M., Perebeinos, V. \& Avouris, P. Photoconductivity Spectra of Single-Carbon Nanotube: Implications of the Nature of Their Excited States. Nano Lett. 5, 749 (2005).

8. Levitsky, I. A. \& Euler, W. B. Photoconductivity of single-wall carbon nanotubes under continuous-wave near-infrared illumination. Appl. Phys. Lett. 83, 1857 (2003).

9. Fujiwara, A. et al. Photoconductivity of single-wall carbon nanotube films. Carbon N. Y. 42, 919 (2004).

10. Itkis, M. E., Borondics, F., Yu, A. \& Haddon, R. C. Bolometric Infrared Photoresponse of Suspended SingleWalled Carbon Nanotube Films. Science. 312, 413 (2006).

11. Pradhan, B., Setyowati, K., Liu, H., Waldeck, D. \& Chen, J. Carbon nanotube - Polymer nanocomposite infrared sensor. Nano Lett. 8, 1142 (2008).

12. Pop, E. Energy dissipation and transport in nanoscale devices. Nano Res. 3, 147 (2010).

13. Wei, Y., Liu, P., Jiang, K., Liu, L. \& Fan, S. Breaking single-walled carbon nanotube bundles by Joule heating. Appl. Phys. Lett. 93, 023118 (2008).

14. Berger, C., Yi, Y., Gezo, J., Poncharal, P. \& Heer, W. A De. Contacts, non-linear transport effects and failure in multi-walled carbon nanotubes. 5, 158.1 (2003).

15. Zhao, J., Sun, H., Dai, S., Wang, Y. \& Zhu, J. Electrical breakdown of nanowires. Nano Lett. 11, 4647 (2011).

16. Wang, F. The Optical Resonances in Carbon Nanotubes Arise from Excitons. Science. 308, 838 (2005). 
17. Matsuoka, Y. et al. Temperature dependence of photoconductivity at $0.7 \mathrm{eV}$ in single-wall carbon nanotube films. Sci. Technol. Adv. Mater. 4, 47 (2003).

18. Spataru, C. D., Ismail-Beigi, S., Benedict, L. X. \& Louie, S. G. Excitonic Effects and Optical Spectra of Single-Walled Carbon Nanotubes. Phys. Rev. Lett. 92, 77402 (2004).

19. Perebeinos, V., Tersoff, J. \& Avouris, P. Scaling of excitons in carbon nanotubes. Phys. Rev. Lett. 92, 257402-1 (2004).

20. Kymakis, E. \& Amaratunga, G. A. J. Single-wall carbon nanotube/conjugated polymer photovoltaic devices. Appl. Phys. Lett. 80, 112 (2002).

21. Xu, Z., Wu, Y., Hu, B., Ivanov, I. N. \& Geohegan, D. B. Carbon nanotube effects on electroluminescence and photovoltaic response in conjugated polymers. Appl. Phys. Lett. 87, 263118 (2005).

22. Pradhan, B., Batabyal, S. K. \& Pal, A. J. Functionalized carbon nanotubes in donor/acceptor-type photovoltaic devices. Appl. Phys. Lett. 88, 093106 (2006).

23. Ding, W. et al. Direct Observation of Polymer Sheathing in Carbon Nanotube-Polycarbonate Composites. Nano Lett. 3, 1593 (2003).

24. Sankapal, B. R., Setyowati, K., Chen, J. \& Liu, H. Electrical properties of air-stable, iodine-doped carbonnanotube-polymer composites. Appl. Phys. Lett. 91, 10 (2007).

25. Kilbride, B. E. et al. Experimental observation of scaling laws for alternating current and direct current conductivity in polymer-carbon nanotube composite thin films. J. Appl. Phys. 92, 4024 (2002).

26. Barrau, S., Demont, P., Peigney, A., Laurent, C. \& Lacabanne, C. DC and AC Conductivity of Carbon Nanotubes - Polyepoxy Composites. Macromolecules. 36, 5187 (2003).

27. Sichel, E. K., Gittleman, J. I. \& Sheng, P. Transport properties of the composite material carbon-poly(vinyl chloride). Phys. Rev. B 18, 5712 (1978). 


\title{
The effect of the surfactant in the stability of NiO-nanofluids
}

\author{
Antonio Sánchez-Coronilla, ${ }^{1, *}$ Elisa I. Martín, ${ }^{2}$ Javier Navas, ${ }^{3}$ Teresa Aguilar, ${ }^{3}$ Roberto Gómez- \\ Villarejo, ${ }^{3}$ Juan Jesús Gallardo, ${ }^{3}$ Rodrigo Alcántara, ${ }^{3}$ Desireé De los \\ Santos, ${ }^{3}$ Iván Carrillo-Berdugo, ${ }^{3}$ Concha Fernández-Lorenzo ${ }^{3}$ \\ ${ }^{1}$ Seville University, Department of Physical Chemistry, Seville, Spain \\ ${ }^{2}$ Seville University, Department of Chemical Engineering, Seville, Spain \\ ${ }^{3}$ Cádiz University, Department of Physical Chemistry, Cádiz, Spain
}

Concentrating Solar Power (CSP) is one of the most interesting option as renewable energy today. One option in order to improve the efficiency of these plants is to enhance the thermal properties of the heat thermal fluid (HTF) by using nanofluids. In this sense, this study presents an analysis of the stability of $\mathrm{NiO}$ nanoparticles on a base fluid composed of the eutectic mixture of diphenyl oxide and biphenyl with Benzalkonium Chloride (BAC) and 1-Octadecanethiol (ODT) as surfactants. The base fluid is a eutectic mixture of biphenyl $\left(\mathrm{C}_{12} \mathrm{H}_{10}, 26.5 \%\right)$ and diphenyl oxide $\left(\mathrm{C}_{12} \mathrm{H}_{10} \mathrm{O}, 73.5 \%\right)$. Three nanofluid systems (75\% BAC, $75 \%$ ODT and 75\% BAC-75\% ODT) were prepared and analysed. The stability of the nanofluid was analysed using different techniques UV-vis spectroscopy, particle size and $\zeta$ potential measurements. It is worth noting that the stability of the nanofluids improved with the use of BAC. UVvis spectra showed the presence of nanoparticles as the scattering process due to the colloidal suspension of nanoparticles was observed (wide band up to $550 \mathrm{~nm}$ ). The nanofluid with BAC 75\% showed it was the most stable as it had the slowest sedimentation process. Experimentally, the nanofluids with BAC were more stable than those with ODT. The evolution of the extinction coefficient values obtained from UV-vis spectra for the nanofluids prepared were followed by one week. The values decrease the first few days and after remain constant, what indicates a decrease in the number of nanoparticles in suspension that is sedimentation of nanoparticles occurs. On the other hand, the particle size was lower for the nanofluid with BAC $75 \%$ what agrees with the results obtained from UV-vis spectroscopy. To understand the role played by the surfactants in the stability of the nanofluid, theoretical calculations were performed. The interaction of the surfactants with both the base fluid and the $\mathrm{NiO}$ surface was theoretically studied. Figure 1 shows an example of the interaction of BAC surfactants with the $\mathrm{NiO}$ surface on the $\mathrm{Ni}$ as interaction site. This figure shows the size of the BAC with the benzyl ring that may present a high steric hindrance as compared to the ODT molecule that is formed by a lineal chain in the interaction with the base fluid molecule.

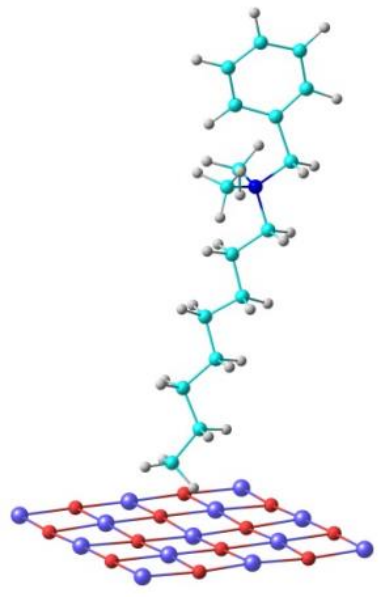

Figure 1: example of interaction of $\mathrm{BAC}$ on the $\mathrm{Ni}$ site of $\mathrm{NiO}$ surface.

For understanding the behaviour of the three nanofluids systems depending on the nature of the surfactant, molecular dynamics calculations were performed. From RDF and SDF analysis, there is 
difference in the interaction between metal oxide with the base fluid and the surfactant. For the system with $75 \% \mathrm{BAC}$ eight diphenyl oxide and one BAC molecules are around the nanoparticle. For the system with $75 \%$ of ODT there are two ODT molecules and curiously ten molecules of diphenyl oxide around the metal oxide. The SDF corresponding to this system is shown in Figure 2, where it is possible to see the 10 red lobes around the central metal oxide (blue colour) with two molecules of ODT (yellow for S and grey for the chain). The ODT molecules are oriented towar the metal oxide. The SDF for the system with a mixture of $75 \% \mathrm{BAC}$ with $75 \%$ ODT shows that there are two molecules of $\mathrm{BAC}$ next to the $\mathrm{NiO}$ with a further ODT molecule. In th 75\%BAC-75\%ODT system there are seven diphenyl oxide molecules in the inner layer around the metal oxide. Thus, when BAC is present as surfactant the number of diphenyl oxide molecules decreases as compared to those with the ODT as surfactant. So, a kind of competition between the ODT with the metal oxide and the base fluids molecules should exist. This result explains why for the system with the mixture of surfactants, the ODT molecules are far from the $\mathrm{NiO}$ nanoparticle and more molecules of BAC as surfactant are able to be around the metal oxide. Therefore, in concordance with experimental results, the theoretical results show that the nanofluid system with the BAC surfactant is the favoured one.

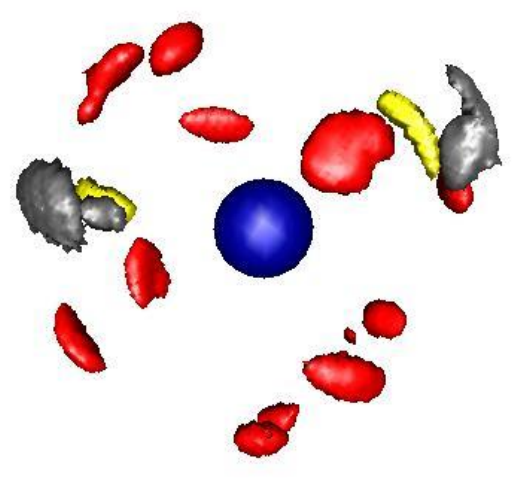

Figure 2: SDF for the NiO system with $75 \%$ of ODT.

\section{References:}

1. Singh, D., Timofeeva, E. V., Moravek, M. Cingarapu, S. R.,. Yu, W. H., Fischer, T., Mathur, S. (2014) Use of the metallic nanoparticles to improve the thermophysical properties of the organic heat transfer fluids used in concentrated solar power, Sol. Energy, 105, 468-478.

2. Kasaeian, A., Eshghi, A. T., Sameti, M. (2015) A review on the applications of nanofluids in solar energy systems. Renew Sust Energ Rev., 43, 584-598 


\title{
The role of the base fluid arrangement to enhance heat transfe of Ag-Nanofluid used for concentrating solar power plants
}

\author{
Elisa I. Martín,,${ }^{1, *}$ Antonio Sánchez-Coronilla, ${ }^{2}$ Javier Navas,${ }^{3}$ Roberto Gómez-Villarejo, ${ }^{3}$ \\ Teresa Aguilar, ${ }^{3}$ Juan Jesús Gallardo, ${ }^{3}$ Rodrigo Alcántara, ${ }^{3}$ Desireé De los \\ Santos, ${ }^{3}$ Iván Carrillo-Berdugo, ${ }^{3}$ Concha Fernández-Lorenzo ${ }^{3}$ \\ ${ }^{1}$ Seville University, Department of Chemical Engineering, Seville, Spain \\ ${ }^{2}$ Seville University, Department of Physical Chemistry, Seville, Spain \\ ${ }^{3}$ Cádiz University, Department of Physical Chemistry, Cádiz, Spain
}

Concentrating solar power is one of the most interesting alternatives to conventional energy sources nowadays. Improving the thermophysical properties of the fluids used is of particular interest for enhancing the heat transfer processes that take place. In these plants as this should result in an increase in their overall efficiency. In this sense, the use of nanofluids has been shown to be an interesting option for enhancing the thermal properties of base fluids.

In this work, nanofluids based on a eutectic mixture of diphenyl oxide and biphenyl as the base fluid were prepared varying $\mathrm{Ag}$ nanoparticle concentrations, and their physical and chemical stability, viscosity, isobaric specific heat and thermal conductivity were characterized.

According to the experimental system, molecular dynamic calculations were performed to reach a better understanding of the nanofluid system at a molecular level. The isobaric specific heat and thermal conductivity values followed the same experimental tendency, which validates the methodology proposed. An analysis of the radial distribution functions (RDFs) and spatial distribution functions (SDFs) showed the existence of a first-layer structure composed of two diphenyl oxide molecules and one biphenyl molecule placed as base of the metal nanoparticle (Figure 1).

In order to test the directionality of movement, translational distribution functions of the metal nanoparticle with respect to its average position in the system were calculated from the simulation trajectory, showing an anisotropic movement in one direction. This unexpected anisotropic traslation reflects the internal movement of the base fluid molecules belonging to the first-layer cluster. To study the rotation with regard to the metal, the orientational distribution functions of the angle formed by each oxygen of the first-layer cluster with the metal and the centre of the ring were calculated. The orientational distribution functions of the two oxygen of the first-layer cluster showed a symmetric gaussian form, a loss of intensity and an increase in amplitude up to $30^{\circ}$.

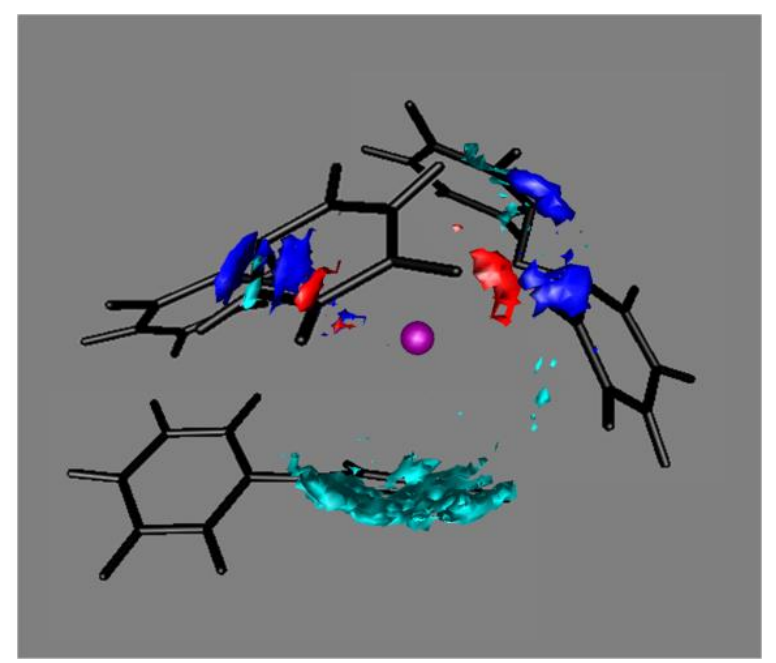

Figure 1: SDF for the Ag-nanofluid system in a radius of $4 \AA$ with the $\mathrm{Ag}$ in the centre (purple colour). The red coloured spatial distribution is assigned to $\mathrm{O}$ atoms, the blue coloured spatial distribution 
corresponds to the $\mathrm{C}$ atoms linked to the $\mathrm{O}$ atoms of the diphenyl oxide, and the sky-blue correspond to the ring $\mathrm{C}$ atoms, from the benzene rings of diphenyl oxide and biphenyl.

Regarding the torsion of the oxygen atoms with respect to the benzene ring, the orientational distribution functions of the torsion angle formed by each oxygen with the metal, the centre of the ring of biphenyl and one of their $\mathrm{C}$ atoms were calculated. In this case, the two oxygen atom of the first-layer cluster in the Ag nanofluid present a orientational distribution function with an asymmetric gaussian form. Although a loss of intensity and an increase in amplitude up to $120^{\circ}$ were observed again. Taking into account the range of rotation and torsion obtained, the two oxygen atoms of the first-layer cluster in the Ag nanofluid would describe a high fluttering movement with regard to the metal and the benzene ring. As well as, a higher range of torsion than of rotation was obtained. It indicated that rotation of the oxygens around the metal was more restricted in the direction of the benzene ring. The fact that the oxygens cannot displace the benzene ring in its rotational movement around the metal revealed the stability of this first-layer cluster.

To complete the view of the internal movement of the system, the orientational distribution functions of the angle formed by each oxygen atom of the first-layer cluster and the benzene ring with regard to the metal and the $\mathrm{x}, \mathrm{y}$ and $\mathrm{z}$ axes of the box were analysed. A clear asymmetric gaussian form was observed for the three components of the orientational distribution functions corresponding to the oxygen atom and the benzene ring, being it more noticeable for the y axis. In all cases, the amplitude of the function increased up to $90^{\circ}$. Besides the two oxygen atoms showed a different form of the functions for the $y$ axis according to the asymmetry of the orientational distribution functions of the torsion angle observed for both oxygen atoms. Thus, the marked asymmetry in the internal rotation of the $\mathrm{Ag}$ nanoparticle lead an asymmetric translational movement. So, during the trajectory the Ag nanoparticle would swing due the asymmetric internal movement of the two diphenyl oxide molecules, describing an asymmetric displacement.

The disscusion of the above results suggests that it possible to establish that the structure of first layer around of the metal nanoparticle encourages the directionality of the movement in the heart of the nanofluid and that the displacement preferably in one direction can favour effective heat transport and an increase in the thermal conductivity of the nanofluid with regard to the base fluid.

\section{References:}

1. Yoo, D. H., Hong, K. S., Yang, H. S. (2007) Study of thermal conductivity of nanofluids for the application of heat transfer fluids, Thermochim. Acta, 455,66-69.

2. Singh, D., Timofeeva, E. V., Moravek, M. Cingarapu, S. R.,. Yu, W. H., Fischer, T., Mathur, S. (2014) Use of the metallic nanoparticles to improve the thermophysical properties of the organicheat transfer fluids used in concentrated solar power, Sol. Energy, 105, 468-478. 


\title{
A Study on the Modification of Cellulose Acetate Membranes with Graphene Oxide Nanofillers for Water Treatment
}

\author{
M. Mahmoud *, A. R. Ramadan ${ }^{* *}$, and A. M. K. Esawi*** \\ *Department of Chemistry, The American University in Cairo \\ AUC Avenue, New Cairo 11835, Egypt, 3m@ aucegypt.edu \\ **Department of Chemistry, The American University in Cairo \\ AUC Avenue, New Cairo 11835, Egypt, aramadan@aucegypt.edu \\ ***Department of Mechanical Engineering, The American University in Cairo \\ AUC Avenue, New Cairo 11835, Egypt, a_esawi@aucegypt.edu
}

\begin{abstract}
In this study, cellulose acetate $(\mathrm{CA})(\mathrm{Mw}=52,000 \mathrm{Da})$ membranes containing different amounts of graphene oxide (GO) were prepared by phase inversion (PI). Acetone was used as a solvent and N, Ndimethylformamide as a non-solvent, with a ratio of 4: 1 , and deionized water as the coagulation medium. The membranes were prepared using a mixture of $19 \mathrm{wt}$. \% CA with 0.025 to $0.1 \mathrm{wt}$ \% GO. The effect of GO on membrane morphology was investigated by scanning electron microscopy (SEM), Brunauer-EmmettTeller surface analysis (BET) and contact angle measurements. Salt rejection and permeation rates were tested using a $2000 \mathrm{ppm} \mathrm{NaCl}$ solution and $5000 \mathrm{ppm} \mathrm{MgSO}_{4}$ solution at a pressure of 24 bars using a dead-end filtration cell. SEM cross sectional images showed proper dispersion of GO sheets in the polymeric matrix with no evidence of agglomerations which implies the formation of strong hydrogen bonds between CA and GO sheets. An increase in porosity of CA/GO membranes in comparison with the CA membrane was also noticed. In addition, nitrogen adsorption studies indicated that increasing GO content increased the number of macrovoids at the same final thickness of the nanocomposites believed to be due to the instantaneous solvent-non-solvent exchange as a result of the hydrophilic nature of the added GO. It was found that permeation rates increased with increasing GO. This increase in GO increased the number of hydrophilic sites in the membranes which attracted water molecules and facilitated their movement through the membrane. As for salt rejection, the membrane with $0.05 \mathrm{wt} . \% \mathrm{GO}$ showed the highest salt rejection value of $74 \%$ and $81 \%$ for $2000 \mathrm{ppm} \mathrm{NaCl}$ and $5000 \mathrm{ppm} \mathrm{MgSO}_{4}$ solutions respectively.
\end{abstract}

Keywords: cellulose acetate, graphene oxide, membranes, reverse osmosis, nanofiltration, desalination.

\section{Introduction}

Membrane desalination is a promising technology that is used in a broad range of applications. In separation applications, controlling the permeation rate of one component of a mixture through the membrane is the key property [1-3]. In order to enhance the membrane performance, different membrane preparation conditions and additives are used. Graphene oxide has been under the spotlight recently to be used as a nanofiller in polymeric materials [4]. It is considered an ideal nano-filler due to the polar oxygen-containing functionalities, hydroxyl, carbonyl, and carboxyl, on the surface of GO results in a high surface energy which increases its hydrophilicity and dispersion in organic solvents as single layer sheets [4-6]. Moreover, its ability to improve membranes hydrophilicity and alter the porosity of the membrane can significantly enhance membrane performance. On the other hand, the lack of understanding of the transport mechanism and molecular interactions of GO incorporated membranes remains a key challenge for determining the potential of these membranes in selective separation applications [7, 8]. However, the layered structure of GO with the carboxyl, hydroxyl, and epoxide groups on the basal plane and edges enables GO to have a large specific surface area which leads to a good compatibility between the host materials (polymer) and GO [9]. The current work aims to study the effect of GO incorporation on the morphology and performance of CA membranes in terms of permeability and salt retention for use in water desalination applications.

\section{Experimental \\ 2.1. Materials and methods}


GOX (ACS Material, purity $\sim 99 \%$, single layer ratio $\sim 99 \%$, thickness $\sim 0.8$ to $1.2 \mathrm{~nm}$ ) was dispersed in $N, N$ Dimethylformamide (Carlo Erba, Purity $\sim 99 \%$, density $0.949 \mathrm{~g} / \mathrm{mL}$ at $20^{\circ} \mathrm{C}$ ) by sonication for 30 minutes and added gradually to $19 \mathrm{wt} \%$ CA (average molecular weight of 50,000 Da, and $39.7 \mathrm{wt}$. \% acetyl content, Sigma Aldrich) solution in acetone (Sigma Aldrich, purity $\geq 99.8 \%$ ). The solution was stirred vigorously for 24 hours to obtain a uniform GO dispersion in the casting solution. This was followed by sonication for 15 minutes and then the solutions were allowed to stand for 10 hours to remove air bubbles. Different amounts of GOX, 0.025, 0.05, and 0.1 wt\%, were added to obtain polymer-GOX mixtures. Elcometer 4040 Automatic Film Applicator was used for the casting of the CA and CA/GO membranes on a glass substrate using a casting knife. The plate was then dipped into a coagulation bath containing distilled water for a few minutes at room temperature until complete phase inversion was ensured by solvent/non-solvent demixing. All membranes had a final thickness of $120 \pm 10 \mu \mathrm{m}$.
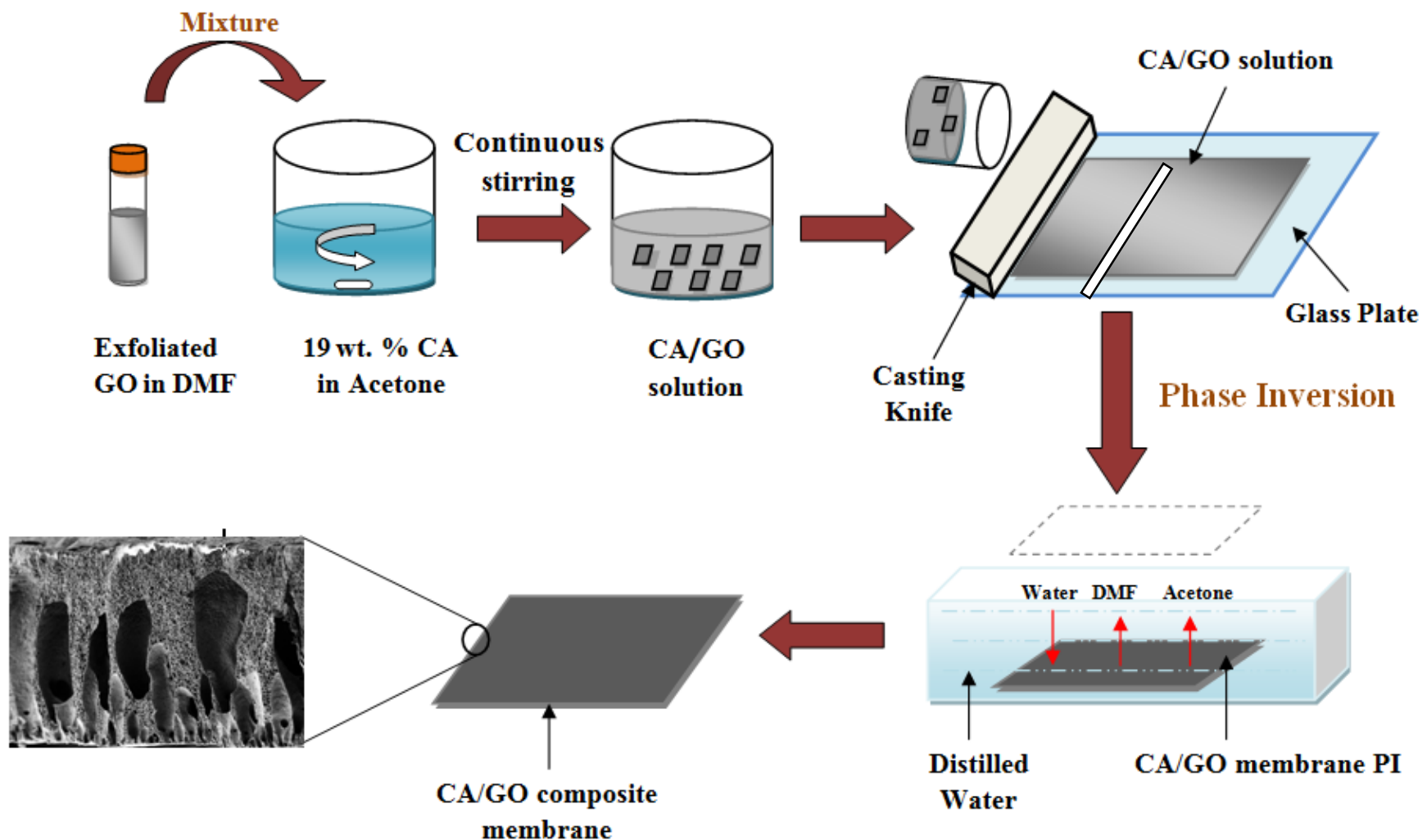

Fig. 1: Schematic diagram of the preparation process of CA/GO membrane.

Leo Supra 55 field emission scanning electron microscope (FESEM), and micrometrics ASAP 2020 instrument were used for porosity and surface area analysis of the membranes. In addition, contact angle measurements were performed to investigate the hydrophilicity of the prepared membranes using the sessile drop method by drop shape analyzer-DSA25. For water permeation and salt rejection, CA membranes were tested using a Sterlitech HP4750 Stirred Cell using a $2000 \mathrm{ppm} \mathrm{NaCl}$ and $5000 \mathrm{ppm} \mathrm{MgSO}$ (Sigma Aldrich, purity $\geq 99.5 \%$ ) feed solutions. The experiments were carried out at room temperature using $5 \mathrm{~cm}$ diameter circular membranes. $\mathrm{N}_{2}$ gas was used to apply pressures of 24 bars.

\subsection{Equations}

Equation (1) was used to calculate permeation rate, where $J$ is the permeation rate in $\mathrm{L} / \mathrm{m}^{2} \mathrm{~h}, \mathrm{~V}$ is the permeate volume, $\mathrm{A}$ is the membrane area on which pressure is applied, and $\mathrm{t}$ is the time.

$$
\mathrm{J}=\mathrm{V} /(\mathrm{At})
$$

Equation (2) was used to calculate salt rejection $R(\%)$, where $\mathrm{C}_{\mathrm{p}}$ is the salt concentration (in ppm) of the permeate and $\mathrm{C}_{\mathrm{f}}$ is the salt concentration of the feed (in ppm). 


$$
\mathrm{R}=100\left(1-\left(\mathrm{C}_{\mathrm{p}} / \mathrm{C}_{\mathrm{f}}\right)\right)
$$

To minimize the experimental errors, permeation and salt rejection for each sample was measured three times and the average values reported.

\section{Results}

\subsection{Membrane hydrophilicity}

Contact angle values presented in Table 1 show that the presence of GO in the membranes resulted in a consistent decrease in the contact angle reflecting an enhanced hydrophilic nature of the membranes with increasing GO content.

Table1: Performance of GO blended CA membranes including contact angle, BET surface area, permeation, and salt retention, with different GO contents.

\begin{tabular}{|c|c|c|c|c|c|c|}
\hline $\begin{array}{c}\text { Sample } \\
\text { Name }\end{array}$ & $\begin{array}{c}\text { Avg. } \\
\text { Contact } \\
\text { angle }\end{array}$ & $\begin{array}{c}\text { BET surface } \\
\text { area } \\
\left(\mathrm{m}^{2} / \mathrm{g}\right)\end{array}$ & $\begin{array}{c}\text { Permeation rate } \\
\left(\mathrm{L} / \mathrm{m}^{2} \mathrm{~h} . \mathrm{bar}\right) \\
2000 \mathrm{ppm} \mathrm{NaCl}\end{array}$ & $\begin{array}{c}\text { Salt } \\
\text { Retention } \\
2000 \mathrm{ppm} \\
\mathrm{NaCl}\end{array}$ & $\begin{array}{c}\text { Permeation rate } \\
\left(\mathrm{L} / \mathrm{m}^{2} \text { h.bar }\right) \\
5000 \mathrm{ppm} \\
\mathrm{MgSO}_{4}\end{array}$ & $\begin{array}{c}\text { Salt } \\
\text { Retention } \\
5000 \mathrm{ppm} \\
\mathrm{MgSO}_{4}\end{array}$ \\
\hline Blank & 75 & 1.3 & 0.010 & $55.1 \%$ & 0.008 & $60.7 \%$ \\
\hline 0.025 wt.\%GO & 69 & 1.02 & 0.017 & $57.8 \%$ & 0.009 & $73.2 \%$ \\
\hline 0.05 wt.\%GO & 67 & 2.4 & 0.020 & $73.7 \%$ & 0.01 & $81 \%$ \\
\hline 0.1 wt.\%GO & 61 & 1.4 & 0.021 & $68.1 \%$ & 0.015 & $68 \%$ \\
\hline
\end{tabular}

\subsection{Porosity}

Differential pore volume and pore surface area for all the membranes are presented in Figures 2 and 3. Pores with width larger than $50 \mathrm{~nm}$ in diameter occupied the largest volume for all membranes. On the other hand, micro-pores ,smaller than $2 \mathrm{~nm}$ in diameter, showed smaller differential pore volumes, with distinct values at pore diameter of 1.7. Although these micro-pores are very small in volume, their number must be significant to the extent that they had the highest value of total surface area of about $2.3 \mathrm{~m}^{2} / \mathrm{g}$ and $1.9 \mathrm{~m}^{2} / \mathrm{g}$ respectively for membranes with 0.05 and $0.1 \mathrm{wt} \%$ GO content. The blank membrane showed the lowest value of about $0.6 \mathrm{~m}^{2} / \mathrm{g}$. This trend can be explained by the effect of GO as a hydrophilic additive on the solvent and nonsolvent exchange rate (demixing) during phase separation.

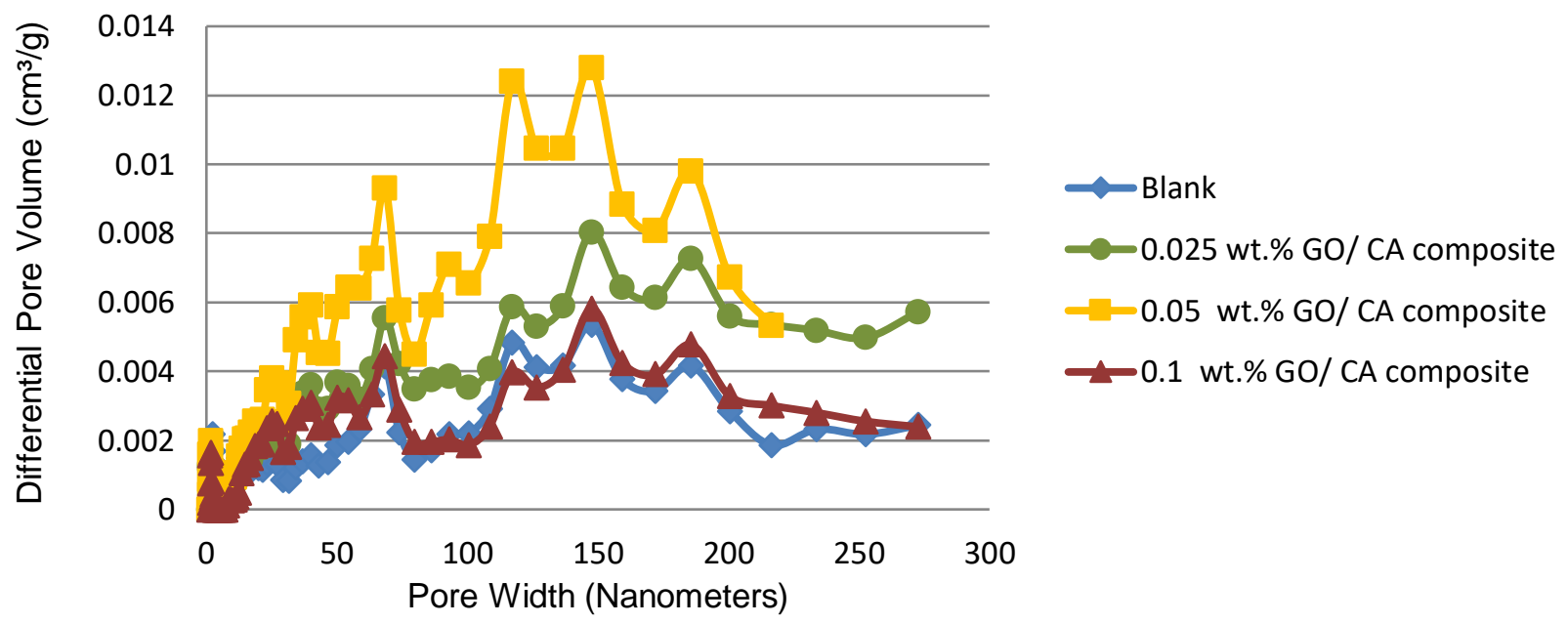

Fig. 2: Plot of the differential pore volume vs. pore width. 


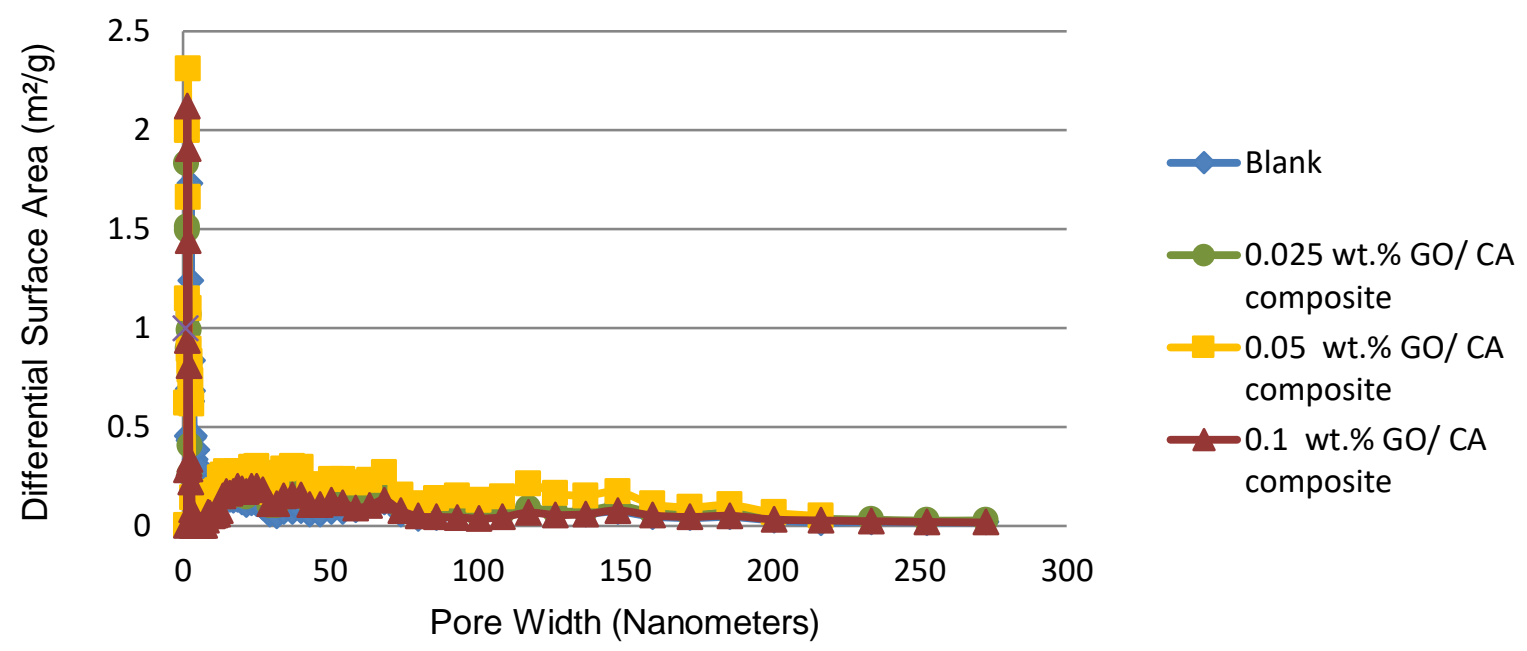

Fig. 3: Plot of the differential pore area vs. pore width.

In general, addition of GO led to an increase in membrane porosity. However, at 0.1 wt. $\%$ loading of GO, the porosity analysis showed a decrease in the micropores surface area which could be attributed to the blocking of these pores by adding high loading of $\mathrm{GO}$ which is observed as a drop in the micropores surface area. Interestingly, the BET surface area presented in Table 1 showed that the $0.05 \mathrm{wt} . \%$ GO embedded membrane has a higher surface area value than the membrane with 0.025 and $0.1 \mathrm{wt}$. \% GO, despite the fact that the permeation rates of the $0.1 \mathrm{wt}$. $\% \mathrm{GO}$ loading membranes is the highest.

\subsection{Morphology}

All membranes exhibited a structure of a skin layer followed by a much thicker finger like pore structure as detected by FESEM images. It was noticed that introducing GO in the membranes altered the membranes' structure to become more finger-like with increased porosity in comparison with those of pure CA membranes.Representative FESEM images are included in Figure 4.

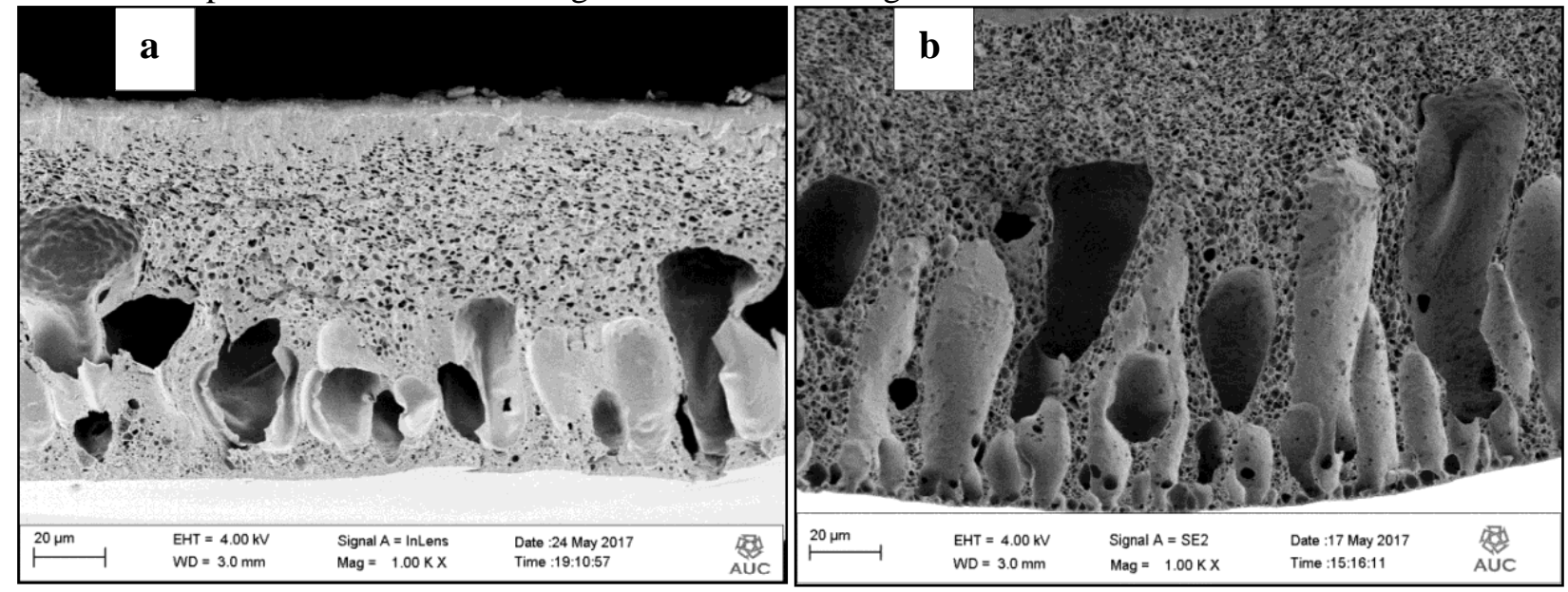



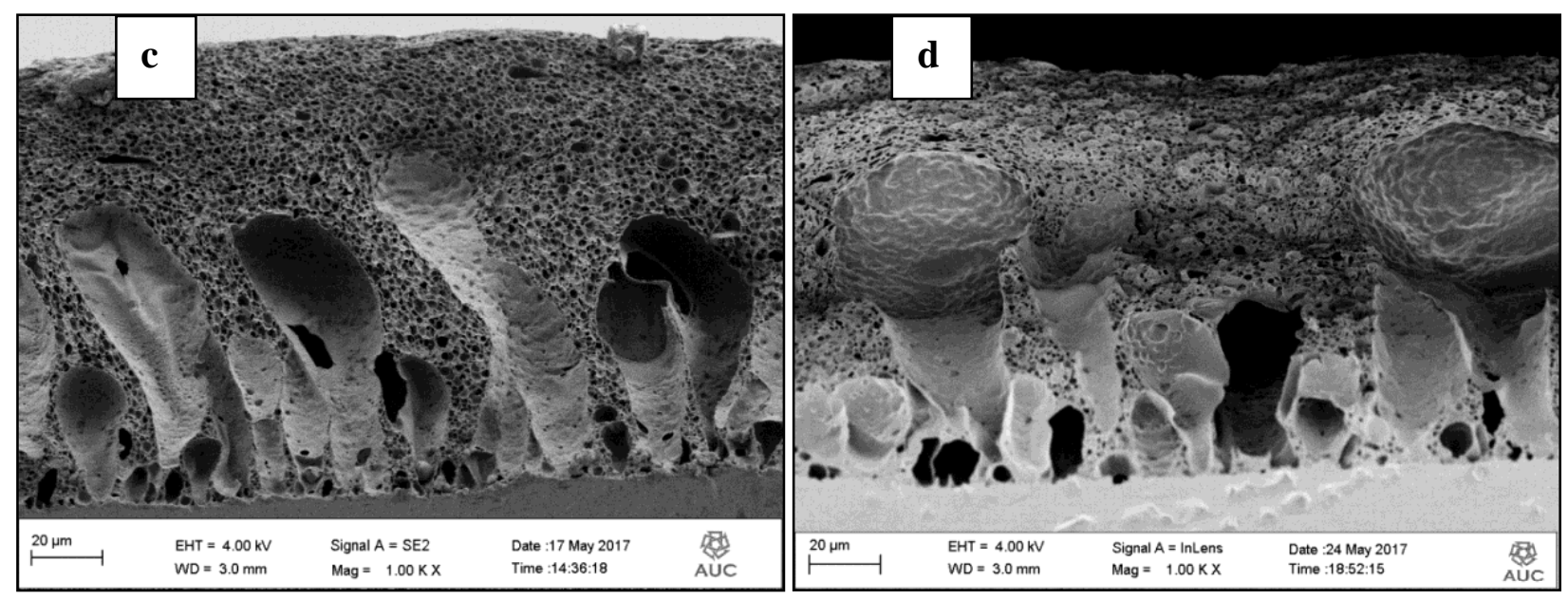

Fig 4: SEM images of pores and cross-sectional morphologies for CA/GO membranes (a) $0 \mathrm{wt} \% \mathrm{GO}$,

(b) $0.025 \mathrm{wt} . \% \mathrm{GO}$, (c) $0.05 \mathrm{wt} . \% \mathrm{GO}$, (d) $0.1 \mathrm{wt} \% \mathrm{GO}$

Based on SEM images, no GO aggregates were detected for all the samples, confirming the good dispersion of GO in the prepared cast solutions.

\subsection{Permeation and salt rejection}

Membrane water permeation and salt rejection values are presented in Table 1. Based on the obtained permeation rates, GO-blended CA membranes consistantly displayed higher permeation rates than the pure CA membrane indicating that the performance of the membranes was improved by the addition of GO, corroborated by the increase of the overall membrane porosity. With regards to the salt rejection data, the membrane with 0.05 wt. $\%$ GO demonstrated the highest salt rejection rates for both $2000 \mathrm{ppm} \mathrm{NaCl}$ and 5000 ppm $\mathrm{MgSO}_{4}$. It was interesting to observe that the increase in the salt rejection capacity of the membranes coincided with the increase in the volume of micropores in the membranes, suggesting that salt rejection rates are influenced by the pore sizes of the membranes. However, no further enhancement was detected with addition of $0.1 \mathrm{wt}$ \% which could be attributed to blocking of the micropores.

\section{Conclusions}

No agglomerations were detected for all nanocomposite CA membranes which confirmed the uniform dispersion of $\mathrm{GO}$ in the $\mathrm{CA}$ matrix.

Differential pore volumes and areas of the nanocomposite membranes showed that the membranes porosity increased with the presence of GO nano-sheets. In addition, the performance of the membranes in terms of permeation and salt retention was found to noticeably improve. The permeation rates improved with increasing GO content in the casting solutions with the $0.1 \mathrm{wt}$. \% GO incorporated membrane exhibiting the highest permeation rates. This is believed to be due to the instantaneous demixing taking place during PI after $\mathrm{GO}$ addition. Furthermore, the contact angle measurements revealed a constant decrease in contact angle upon increasing GO amount in the membranes indicating enhanced hydrophilicity of the prepared membranes. Salt rejection rates also increased with increasing GO content. The $0.05 \mathrm{wt} \% \mathrm{GO}$ membrane had the highest rejection rates believed to be due to the observed highest surface area of micropores with a volume of $1.7 \mathrm{~nm}$.

\section{References}

1. D. Pacilé, "Electronic properties and atomic structure of graphene oxide membranes," Carbon., vol. 49, no. 3, pp. 966-972, 2011.

2. D.W. Lee, "Electronic The structure of graphite oxide: investigation of its surface chemical groups," $J$. Physical Chemistry B., vol. 114, no. 17, pp. 5723-5728, 2010.

3. D. Cia, "Recent advance in functionalized graphene/polymer nanocomposites," J. Materials Chemistry., vol. 20, no. 37, pp. 7906-7915, 2010.

4. Y. Zhu, "Graphene and graphene oxide: synthesis, properties, and applications," Advanced Materials., vol. 22, no. 35, pp. 3906-3924, 2010. 
5. D. Konios, "Dispersion behaviour of graphene oxide and reduced graphene oxide," J. Colloid and Interface Science., vol. 430, pp. 108-112, 2014.

6. J.I. Paredes, “Graphene oxide dispersions in organic solvents,"Langmuir., vol. 24, no. 19, pp. 1056010564, 2008.

7. N. Wang, "Self-assembly of graphene oxide and polyelectrolyte complex nanohybrid membranes for nanofiltration and pervaporation,"J. Chemical Eng., vol.213, pp. 318-329, 2012.

8. J. Lee, "Graphene oxide nanoplatelets composite membrane with hydrophilic and antifouling properties for wastewater treatment,"J. Membrane Science., vol. 448, pp. 223-230, 2013.

9. Y. Manawi, "Can carbon-based nanomaterials revolutionize membrane fabrication for water treatment and desalination?,"Desalination., vol.391, pp. 69-88, 2016. 